\title{
A BIBLIOMETRIC ANALYSIS OF CONTEMPORARY RESEARCH REGARDING INDUSTRIAL SYMBIOSIS: A PATH TOWARDS URBAN ENVIRONMENTAL RESILIENCE
}

\author{
AKHTAR, N. ${ }^{1 *}-$ SAQIB, Z. ${ }^{2}-$ KHAN, M. I. ${ }^{1}-$ MARTIN, M. A. ${ }^{4}-$ ATIF, S. B. ${ }^{2,3}-$ ZAMAN, M. H. ${ }^{2}$ \\ ${ }^{I}$ Department of Environmental Science, International Islamic University, \\ Sector H-10, Islamabad 44000, Pakistan \\ ${ }^{2}$ GIS and Eco-Informatics Laboratory, Department of Environmental Science \\ International Islamic University, Sector H-10, Islamabad 44000, Pakistan \\ ${ }^{3}$ Department of Geography, Government College, Asghar Mall, Rawalpindi, Pakistan \\ ${ }^{4}$ IVL Swedish Environmental Research Institute, Stockholm, Sweden \\ *Corresponding author \\ e-mail:nadia@iiu.edu.pk
}

(Received 27 $7^{\text {th }}$ Sep 2018; accepted $29^{\text {th }}$ Nov 2018)

\begin{abstract}
The conceptual framework of industrial symbiosis (IS) is gaining recognition for ensuring the conservation of natural resources and resilience of socio-ecological surroundings. Significant scholastic strides have been made for explaining the conceptual paradigm of IS. The current study relied upon the Bibliometric mapping technique to decipher the contemporary orientations in the recent publications (2007-2017). The findings revealed that China, UK and the USA are the pivot for promoting research interests in the field. The loci of IS research was observed more skewed in favour of economically developed and industrialized countries. The findings of this study also acknowledge a growing propensity towards research collaboration between and among nations.
\end{abstract}

Keywords: industrial ecology, bibliometric analysis, ecological sustainability, environmental resilience, applied ecology

\section{Introduction}

Industrial symbiosis (IS) initially emerged as a branch of the industrial ecology (IE). Laybourn and Lombardi (2012) opined that the theoretical framework of IS is based on engaging diverse production activities, through an integrated network to foster ecoinnovation and long term cultural changes. This emerging branch of knowledge tries to elaborate the interconnectedness of natural environment and human society (Chertow, 2008). Therefore, an understanding regarding mutualistic relationships among organism help to re-design the process of industrial production for the sustainability of natural resources (Desrochers, 2001; Zhang et al., 2015a). The conceptual framework is gaining acceptance as a pragmatic strategy to improve efficiency in production activities (Laybourn and Lombardi, 2012). These strategies are obligatory and incumbent for ensuring the conservation of natural resources and achieving the goals of social, economic and environmental sustainability.

The paradigm of IS envisaged a framework to engage traditionally disparate industrial activities in a synchronized manner for maximizing advantages. Thus, it emphasizes on physical exchange of materials, energy, water and byproducts through integrated linkages (Chertow, 2000). The traditional modus operandi in this regard was through collaboration and exploring the synergistic possibilities that transpire through 
preconceived integrations in a limited geographic proximity. However, with the advancements in theory and practice, the scope of IS now transcends the limitations of physical proximity (Laybourn and Lombardi, 2012).

Significant scholastic efforts have been made for explaining the concept of IS, defining its boundaries, its evolution, progression, practices and different strategies adopted to implement it during the last two decades (Chertow, 2000; Mirata and Pearce, 2006; Baas, 2011; Martin and Eklund, 2011; Laybourn and Lombardi, 2012; Lombardi et al., 2012; Maclachlan, 2013; Marinos-Kouris and Mourtsiadis, 2013; Alfaro and Miller, 2014; Chertow and Park, 2016; Mauthoor, 2017). During this period, the focus of IS research witnessed a number of evolutionary transformations. Chertow and Park (2016) substantiated that the conceptual frameworks of inquiry in IS are changing in response to socio-economic and technological changes. They also perceived that such propensities are quite evident in case studies, mechanism adopted for investigations, proposals designed for investigations and modelling techniques relied upon for analysis in IS studies. In this connection, Paquin and Howard-Grenville (2013) pronounced another aspect of such evolutionary tendencies and termed it a shift from "blind dates" to "arranged marriages". Elaborating on this, they maintained that many of contemporary developments in the domain of IS research are now being facilitated by either governmental or non-governmental factors. The growing research inclinations in China towards IS are often cited to corroborate such assertions. In this country, the availability of large networks of eco-industrial parks (EIP) facilitated by the China National Demonstration Eco-Industrial Park Program are encouraging scientific publications in the field of IS. The other notable initiatives of similar objectives are National Industrial Symbiosis Program in the United Kingdom (UK) and Resource Efficiency Flagship Initiative in Europe (Laybourn and Lombardi, 2012; Paquin and Howard-Grenville, 2013).

Despite increasing importance and interest in IS, focus on recalling earlier advances in the domain appeared a less explored arena of research. Two important review papers authored by $\mathrm{Yu}$ et al. (2014) and Chertow and Park (2016) attempted to comprehensively review the orientations of research in this domain. The former researcher tried to quantitatively map and mention the noticeable strides in this domain from 1997 to 2012, while, the latter focused on the time period from 1997 to 2014. These researchers not only identified the seminal articles but also identified key themes, researchers and journals in this branch of knowledge. These researchers concluded that the field of IS stemmed from and was rooted in IE. Yu et al. (2014) identified five distinctive topical areas in the contemporary IS research i.e. wastewater treatment and management, solid waste management, energy efficiency, self-organization of IS systems, and policy making and evaluation of IS and EIP projects. Whereas, Chertow and Park (2016) sub-divided their assertions into seven categories based on the nature of study: Foundation, Performance, Mechanism, Modeling, Structure, Case Study, and Proposal.

However, both of these studies deployed bibliometric analysis technique for assessments. The Bibliometric or Scientometric mapping (Cobo et al., 2011) approach is a visual technique of informatics. This assessment approach quantitatively displays structural and dynamic aspects of scientific research proclivities for the specified temporal duration (Liu and Gui, 2016). The technique also offers replicable opportunities for quantitative estimations and visual mapping in the domain of ecology (Neff and Corley, 2009; Govindaradjou and John, 2014). These features enable to 
understand the noticeable progressions in the field of interest for systematic review and assessments. It empowers the researchers to decipher the impacts of inter-disciplinary imprints on the prevailing mode of investigations. The bibliometric analysis is also considered helpful for postulating about the emerging trajectories in research orientations ( $\mathrm{Yu}$ et al., 2014). The similar nature of methodologies have been relied upon for analyzing evolutionary developments and contemporary advances in diversified fields of knowledge (Eito-Brun and Rodríguez, 2016; Liu and Gui, 2016; Mishra et al., 2016; Atif et al., 2018).

The current research was designed to assess the contemporary trends regarding IS research by evaluating the recent publications (2012-2017). The study was designed to identify the salient features of the recent research concerning IS. Besides identifying research inclinations, the study will also attempt to provide a snapshot of key research networks and subject areas of recent publications. Thus, the present study will provide an opportunity to synchronize the efforts for environmental sustainability in the present phase of rapid population growth, urbanization and looming industrialization in the developing world.

\section{Material and methods}

The Bibliometric or Scientometric mapping technique was relied upon to decipher the contemporary research orientations in the domain of IS. This study deployed cooccurrence analysis technique to identify networks of collaborating organizations, countries, citations and co-authorships. The technique facilitates the representation of the related items with the help of networks maps through nodes and links (Liu and Gui, 2016). The size of the node is a measure of centrality and thus depicts the importance of the impacts (Wasserman and Faust, 1994). The larger nodes served as hubs in the analysis thereby depicting the significance of articles, keywords, and authors.

The technique is considered reliable ( $\mathrm{Yu}$ et al., 2014) and was deployed to analyse the spatio-temporal trends and to identify intellectual communities engaged with research concerning IS. It was subsequently relied upon for the "keyword co-occurrence analysis test" to understand the emerging developments in the domain of IS studies. Table 1 succinctly describes the nature and scope of study, data sources and the methodology implemented for data retrieval and assessments of facts.

\section{Data collection and preparation}

The current study is a meta-analysis, based upon the bibliographic information, retrieved from Scopus. The study focused on the research published between January 2012 till March 2017 against the search term "industrial symbiosis". Ostensibly, it seems that the use of a single keyword may compromise the authenticity of findings by excluding the related studies with different nomenclatures but at the same time avoiding digressions. The study carried out by Atif et al. (2018) has successfully experimented this technique. The query returned 398 records, which were further scrutinized for relevance. On this criteria, a total of 395 records (Appendix-I) were selected for further processing. The data was refined using Notepad ++ to standardize the variants used in keywords, authors, journals, organizations and country names. 
Table 1. Study approach adopted for exploring research productivity trends in IS (2012-17)

\begin{tabular}{|c|c|c|}
\hline Objectives & Questions & Indicators \\
\hline $\begin{array}{l}\text { Spatio-temporal } \\
\text { distribution of IS } \\
\text { research productivity }\end{array}$ & $\begin{array}{l}\text { How many documents are } \\
\text { published annually? } \\
\text { Which are the most productive } \\
\text { countries? } \\
\text { Key institutions involved in IS } \\
\text { research }\end{array}$ & $\begin{array}{c}\text { Number of documents published per year } \\
\text { Number of publications per country } \\
\text { Ranking of key organizations and } \\
\text { collaboration network }\end{array}$ \\
\hline $\begin{array}{l}\text { Identify most productive } \\
\text { research communities }\end{array}$ & $\begin{array}{l}\text { Who are the most productive } \\
\quad \text { authors? } \\
\text { Who are the authors that } \\
\quad \text { collaborate? } \\
\text { Who are authors that share a } \\
\text { common interest? }\end{array}$ & $\begin{array}{l}\text { Ranking of most productive authors by } \\
\text { complete count method } \\
\text { Co-authorship Analysis } \\
\text { Co-citation analysis }\end{array}$ \\
\hline Key lines of research & $\begin{array}{l}\text { Which are the key subject areas } \\
\text { under which IS research is being } \\
\text { carried out? } \\
\text { What are the key themes of IS } \\
\text { research? }\end{array}$ & $\begin{array}{c}\text { Contribution by subject based on Scopus } \\
\text { classification } \\
\text { Keyword Co-occurrences }\end{array}$ \\
\hline
\end{tabular}

\section{Data Analysis}

The data for current study was analyzed with the help of Bibexcel (Persson et al., 2009) and bibliographic networks developed in VOS Viewer (Van Eck and Waltman, 2011). Bibexcel was used due to its flexibility, ability to handle a large amount of data and its compatibility with other softwares like Excel, Pajek and VOS Viewer (Mishra et al., 2016). The network data obtained from Bibexcel was further processed in VOS Viewer to develop network maps. The findings were subsequently relied upon for depicting salient characteristics of the selected studies. The bibliometric indicators like annually published articles, country wise publication, top authors, top journals and subject categories were directly obtained from Scopus. The content analysis of the selected publications was carried out to scrutinise the causations responsible for reported orientations in contemporary research regarding IS.

\section{Results}

Since the study aims, primarily, to cover all aspects of research concerning IS for the time period from 2012 to 2017, therefore all documents, from all countries and languages available have been thoroughly scrutinized. For this purpose 395 publications, specifically related to "industrial symbiosis" were retrieved from the Scopus database for the selected time interval. These studies were carried out by the authors from 24 countries related with 20 different disciplinary areas. The data retrieved included: articles (262), other documents included conference papers (59), book chapters (28), articles in press (22), review articles (15), conference reviews (4), books (2), editorials (2) and the solitary available note. These articles were published in three languages: English (383), Chinese (10) and German (2).

In this connection, the following top five journals were observed in the forefront i.e. Journal of Cleaner Production (92), Journal of Industrial Ecology (JIE) (43), Resources, Conservation and Recycling (13), Computer Aided Chemical Engineering (11) and Shentai Xuebo Acta Ecologica Sinica (11). These five journals account for $42.7 \%$ of the total published documents. 


\section{Spatio-temporal distribution of research}

The spatio-temporal connotations of these scholastic linkages were also magnified with the help of distribution maps and statistical diagrams. The approach is considered helpful for depicting the scholastic collaborations between and among nations (Liu and Gui, 2016). The topic of IS appeared in literature around 1997, afterward, it grew exponentially with correlation coefficient $\mathrm{r}^{2}=0.88$ (Figure 1). The findings in Figure 1 also depicting the research productivity of the previous five years (2012-17) in relation to overall research published since 1997.

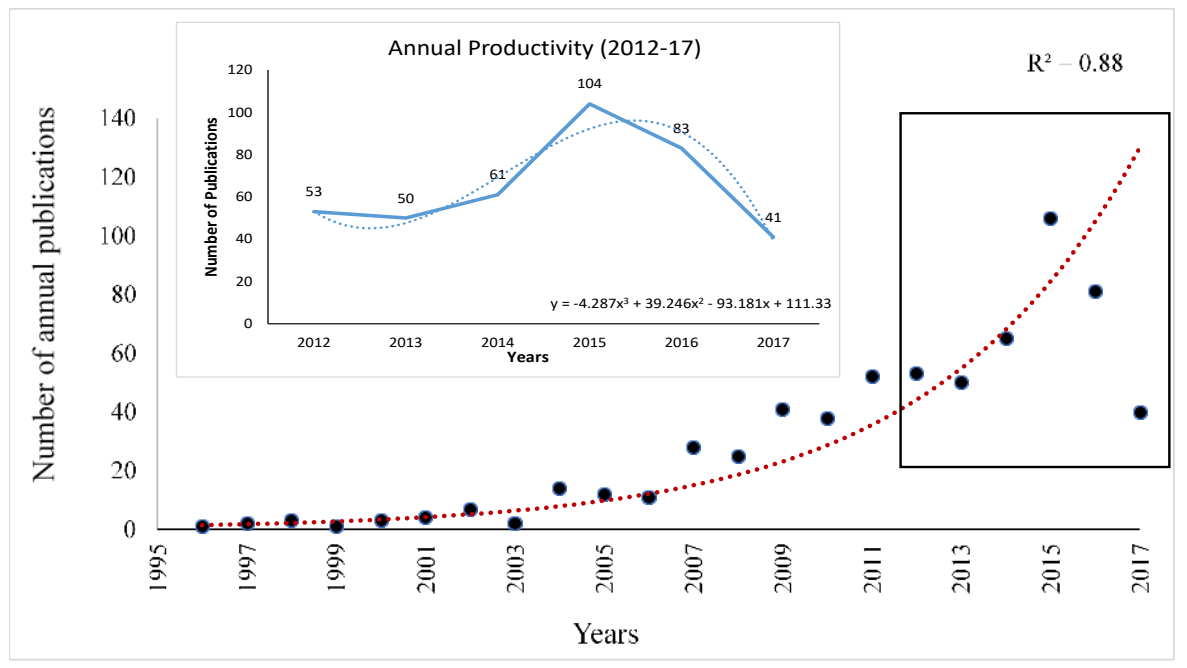

Figure 1. Annual productivity in IS research (1997-2017), while recent fluctuations encompassing the time period (2012-17) have been condensed in the inset

Spatial scope of IS has expanded to 24 countries (Figure 2) during this period and research hubs are mainly located in industrialized contextual settings (Appendix-II) such as China (85), United Kingdom (49) and United States (49). The other countries with significant contributions are Italy (34), Japan (27), Netherlands (21), Philipines (21), Australia (19), Canada (19), and France (19). The findings revealed that about 363 organizations from these countries are involved in IS research. In this connection DeLa-Salle University, Manila (22), National Institute for Environmental Studies for Japan (21), University of Surrey, United Kingdom (19), University of Tokyo, Japan (11), Tsinghua University, China (11) and the University of Nottingham, Malaysia (11) are the most prominent contributors towards IS research.

The subsequent co-occurrence frequency analysis depicted a growing tendency towards collaboration between and among different nations. In this regard, the highest scientific collaboration was observed between China and Japan in (17) cases. The participating organizations in these collaborations are the Chinese Academy of Sciences, the National Institute of Environmental Studies (NIES), Japan and Nagoya University, Japan. The researchers such as Liang Dong, Tsuyoshi Fujita affliated with NIES, Japan and Yong Geng, chief researcher in Chinese Academy of Sciences are, apparently, the most active contributors in this collaboration network. Most of these scientific research collaborations in this network were funded through various programs of Natural Science Foundation of China (11) and Ministry of Environment, Japan (8). 


\section{Research Communities}

The current study also attempted to identify the most productive authors with respect to a number of publications during the similar time period (2012-2017). The ranking is based on the complete count method. In this scheme of assessments every occurrence of the author is counted provided his name has been mentioned in the list of co-authors in a publication selected for this study. Total citations and h-index were subsequently calculated using the Bibexcel and presented in Figure 3.

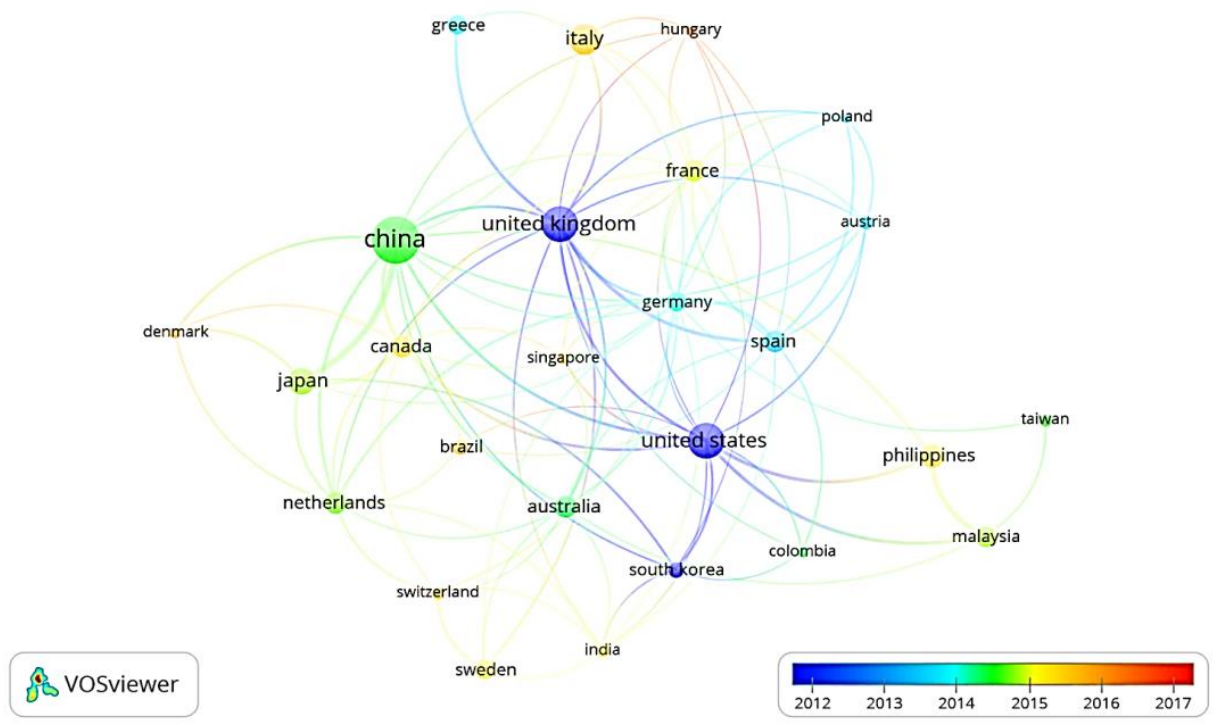

Figure 2. Geographical network of IS research productivity retrieved from corresponding author addresses (2012-17)

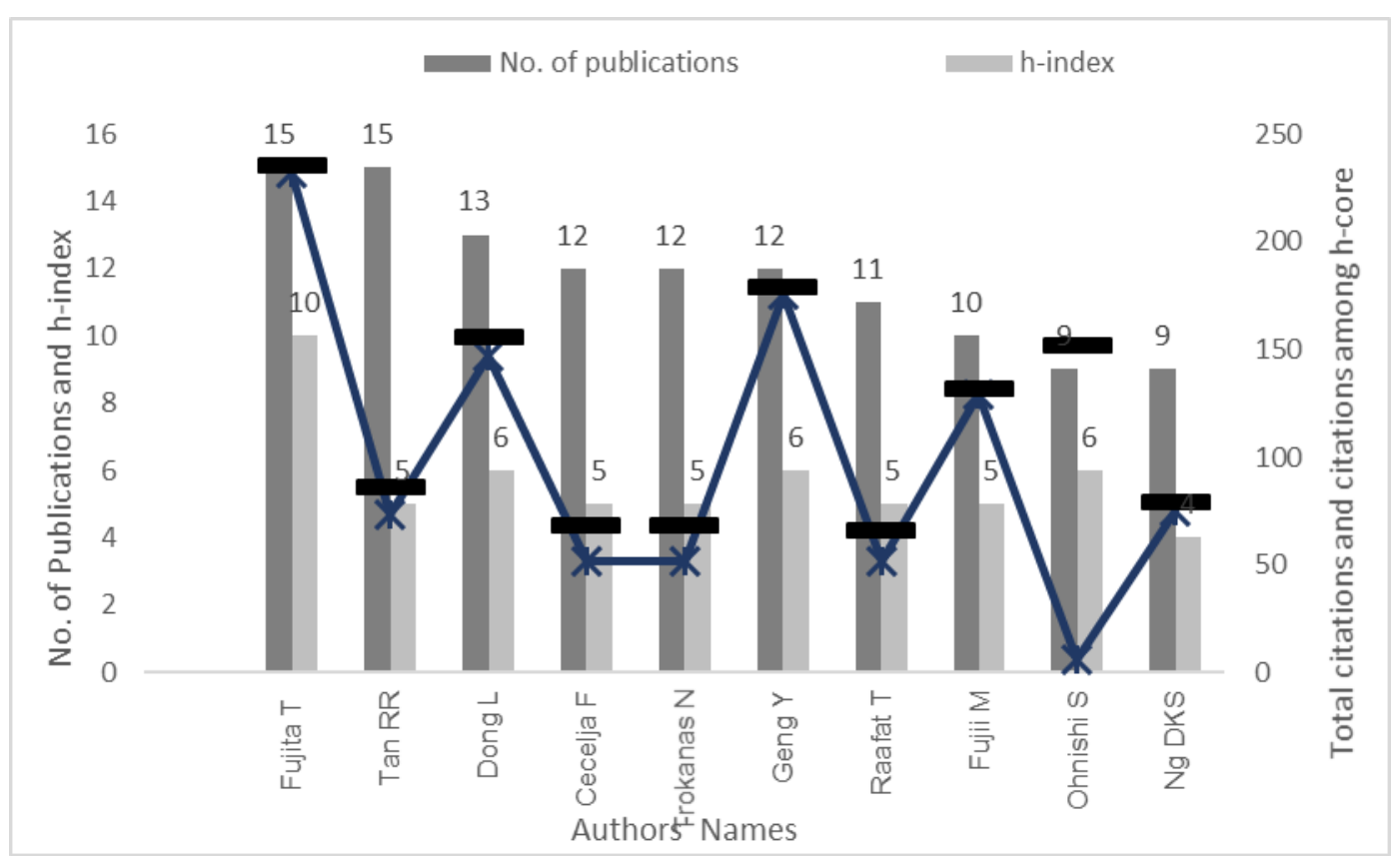

Figure 3. Ten most productive authors based on number of publications and $h$-index $(N=395)$ 
Tsuyoshi Fujita from NIES, Japan and Raymond R. Tan from De La Salle University Philippines were observed as the two most proficient and productive authors with 15 publications apiece. It is pertinent to mention that NIES, Japan (20) and De La Salle University (33) also emerged as the most productive organizations. However, scientific contributions by Tsuyoshi Fujita has received more acknowledgments in terms of citations (235), than Raymond R. Tan (86).

\section{Clusters in research collaborations}

The selected publications were analyzed to find out the scale and orientation of contemporary research collaborations. To identify the most productive collaborating authors, a co-authorship network map was developed using VOS viewer (Figure 4). The findings have been condensed in Table 2. The findings in the table explicitly convey the affiliations of authors, the focus of research and published studies ensuing from these research collaborations.

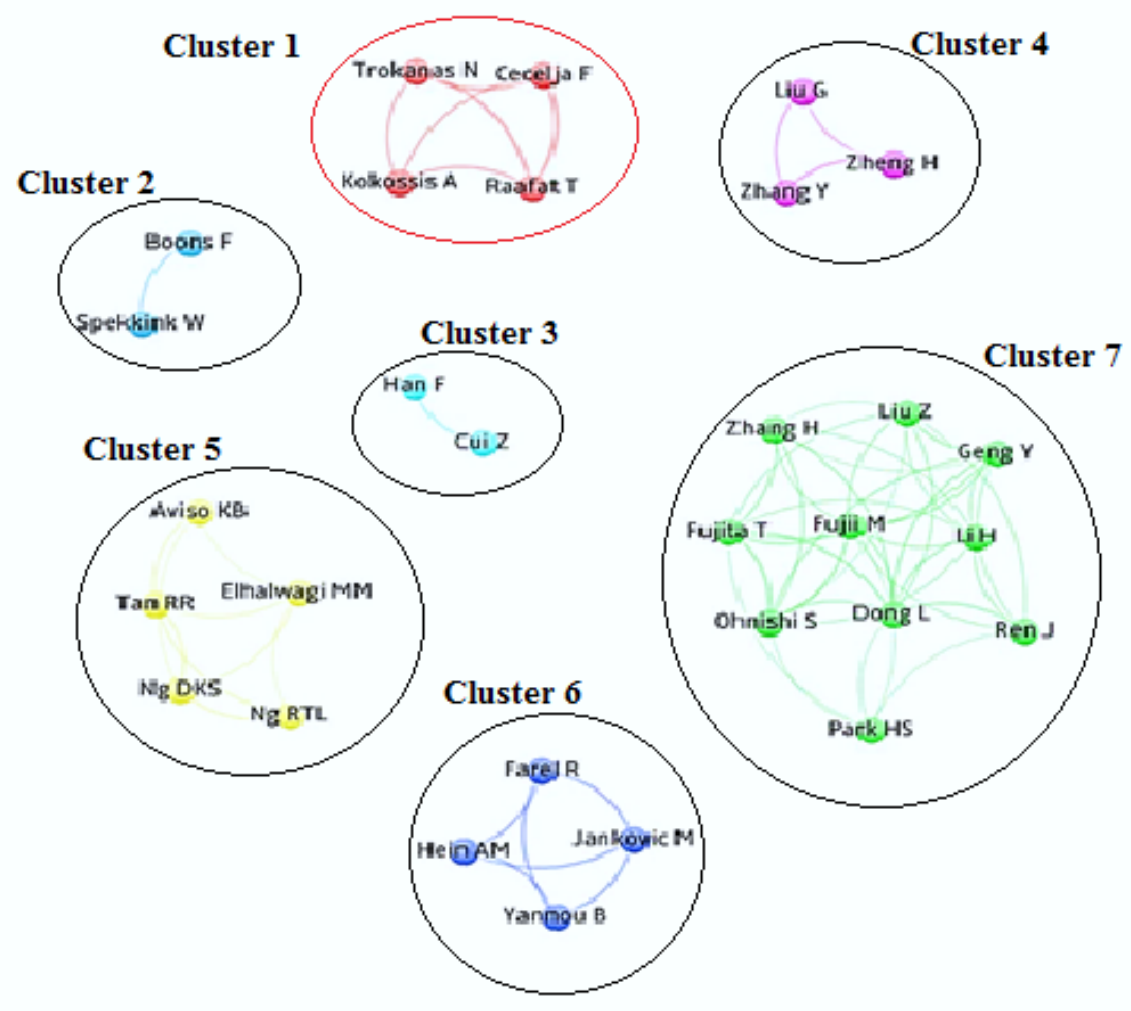

Figure 4. Co-authorship network with seven clusters working on IS

\section{Co-citation analysis}

Co-citation analysis technique is also relied upon to understand the conceptual orientations and imprints of contemporary research. The findings of current study in (Figure 5) portrayed that Marian Chertow, Yong Geng and Raymond Tan have the significant bearings and followings in the domain of IS research. 
Table 2. Co-authorship cluster classification with affiliations and research areas

\begin{tabular}{|c|c|c|c|}
\hline $\begin{array}{c}\text { Cluster } \\
\text { Number }\end{array}$ & Scholars Affiliation & Research Area & Reference Literature \\
\hline Cluster 1 & $\begin{array}{c}\text { Center for Process and Information } \\
\text { Science, Faculty of Engineering and } \\
\text { Physical Science, University of Surrey, } \\
\text { UK } \\
\text { National Technical University, Athens, } \\
\text { Greece } \\
\end{array}$ & $\begin{array}{l}\text { Semantic and } \\
\text { ontological } \\
\text { approaches for } \\
\text { IS }\end{array}$ & $\begin{array}{l}\text { (Trokanas et al., 2012; } \\
\text { Trokanas et al., 2014b; } \\
\text { Trokanas et al., 2014a; } \\
\text { Trokanas et al., 2014c; } \\
\text { Trokanas et al., 2015a; } \\
\text { Trokanas et al., 2015b) }\end{array}$ \\
\hline Cluster 2 & $\begin{array}{c}\text { Sustainable Consumption Institute, } \\
\text { University of Manchester, UK } \\
\text { University de los Andes, Bogota, } \\
\text { Columbia } \\
\end{array}$ & $\begin{array}{l}\text { IS dynamics and } \\
\text { influence of } \\
\text { different factors }\end{array}$ & $\begin{array}{c}\text { (Boons et al., 2011; Boons and } \\
\text { Spekkink, 2012; Boons et al., } \\
\text { 2014; Boons et al., 2015; Boons } \\
\text { et al., 2017) }\end{array}$ \\
\hline Cluster 3 & $\begin{array}{c}\text { School of Environmental Science and } \\
\text { Engineering, Shandong University, } \\
\text { China } \\
\end{array}$ & $\begin{array}{l}\text { IS application in } \\
\text { China }\end{array}$ & $\begin{array}{l}\text { (Yu et al., 2015b; Yu et al., } \\
\text { 2015c; Yu et al., 2015a) }\end{array}$ \\
\hline Cluster 4 & $\begin{array}{c}\text { State Key Joint Laboratory of } \\
\text { Environmental Simulation and } \\
\text { Pollution Control, School of } \\
\text { Environment, Beijing Normal } \\
\text { University, China } \\
\end{array}$ & $\begin{array}{l}\text { Network } \\
\text { analysis of IS } \\
\text { Systems }\end{array}$ & $\begin{array}{l}\text { (Zhang et al., 2013a; Zhang et } \\
\text { al., 2013b; Zhang et al., 2013c; } \\
\text { Zhang et al., 2015a; Zhang et } \\
\text { al., 2015b; Zhang et al., 2015c; } \\
\text { Zhang et al., 2016) }\end{array}$ \\
\hline Cluster 5 & $\begin{array}{l}\text { Center for Engineering andSustainable } \\
\text { Development Research, De La Salle } \\
\text { University, Manila, Philippines } \\
\text { Department of Chemical and } \\
\text { Environmental Engineering, Centre of } \\
\text { Excellence for Green Technologies, } \\
\text { University of Nottingham, Malaysia } \\
\text { Campus. Department of Chemical and } \\
\text { Biological Engineering, University of } \\
\text { Wisconsin, United States }\end{array}$ & $\begin{array}{c}\text { Fuzzy } \\
\text { programming } \\
\text { and } \\
\text { optimization } \\
\text { based IS system } \\
\text { and EIP designs }\end{array}$ & $\begin{array}{c}\text { (Ng et al., 2014a; Ng et al., } \\
\text { 2014b; Ng et al., 2014c; Tan et } \\
\text { al., 2016) }\end{array}$ \\
\hline Cluster 6 & $\begin{array}{c}\text { Laboratoire Genie Industriel, } \\
\text { CentraleSupélec, Université Paris- } \\
\text { Saclay, France, Paris-Saclay Energy } \\
\text { Efficiency (PS2E), France } \\
\end{array}$ & $\begin{array}{l}\text { EIP design } \\
\text { architecture and } \\
\text { modelling for IS }\end{array}$ & $\begin{array}{l}\text { (Hein et al., 2015a; Hein et al., } \\
\text { 2015b; Hein et al., 2016; Hein } \\
\text { et al., 2017a; Hein et al., 2017b) }\end{array}$ \\
\hline Cluster 7 & $\begin{array}{c}\text { National Institute for Environmental } \\
\text { Studies, Japan } \\
\text { National Engineering Laboratory for } \\
\text { Hydrometallurgical Cleaner } \\
\text { Production Technology, Institute of } \\
\text { Process Engineering, Chinese } \\
\text { Academy of Sciences, China. } \\
\text { Center for Social and Environmental } \\
\text { Systems Research, National Institute } \\
\text { for Environmental Studies (NIES), } \\
\text { Japan } \\
\text { Centre for Engineering Operations } \\
\text { Management, Department of } \\
\text { Technology and Innovation, University } \\
\text { of Southern Denmark. School of } \\
\text { Environmental Science and } \\
\text { Engineering, Shanghai Jiao Tong } \\
\text { University, China } \\
\text { University of Ulsan, South Korea }\end{array}$ & $\begin{array}{l}\text { Low carbon IS } \\
\text { options, } \\
\text { environmental } \\
\text { and economic } \\
\text { benefits of IS } \\
\text { for China }\end{array}$ & $\begin{array}{l}\text { (Geng et al., 2009; Dong et al., } \\
\text { 2013; Zhang et al., 2013a; } \\
\text { Zhang et al., 2013b; Zhang et } \\
\text { al., 2013c; Zhang et al., 2015a; } \\
\text { Zhang et al., 2015b; Zhang et } \\
\text { al., 2015c; Zhang et al., 2016) }\end{array}$ \\
\hline
\end{tabular}

\section{Keyword analysis}

Keyword co-occurrence maps represent the cognitive structure of a discipline. Atif et al. (2018) opined that the selection of keywords depicts the focus and orientation of the scientific research. For this purpose, the keywords from the publications were retrieved 
and processed for analysis. The findings have been illustrated in Figure 6. Only such terms as the ones recurring at least five times were mentioned. The strategy was deployed to overcome the excessive noise. Figure 6 displays an overlay visualization of the keyword co-occurrences. The size of the node reflects frequency, while, the color of the node represents the publishing time period.

The central nodes represent the keywords such as "industrial symbiosis" (277), "industrial ecology" (123), "sustainable development" (77), "industry" (76), and "ecoindustrial park" (67). Whereas, the keywords like "waste management" (43), "environmental impacts" (42) "recycling" (41), "economics" (39), "Life cycle assessment" (33) and "carbon" (25) also appeared significantly in the analysis (Figure $6)$.

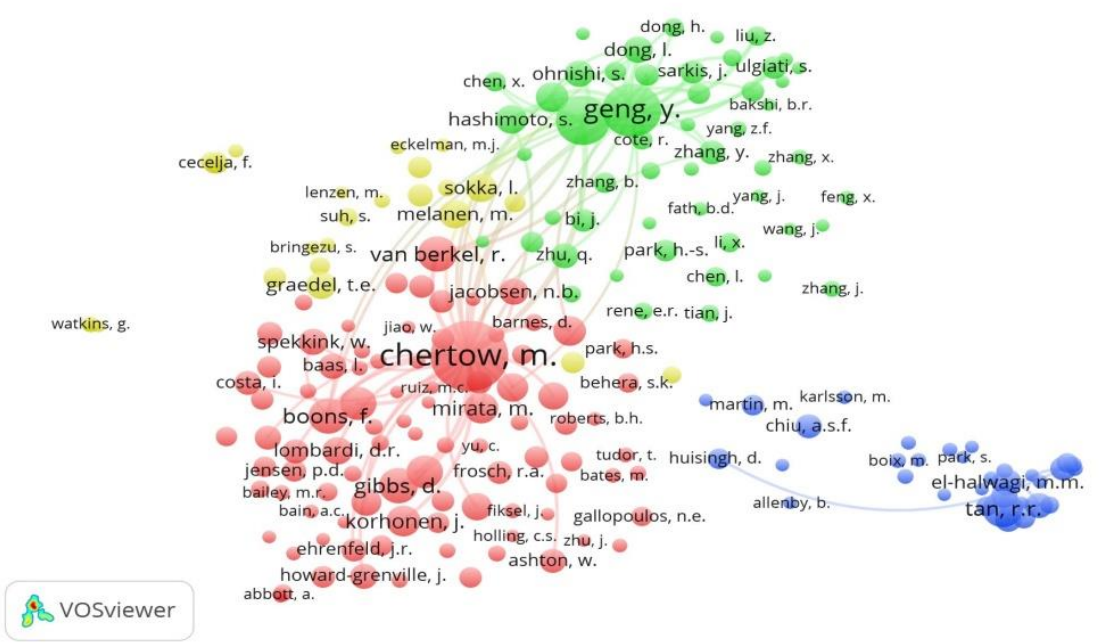

Figure 5. Co-citation network map of cited authors in documents retrieved from Scopus database

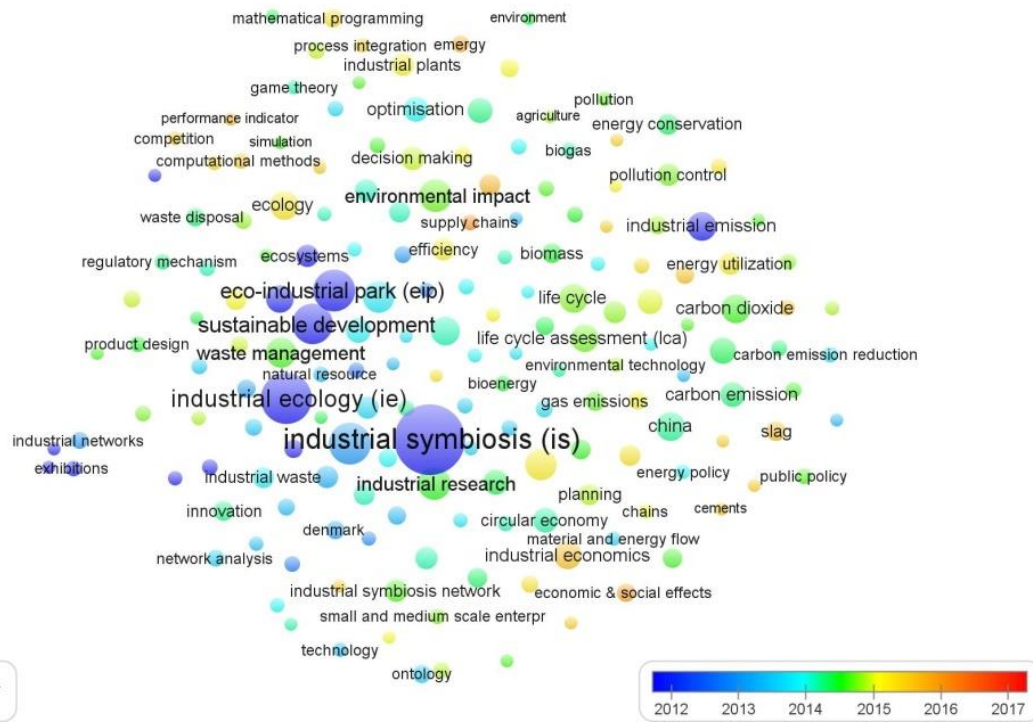

Figure 6. Keyword occurrences in IS related literature for the period 2012-17 


\section{Discussion}

The resource depletion, accelerating demands for goods and services are compelling the human conscience to strive for environmental and ecological sustainability. The researchers and policy makers are striving to postulate doable measures to achieve these goals. Chertow (2000) proclaimed that in response to these demands the research inclinations towards IS started to gain acceptability from 1997 onwards. The findings of the study in Figure 1 corroborate and substantiate these assertions. During the span of two decades (1997-2017), IS has evolved from a "signature topic" (Lifset, 2012) in IE to a more systematic, promising and advancing research discipline. As a result, the IS studies are gaining significant attention.

The findings of the study revealed that a large share (34\%) of the publications were contributed by two journals, namely Journal of Cleaner Production and Journal of Industrial Ecology (JIE); similar findings were reported by Yu et al. (2014).

The findings in the Figure 2 also pointed towards growing research collaboration between and among nations, ranging from regional to inter-continental engagements. These findings are in line with the assertions of previous investigations. In this connection, Chertow and Park (2016) and Yu et al. (2014) opined that IS is as advancing interdisciplinary field and rapidly attracting the attention of the research community. In this regard, China from Asia, UK from Europe and USA from the North America were identified as the hub and stimulator for promoting research interests in the field. Whereas, the findings of this study also portrayed that the majority of collaborations are being carried out by developed industrial economies. While the orintation towards IS research was observed in fomenting stages in the southern hemisphere. However, a growing penchant for such cross-country collaborations was also noticed, such as India (7) which is shifting from an agricultural to an industrial economic base.

The research collaborations are needed and encouraged (Iglič et al., 2017) for postulating pragmatic strategies to address the issues from divergent contextual settings. The findings based upon co-authorship analysis help to identify major research groups/collaborations in the field of IS. The findings in Figure 1 and Table 2 portrayed the emergence of seven distinctive streams of investigations as an outcome of these research collaborations. The most frequent research collaborations were observed among the researchers from China, Japan, Phillipines and UK. The most productive cluster (07) published (09) papers. This collaboration was observed among researcher from NIES, Japan, Chinese Academy of Sciences, China, University of Southern Denmark and University of Ulsan South Korea. This cluster is also the biggest in terms of number of researchers (10) engaged in collaboration.

The findings of the study (Figure 5) substantiate the previous assertions of $\mathrm{Yu}$ et al. (2014) that the scholastic contributions of Marian Chertow have more acknowledgment than any other scholar in the domain. The scholar is credited for defining the scope and sphere of this emerging research domain. In this context, Geng et al. (2009) and Tan et al. (2016) were observed as the next most influential scholars, having significant bearings on the emerging paradigm shifts in the field of IS.

The emerging dimensions of industrial symbiosis research were observed more focused on minimizing the impacts of industrial production on environmental, social and economic capitals. These initiatives are incumbent for ensuring the conservation of natural resources and the resilience of socio-ecological surroundings. 


\section{Conclusion}

The field of Industrial Symbiosis (IS) is gaining acceptance for achieving the objectives through the integration of industrial production systems and collaborative research efforts. The current study revealed growing inclinations towards IS research. The findings protrayed that field is diversifying in scope and gaining acceptance from across the globe.

The outcomes of the study also depicted that industrial economies such as China, UK and USA are spearheading the domain of IS research. The plausible explanation for the growing orientations towards IS research in China can be the premise that the industrial growth remained steadier in this region. The research paradigm in IS are mainly concieved to ensure low carbon emission through research collaboration. The policy makers and researchers in China are stressing and promoting cross-country collaborations. The research paradigms in IS are mainly conceived to ensure low carbon emissions through resarch collaborations. More focused efforts will be required in the domain of IS to achieve the ultimate goal of clean and green industrial production in the face of mounting pressures from consumer-oriented life-style changes.

Acknowledgements. The financial assistance rendered by Higher Education Commission of Pakistan (HEC, Pakistan) under National Research Program for Universities (NRPU) Project No. 4728 is acknowledged. We are also indebted to anonymous reviewers for their invaluable suggestions to improve the orientation and quality of this manuscript.

\section{REFERENCES}

[1]. Alfaro, J. , Miller, S. (2014): Applying industrial symbiosis to smallholder farms: Modeling a case study in Liberia, West Africa. - Journal of Industrial Ecology. 18: 145-154.

[2]. Atif, S., Saqib, Z., Ali, A., Zaman, M., Akhtar, N., Fatima, H., Atif, M. , Farooqi, S. (2018): Identification of Key-Trends and Evaluation of Contemporary Research Regarding Urban Ecosystem Services: A Path Towards Socio-Ecological Sustainability of Urban Areas. - APPLIED ECOLOGY AND ENVIRONMENTAL RESEARCH. 16: $3545-3581$.

[3]. Baas, L. (2011): Planning and uncovering industrial symbiosis: comparing the Rotterdam and Östergötland regions. - Business Strategy and the Environment. 20: 428-440.

[4]. Boons, F., Chertow, M., Park, J., Spekkink, W. , Shi, H. (2017): Industrial symbiosis dynamics and the problem of equivalence: Proposal for a comparative framework. Journal of Industrial Ecology. 21: 938-952.

[5]. Boons, F. , Spekkink, W. (2012): Levels of institutional capacity and actor expectations about industrial symbiosis: Evidence from the Dutch stimulation program 1999-2004. - Journal of Industrial Ecology. 16: 61-69.

[6]. Boons, F., Spekkink, W., Isenmann, R., Baas, L., Eklund, M., Brullot, S., Deutz, P., Gibbs, D., Massard, G. , Romero Arozamena, E. (2015): Comparing industrial symbiosis in Europe: Towards a conceptual framework and research methodology. - .

[7]. Boons, F., Spekkink, W. , Jiao, W. (2014): A process perspective on industrial symbiosis: Theory, methodology, and application. - Journal of Industrial Ecology. 18: 341-355. 
[8]. Boons, F., Spekkink, W. , Mouzakitis, Y. (2011): The dynamics of industrial symbiosis: a proposal for a conceptual framework based upon a comprehensive literature review. - Journal of Cleaner Production. 19: 905-911.

[9]. Chertow, M. , Park, J. (2016). Scholarship and practice in industrial symbiosis: 19892014. Taking stock of industrial ecology. Springer.

[10]. Chertow, M. R. (2000): Industrial symbiosis: literature and taxonomy. - Annual review of energy and the environment. 25: 313-337.

[11]. Chertow, M. R. (2008). Industrial ecology in a developing context. Sustainable development and environmental management. Springer.

[12]. Cobo, M. J., López-Herrera, A. G., Herrera-Viedma, E. , Herrera, F. (2011): Science mapping software tools: Review, analysis, and cooperative study among tools. Journal of the American Society for Information Science and Technology. 62: 13821402.

[13]. Desrochers, P. (2001): Cities and industrial symbiosis: Some historical perspectives and policy implications. - Journal of Industrial Ecology. 5: 29-44.

[14]. Dong, L., Fujita, T., Zhang, H., Dai, M., Fujii, M., Ohnishi, S., Geng, Y., Liu, Z. (2013): Promoting low-carbon city through industrial symbiosis: A case in China by applying HPIMO model. - Energy policy. 61: 864-873.

[15]. Eito-Brun, R. , Rodríguez, M. L. (2016): 50 years of space research in Europe: a bibliometric profile of the European Space Agency (ESA). - Scientometrics. 109: 551-576.

[16]. Geng, Y., Zhang, P., Côté, R. P. , Fujita, T. (2009): Assessment of the national eco-industrial park standard for promoting industrial symbiosis in China. - Journal of Industrial Ecology. 13: 15-26.

[17]. Govindaradjou, S. , John, D. (2014): Quantitative analysis of research trends in a leading ecological journal: bibliometric study during 2003-2012. - South African Journal of Libraries and Information Science. 80: 27-40.

[18]. Hein, A. M., Jankovic, M., Farel, R., Sam, L. I. , Yannou, B. (2015a): Modeling industrial symbiosis using design structure matrices. 17th International Dependency and Structure Modeling Conference, DMS 2015.

[19]. Hein, A. M., Jankovic, M., Farel, R., Yannou, B. (2015b): A conceptual framework for eco-industrial parks. ASME 2015 International Design Engineering Technical Conferences and Computers and Information in Engineering Conference. American Society of Mechanical Engineers, V004T005A024-V004T005A024.

[20]. Hein, A. M., Jankovic, M., Farel, R., Yannou, B. (2016): A data-and knowledge-driven methodology for generating eco-industrial park architectures. ASME 2016 International Design Engineering Technical Conferences and Computers and Information in Engineering Conference. American Society of Mechanical Engineers, V004T005A002-V004T005A002.

[21]. Hein, A. M., Jankovic, M., Feng, W., Farel, R., Yune, J. H. , Yannou, B. (2017a): Stakeholder power in industrial symbioses: A stakeholder value network approach. Journal of Cleaner Production. 148: 923-933.

[22]. Hein, A. M., Yannou, B., Jankovic, M. , Farel, R. (2017b): Towards an Automatized Generation of Rule-Based systems for architecting eco-industrial parks. International Conference on Research into Design. Springer, 691-699.

[23]. Iglič, H., Doreian, P., Kronegger, L. , Ferligoj, A. (2017): With whom do researchers collaborate and why? - Scientometrics. 112: 153-174.

[24]. Laybourn, P. , Lombardi, D. R. (2012): Industrial symbiosis in European policy. Journal of Industrial Ecology. 16: 11.

[25]. Lifset, R. (2012): Indications of Progress. - Journal of Industrial Ecology. 16: 1-1.

[26]. Liu, C. , Gui, Q. (2016): Mapping intellectual structures and dynamics of transport geography research: a scientometric overview from 1982 to 2014 . - Scientometrics. 109: $159-184$. 
[27]. Lombardi, D. R., Lyons, D., Shi, H. , Agarwal, A. (2012): Industrial symbiosis: testing the boundaries and advancing knowledge. - Journal of Industrial Ecology. 16: 2-7.

[28]. Maclachlan, I. (2013): Kwinana Industrial Area: agglomeration economies and industrial symbiosis on Western Australia's Cockburn Sound. - Australian Geographer. 44: 383-400.

[29]. Marinos-Kouris, D. , Mourtsiadis, A. (2013): INDUSTRIAL SYMBIOSIS IN GREECE: A STUDY OF SPATIAL ALLOCATION PATTERNS. - FRESENIUS ENVIRONMENTAL BULLETIN. 22: 2174-2181.

[30]. Martin, M. , Eklund, M. (2011): Improving the environmental performance of biofuels with industrial symbiosis. - Biomass and Bioenergy. 35: 1747-1755.

[31]. Mauthoor, S. (2017): Uncovering industrial symbiosis potentials in a small island developing state: The case study of Mauritius. - Journal of Cleaner Production. 147: 506-513.

[32]. Mirata, M. , Pearce, R. (2006): Industrial symbiosis in the UK. - Industrial Ecology and Spaces of Innovation (Green K and Randles S (eds)). Edward Elgar, Cheltenham, UK. 77-105.

[33]. Mishra, D., Gunasekaran, A., Papadopoulos, T. , Childe, S. J. (2016): Big Data and supply chain management: a review and bibliometric analysis. - Annals of Operations Research. 1-24.

[34]. Neff, M. W. , Corley, E. A. (2009): 35 years and 160,000 articles: A bibliometric exploration of the evolution of ecology. - Scientometrics. 80: 657-682.

[35]. Ng, R., Wan, Y., Ng, D. , Tan, R. (2014a): Stability analysis of symbiotic bioenergy parks. 17th conference on process integration, modelling and optimisation for energy saving and pollution reduction. 859-864.

[36]. Ng, R. T., Hassim, M. H., Ng, D. K., Tan, R. R. , El-Halwagi, M. M. (2014b). Multiobjective design of industrial symbiosis in palm oil industry. Computer Aided Chemical Engineering. Elsevier.

[37]. Ng, R. T., Ng, D. K., Tan, R. R. , El-Halwagi, M. M. (2014c): Disjunctive fuzzy optimisation for planning and synthesis of bioenergy-based industrial symbiosis system. - Journal of Environmental Chemical Engineering. 2: 652-664.

[38]. Paquin, R. L. , Howard-Grenville, J. (2013): Blind dates and arranged marriages: Longitudinal processes of network orchestration. - Organization Studies. 34: 16231653.

[39]. Persson, O., Danell, R. , Schneider, J. W. (2009): How to use Bibexcel for various types of bibliometric analysis. - Celebrating scholarly communication studies: A Festschrift for Olle Persson at his 60th Birthday. 5: 9-24.

[40]. Tan, R. R., Andiappan, V., Wan, Y. K., Ng, R. T. , Ng, D. K. (2016): An optimizationbased cooperative game approach for systematic allocation of costs and benefits in interplant process integration. - Chemical Engineering Research and Design. 106: 43-58.

[41]. Trokanas, N., Bussemaker, M., Velliou, E., Tokos, H. , Cecelja, F. (2015a). BiOnto: An ontology for biomass and biorefining technologies. Computer Aided Chemical Engineering. Elsevier.

[42]. Trokanas, N., Cecelja, F. , Raafat, T. (2014a): Semantic input/output matching for waste processing in industrial symbiosis. - Computers \& Chemical Engineering. 66: 259-268.

[43]. Trokanas, N., Cecelja, F. , Raafat, T. (2014b). Towards a re-usable ontology for waste processing. Computer Aided Chemical Engineering. Elsevier.

[44]. Trokanas, N., Cecelja, F. , Raafat, T. (2015b): Semantic approach for pre-assessment of environmental indicators in Industrial Symbiosis. - Journal of Cleaner Production. 96: 349-361. 
[45]. Trokanas, N., Cecelja, F., Yu, M., Raafat, T. (2014c). Optimising environmental performance of symbiotic networks using semantics. Computer Aided Chemical Engineering. Elsevier.

[46]. Trokanas, N., Raafat, T., Cecelja, F., Kokossis, A. , Yang, A. (2012). Semantic formalism for waste and processing technology classifications using ontology models. Computer aided chemical engineering. Elsevier.

[47]. Van Eck, N. J., Waltman, L. (2011): Text mining and visualization using VOSviewer. - arXiv preprint arXiv:1109.2058.

[48]. Wasserman, S. , Faust, K. (1994). Social network analysis: Methods and applications, - Cambridge university press,

[49]. Yu, C., Davis, C. , Dijkema, G. P. (2014): Understanding the evolution of industrial symbiosis research: A bibliometric and network analysis (1997-2012). - Journal of Industrial Ecology. 18: 280-293.

[50]. Yu, F., Han, F. , Cui, Z. (2015a): Assessment of life cycle environmental benefits of an industrial symbiosis cluster in China. - Environmental Science and Pollution Research. 22: 5511-5518.

[51]. Yu, F., Han, F. , Cui, Z. (2015b): Evolution of industrial symbiosis in an eco-industrial park in China. - Journal of Cleaner Production. 87: 339-347.

[52]. Yu, F., Han, F. , Cui, Z. (2015c): Reducing carbon emissions through industrial symbiosis: a case study of a large enterprise group in China. - Journal of Cleaner Production. 103: 811-818.

[53]. Zhang, H., Dong, L., Li, H.-Q., Chen, B., Tang, Q. , Fujita, T. (2013a): Investigation of the residual heat recovery and carbon emission mitigation potential in a Chinese steelmaking plant: A hybrid material/energy flow analysis case study. - Sustainable Energy Technologies and Assessments. 2: 67-80.

[54]. Zhang, H., Dong, L., Li, H., Fujita, T., Ohnishi, S. , Tang, Q. (2013b): Analysis of lowcarbon industrial symbiosis technology for carbon mitigation in a Chinese iron/steel industrial park: a case study with carbon flow analysis. - Energy policy. 61: 14001411.

[55]. Zhang, Y., Zheng, H., Chen, B., Su, M. , Liu, G. (2015a): A review of industrial symbiosis research: theory and methodology. - Frontiers of Earth Science. 9: 91-104.

[56]. Zhang, Y., Zheng, H., Chen, B. , Yang, N. (2013c): Social network analysis and network connectedness analysis for industrial symbiotic systems: model development and case study. - Frontiers of Earth Science. 7: 169-181.

[57]. Zhang, Y., Zheng, H. , Fath, B. D. (2015b): Ecological network analysis of an industrial symbiosis system: a case study of the Shandong Lubei eco-industrial park. Ecological Modelling. 306: 174-184.

[58]. Zhang, Y., Zheng, H., Shi, H., Yu, X., Liu, G., Su, M., Li, Y. , Chai, Y. (2016): Network analysis of eight industrial symbiosis systems. - Frontiers of Earth Science. 10: 352-365.

[59]. Zhang, Y., Zheng, H., Yang, Z., Liu, G. , Su, M. (2015c): Analysis of the industrial metabolic processes for sulfur in the Lubei (Shandong Province, China) eco-industrial park. - Journal of Cleaner Production. 96: 126-138. 


\section{APPENDIX}

Appendix-I. 395 selected and reviewed articles

\begin{tabular}{|c|c|c|c|c|c|c|c|c|}
\hline Author Name(s) & Title & Source Title & Year & Vol. & Issue & P. Start & P. End & Doi \\
\hline $\begin{array}{c}\text { Zhou X., Zhang } \\
\text { H. }\end{array}$ & $\begin{array}{c}\text { Research on industrial } \\
\text { symbiosis mode logistics } \\
\text { industrial cluster in Shenyang } \\
\text { Economic Zone }\end{array}$ & $\begin{array}{c}\text { Proceeding of } 2012 \\
\text { International } \\
\text { Conference on } \\
\text { Information } \\
\text { Management, } \\
\text { Innovation } \\
\text { Management and } \\
\text { Industrial Engineering, } \\
\text { ICIII } 2012 \\
\end{array}$ & 2012 & 2 & & 489 & 492 & \\
\hline $\begin{array}{l}\text { Romero D., } \\
\text { Molina A. }\end{array}$ & $\begin{array}{l}\text { Green virtual enterprise } \\
\text { breeding environments: A } \\
\text { sustainable industrial } \\
\text { development model for a } \\
\text { circular economy } \\
\end{array}$ & $\begin{array}{l}\text { IFIP Advances in } \\
\text { Information and } \\
\text { Communication } \\
\text { Technology }\end{array}$ & 2012 & $\begin{array}{c}380 \\
\text { AICT }\end{array}$ & & 427 & 436 & 10.1007/978-3-642-32775-9_43 \\
\hline $\begin{array}{l}\text { Kopacek B., } \\
\text { Schadlbauer S. }\end{array}$ & Introduction to zero WIN & $\begin{array}{l}\text { Electronics Goes Green } \\
\text { 2012+, ECG } 2012 \text { - } \\
\text { Joint International } \\
\text { Conference and } \\
\text { Exhibition, Proceedings }\end{array}$ & 2012 & & & & & \\
\hline $\begin{array}{l}\text { Schadlbauer S., } \\
\text { Kopacek B., } \\
\text { Gallo M., Arnaiz } \\
\text { S. }\end{array}$ & $\begin{array}{l}\text { The ZeroWIN production } \\
\text { model }\end{array}$ & $\begin{array}{c}\text { Electronics Goes Green } \\
\text { 2012+, ECG } 2012 \text { - } \\
\text { Joint International } \\
\text { Conference and } \\
\text { Exhibition, Proceedings }\end{array}$ & 2012 & & & & & \\
\hline $\begin{array}{c}\text { Arranz P., } \\
\text { Tarragó J., Vallvé } \\
\text { X., Marwede M., } \\
\text { Den Boer E., } \\
\text { Rothe M., Wüst } \\
\text { F., Middendorf } \\
\text { A., Cocciantelli } \\
\text { J.-M., Lippert M. }\end{array}$ & $\begin{array}{l}\text { Practical demonstrator 'Design } \\
\text { for recycling photovoltaic } \\
\text { system' }\end{array}$ & $\begin{array}{c}\text { Electronics Goes Green } \\
\text { 2012+, ECG } 2012 \text { - } \\
\text { Joint International } \\
\text { Conference and } \\
\text { Exhibition, Proceedings }\end{array}$ & 2012 & & & & & \\
\hline
\end{tabular}

APPLIED ECOLOGY AND ENVIRONMENTAL RESEARCH 17(1):1159-1221.

http://www.aloki.hu • ISSN 15891623 (Print) • ISSN 17850037 (Online)

DOI: http://dx.doi.org/10.15666/aeer/1701_11591221

(c) 2019, ALÖKI Kft., Budapest, Hungary 


\begin{tabular}{|c|c|c|c|c|c|c|c|c|}
\hline Author Name(s) & Title & Source Title & Year & Vol. & Issue & P. Start & P. End & Doi \\
\hline $\begin{array}{l}\text { Marwede M., } \\
\text { Schischke K., } \\
\text { Arranz P., Hickey } \\
\text { S., Fitzpatrick C., } \\
\text { Ospina J., Yang } \\
\text { M., Nissen N.F., } \\
\text { Lang K.-D. }\end{array}$ & $\begin{array}{l}\text { Methodology to identify design } \\
\text { for recycling measures for } \\
\text { high-tech sectors }\end{array}$ & $\begin{array}{c}\text { Electronics Goes Green } \\
2012+, \text { ECG 2012 - } \\
\text { Joint International } \\
\text { Conference and } \\
\text { Exhibition, Proceedings }\end{array}$ & 2012 & & & & & \\
\hline $\begin{array}{l}\text { Nesbit S., Stano } \\
\text { J., Atwater J.W., } \\
\text { Casavant T. }\end{array}$ & $\begin{array}{l}\text { Cascading water: Combining } \\
\text { GIS and system analysis to } \\
\text { maximize water reuse }\end{array}$ & $\begin{array}{l}\text { Canadian Journal of } \\
\text { Civil Engineering }\end{array}$ & 2012 & 39 & 12 & 1321 & 1327 & $10.1139 /$ cjce-2012-0251 \\
\hline $\begin{array}{l}\text { Den Boer E., } \\
\text { Williams I., } \\
\text { Fitzpatrick C., } \\
\text { Arranz P., } \\
\text { Dietrich J., Kent } \\
\text { A., Tischer A., } \\
\text { Durao V., Perthes } \\
\text { H., Peagam R., } \\
\text { Kopacek B. }\end{array}$ & $\begin{array}{l}\text { Bringing all industrial networks } \\
\text { together and next steps }\end{array}$ & $\begin{array}{c}\text { Electronics Goes Green } \\
\text { 2012+, ECG 2012 - } \\
\text { Joint International } \\
\text { Conference and } \\
\text { Exhibition, Proceedings }\end{array}$ & 2012 & & & & & \\
\hline Li G. & $\begin{array}{l}\text { A paradigm of constructing } \\
\text { industrial symbiosis and } \\
\text { coupling in China's county- } \\
\text { region economic sustainable } \\
\text { development }\end{array}$ & $\begin{array}{c}\text { Green Technologies } \\
\text { and Business Practices: } \\
\text { An IT Approach }\end{array}$ & 2012 & & & 1 & 14 & 10.4018/978-1-4666-1972-2.ch001 \\
\hline $\begin{array}{l}\text { Watts C., Binder } \\
\text { C.R. }\end{array}$ & $\begin{array}{l}\text { Simulating shocks with the } \\
\text { hypercycles model of economic } \\
\text { production }\end{array}$ & $\begin{array}{c}\text { iEMSs } 2012 \text { - } \\
\text { Managing Resources of } \\
\text { a Limited Planet: } \\
\text { Proceedings of the 6th } \\
\text { Biennial Meeting of the } \\
\text { International } \\
\text { Environmental } \\
\text { Modelling and Software } \\
\text { Society }\end{array}$ & 2012 & & & 2651 & 2659 & \\
\hline Diwekar U. & $\begin{array}{l}\text { Green engineering and } \\
\text { sustainability: A systems } \\
\text { analysis perspective }\end{array}$ & $\begin{array}{c}\text { Sustainability: Multi- } \\
\text { Disciplinary } \\
\text { Perspectives } \\
\end{array}$ & 2012 & & & 273 & 309 & $10.2174 / 978160805103811201010273$ \\
\hline
\end{tabular}

APPLIED ECOLOGY AND ENVIRONMENTAL RESEARCH 17(1):1159-1221.

http://www.aloki.hu • ISSN 15891623 (Print) • ISSN 17850037 (Online)

DOI: http://dx.doi.org/10.15666/aeer/1701_11591221

(c) 2019, ALÖKI Kft., Budapest, Hungary 


\begin{tabular}{|c|c|c|c|c|c|c|c|c|}
\hline Author Name(s) & Title & Source Title & Year & Vol. & Issue & P. Start & P. End & Doi \\
\hline $\begin{array}{l}\text { Karkanias C., } \\
\text { Karagiannidis A., } \\
\text { Antonopoulos } \\
\text { I.S., Samaras P. }\end{array}$ & $\begin{array}{l}\text { Adopting rational waste } \\
\text { management schemes: The } \\
\text { case of Preveza municipality }\end{array}$ & $\begin{array}{l}\text { Economics and Policy } \\
\text { of Energy and the } \\
\text { Environment }\end{array}$ & 2012 & & 3 & 65 & 79 & \\
\hline $\begin{array}{l}\text { Hiete M., Ludwig } \\
\text { J., Schultmann F. }\end{array}$ & $\begin{array}{c}\text { Intercompany Energy } \\
\text { Integration: Adaptation of } \\
\text { Thermal Pinch Analysis and } \\
\text { Allocation of Savings }\end{array}$ & $\begin{array}{l}\text { Journal of Industrial } \\
\text { Ecology }\end{array}$ & 2012 & 16 & 5 & 689 & 698 & $10.1111 / \mathrm{j} .1530-9290.2012 .00462 . x$ \\
\hline $\begin{array}{l}\text { Wells P., Zapata } \\
\text { C. }\end{array}$ & $\begin{array}{l}\text { Renewable Eco-industrial } \\
\text { Development: A New Frontier } \\
\text { for Industrial Ecology? }\end{array}$ & $\begin{array}{l}\text { Journal of Industrial } \\
\text { Ecology }\end{array}$ & 2012 & 16 & 5 & 665 & 668 & 10.1111/j.1530-9290.2012.00487.x \\
\hline $\begin{array}{l}\text { Chopra S.S., } \\
\text { Khanna V. }\end{array}$ & $\begin{array}{l}\text { Toward a network perspective } \\
\text { for understanding resilience } \\
\text { and sustainability in industrial } \\
\text { symbiotic networks }\end{array}$ & $\begin{array}{l}\text { IEEE International } \\
\text { Symposium on } \\
\text { Sustainable Systems } \\
\text { and Technology }\end{array}$ & 2012 & & & & & 10.1109/ISSST.2012.6227987 \\
\hline Lin K.-N. & $\begin{array}{l}\text { Cradle to cradle at CSC: } \\
\text { Through integrated recycling } \\
\text { system and industrial symbiosis }\end{array}$ & $\begin{array}{c}\text { AISTech - Iron and } \\
\text { Steel Technology } \\
\text { Conference Proceedings }\end{array}$ & 2012 & & & 217 & 224 & \\
\hline $\begin{array}{l}\text { Usón S., Valero } \\
\text { A., Agudelo A. }\end{array}$ & $\begin{array}{c}\text { Thermoeconomics and } \\
\text { Industrial Symbiosis. Effect of } \\
\text { by-product integration in cost } \\
\text { assessment }\end{array}$ & Energy & 2012 & 45 & 1 & 43 & 51 & 10.1016/j.energy.2012.04.016 \\
\hline $\begin{array}{l}\text { Ohnishi S., Fujita } \\
\text { T., Chen X., Fujii } \\
\text { M. }\end{array}$ & $\begin{array}{c}\text { Econometric analysis of the } \\
\text { performance of recycling } \\
\text { projects in Japanese Eco- } \\
\text { Towns }\end{array}$ & $\begin{array}{l}\text { Journal of Cleaner } \\
\text { Production }\end{array}$ & 2012 & 33 & & 217 & 225 & 10.1016/j.jclepro.2012.03.027 \\
\hline $\begin{array}{l}\text { Clark J.H., } \\
\text { Pfaltzgraff L. }\end{array}$ & $\begin{array}{l}\text { Industrial symbiosis using } \\
\text { green chemistry }\end{array}$ & $\begin{array}{l}\text { Technical Proceedings } \\
\text { of the } 2012 \text { NSTI } \\
\text { Nanotechnology } \\
\text { Conference and Expo, } \\
\text { NSTI-Nanotech } 2012\end{array}$ & 2012 & & & 706 & 707 & \\
\hline $\begin{array}{l}\text { Raafat T., } \\
\text { Trokanas N., } \\
\text { Cecelja F., } \\
\text { Kokossis A., } \\
\text { Yang A. } \\
\end{array}$ & $\begin{array}{l}\text { Semantically-enabled } \\
\text { Formalisation to Support and } \\
\text { Automate the Application of } \\
\text { Industrial Symbiosis }\end{array}$ & $\begin{array}{l}\text { Computer Aided } \\
\text { Chemical Engineering }\end{array}$ & 2012 & 31 & & 1055 & 1059 & 10.1016/B978-0-444-59506-5.50042-0 \\
\hline
\end{tabular}




\begin{tabular}{|c|c|c|c|c|c|c|c|c|}
\hline Author Name(s) & Title & Source Title & Year & Vol. & Issue & P. Start & P. End & Doi \\
\hline $\begin{array}{l}\text { Shi L., Liu G., } \\
\text { Guo S. }\end{array}$ & $\begin{array}{l}\text { International comparison and } \\
\text { policy recommendation on the } \\
\text { development model of } \\
\text { industrial symbiosis in China }\end{array}$ & $\begin{array}{c}\text { Shengtai Xuebao/ Acta } \\
\text { Ecologica Sinica }\end{array}$ & 2012 & 32 & 12 & 3950 & 3957 & 10.5846/stxb201111131724 \\
\hline $\begin{array}{l}\text { Shi X., Yang J., } \\
\text { Wang R., Zhao L. }\end{array}$ & $\begin{array}{l}\text { An approach for analyzing } \\
\text { resources metabolism of } \\
\text { industrial ecosystems }\end{array}$ & $\begin{array}{c}\text { Shengtai Xuebao/ Acta } \\
\text { Ecologica Sinica }\end{array}$ & 2012 & 32 & 7 & 2012 & 2024 & $10.5846 / \mathrm{stxb} 201104180505$ \\
\hline $\begin{array}{l}\text { Meneghetti A., } \\
\text { Nardin G. }\end{array}$ & $\begin{array}{c}\text { Enabling industrial symbiosis } \\
\text { by a facilities management } \\
\text { optimization approach }\end{array}$ & $\begin{array}{l}\text { Journal of Cleaner } \\
\text { Production }\end{array}$ & 2012 & 35 & & 263 & 273 & 10.1016/j.jclepro.2012.06.002 \\
\hline $\begin{array}{c}\text { Raafat T., Cecelja } \\
\text { F., Yang A., } \\
\text { Trokanas N. } \\
\end{array}$ & $\begin{array}{l}\text { Semantic support for industrial } \\
\text { symbiosis process }\end{array}$ & $\begin{array}{c}\text { Computer Aided } \\
\text { Chemical Engineering }\end{array}$ & 2012 & 30 & & 452 & 456 & 10.1016/B978-0-444-59519-5.50091-5 \\
\hline $\begin{array}{c}\text { Trokanas N., } \\
\text { Raafat T., Cecelja } \\
\text { F., Kokossis A., } \\
\text { Yang A. }\end{array}$ & $\begin{array}{l}\text { Semantic Formalism for Waste } \\
\text { and Processing Technology } \\
\text { Classifications Using Ontology } \\
\text { Models }\end{array}$ & $\begin{array}{c}\text { Computer Aided } \\
\text { Chemical Engineering }\end{array}$ & 2012 & 30 & & 167 & 171 & 10.1016/B978-0-444-59519-5.50034-4 \\
\hline $\begin{array}{l}\text { Behera S.K., Kim } \\
\text { J.-H., Lee S.-Y., } \\
\text { Suh S., Park H.- } \\
\text { S. }\end{array}$ & $\begin{array}{c}\text { Evolution of 'designed' } \\
\text { industrial symbiosis networks } \\
\text { in the Ulsan Eco-industrial } \\
\text { Park: 'Research and } \\
\text { development into business' as } \\
\text { the enabling framework }\end{array}$ & $\begin{array}{l}\text { Journal of Cleaner } \\
\text { Production }\end{array}$ & 2012 & $29-30$ & & 103 & 112 & 10.1016/j.jclepro.2012.02.009 \\
\hline $\begin{array}{l}\text { Liu L., Zhang B., } \\
\text { Bi J., Wei Q., He } \\
\text { P. }\end{array}$ & $\begin{array}{c}\text { The greenhouse gas mitigation } \\
\text { of industrial parks in China: A } \\
\text { case study of Suzhou Industrial } \\
\text { Park }\end{array}$ & Energy Policy & 2012 & 46 & & 301 & 307 & 10.1016/j.enpol.2012.03.064 \\
\hline $\begin{array}{l}\text { Geißen S.-U., } \\
\text { Bennemann H., } \\
\text { Horn H., Krull } \\
\text { R., Neumann S. }\end{array}$ & $\begin{array}{l}\text { Industrial wastewater treatment } \\
\text { and recycling - Potentials and } \\
\text { prospects } \\
\text { [Industrieabwasserbehandlung } \\
\text { und -recycling - Potenziale und } \\
\text { Perspektiven] } \\
\end{array}$ & $\begin{array}{c}\text { Chemie-Ingenieur- } \\
\text { Technik }\end{array}$ & 2012 & 84 & 7 & 1005 & 1017 & 10.1002/cite.201200006 \\
\hline $\begin{array}{l}\text { Andrade L.C., } \\
\text { Míguez C.G., } \\
\text { Gómez M.C.T., } \\
\text { Bugallo P.M.B. }\end{array}$ & $\begin{array}{l}\text { Management strategy for } \\
\text { hazardous waste from atomised } \\
\text { SME: Application to the } \\
\text { printing industry }\end{array}$ & $\begin{array}{l}\text { Journal of Cleaner } \\
\text { Production }\end{array}$ & 2012 & 35 & & 214 & 229 & 10.1016/j.jclepro.2012.05.014 \\
\hline
\end{tabular}




\begin{tabular}{|c|c|c|c|c|c|c|c|c|}
\hline Author Name(s) & Title & Source Title & Year & Vol. & Issue & P. Start & P. End & Doi \\
\hline $\begin{array}{l}\text { Geng D., Li J., } \\
\text { Liu J., Song X. }\end{array}$ & $\begin{array}{l}\text { System analysis of circular } \\
\text { economy development in coal } \\
\text { mining area }\end{array}$ & $\begin{array}{l}\text { Advanced Materials } \\
\text { Research }\end{array}$ & 2012 & $\begin{array}{l}524- \\
527\end{array}$ & & 2735 & 2740 & $\begin{array}{c}\text { 10.4028/www.scientific.net/AMR.524- } \\
527.2735\end{array}$ \\
\hline $\begin{array}{l}\text { Blengini G.A., } \\
\text { Busto M., Fantoni } \\
\text { M., Fino D. }\end{array}$ & $\begin{array}{l}\text { Eco-efficient waste glass } \\
\text { recycling: Integrated waste } \\
\text { management and green product } \\
\text { development through LCA }\end{array}$ & Waste Management & 2012 & 32 & 5 & 1000 & 1008 & 10.1016/j.wasman.2011.10.018 \\
\hline $\begin{array}{l}\text { Golev A., Corder } \\
\text { G.D. }\end{array}$ & $\begin{array}{l}\text { Developing a classification } \\
\text { system for regional resource } \\
\text { synergies }\end{array}$ & Minerals Engineering & 2012 & 29 & & 58 & 64 & 10.1016/j.mineng.2011.10.018 \\
\hline $\begin{array}{l}\text { Geng Y., Fu J., } \\
\text { Sarkis J., Xue B. }\end{array}$ & $\begin{array}{c}\text { Towards a national circular } \\
\text { economy indicator system in } \\
\text { China: An evaluation and } \\
\text { critical analysis }\end{array}$ & $\begin{array}{l}\text { Journal of Cleaner } \\
\text { Production }\end{array}$ & 2012 & 23 & 1 & 216 & 224 & 10.1016/j.jclepro.2011.07.005 \\
\hline $\begin{array}{l}\text { Laybourn P., } \\
\text { Lombardi D.R. }\end{array}$ & $\begin{array}{l}\text { Industrial Symbiosis in } \\
\text { European Policy: Overview of } \\
\text { Recent Progress }\end{array}$ & $\begin{array}{l}\text { Journal of Industrial } \\
\text { Ecology }\end{array}$ & 2012 & 16 & 1 & 11 & 12 & 10.1111/j.1530-9290.2011.00451.x \\
\hline $\begin{array}{l}\text { Lombardi D.R., } \\
\text { Lyons D., Shi H., } \\
\text { Agarwal A. }\end{array}$ & $\begin{array}{c}\text { Industrial Symbiosis: Testing } \\
\text { the Boundaries and Advancing } \\
\text { Knowledge }\end{array}$ & $\begin{array}{c}\text { Journal of Industrial } \\
\text { Ecology }\end{array}$ & 2012 & 16 & 1 & 2 & 7 & 10.1111/j.1530-9290.2012.00455.x \\
\hline $\begin{array}{l}\text { Paquin R.L., } \\
\text { Howard- } \\
\text { Grenville J. }\end{array}$ & $\begin{array}{l}\text { The Evolution of Facilitated } \\
\text { Industrial Symbiosis }\end{array}$ & $\begin{array}{c}\text { Journal of Industrial } \\
\text { Ecology }\end{array}$ & 2012 & 16 & 1 & 83 & 93 & 10.1111/j.1530-9290.2011.00437.x \\
\hline $\begin{array}{l}\text { Jensen P.D., } \\
\text { Basson L., } \\
\text { Hellawell E.E., } \\
\text { Leach M. }\end{array}$ & $\begin{array}{l}\text { 'Habitat' Suitability Index } \\
\text { Mapping for Industrial } \\
\text { Symbiosis Planning }\end{array}$ & $\begin{array}{l}\text { Journal of Industrial } \\
\text { Ecology }\end{array}$ & 2012 & 16 & 1 & 38 & 50 & 10.1111/j.1530-9290.2011.00438.x \\
\hline $\begin{array}{l}\text { Salmi O., } \\
\text { Hukkinen J., } \\
\text { Heino J., Pajunen } \\
\text { N., Wierink M. }\end{array}$ & $\begin{array}{l}\text { Governing the Interplay } \\
\text { between Industrial Ecosystems } \\
\text { and Environmental Regulation: } \\
\text { Heavy Industries in the Gulf of } \\
\text { Bothnia in Finland and Sweden }\end{array}$ & $\begin{array}{l}\text { Journal of Industrial } \\
\text { Ecology }\end{array}$ & 2012 & 16 & 1 & 119 & 128 & 10.1111/j.1530-9290.2011.00403.x \\
\hline $\begin{array}{l}\text { Chertow M., } \\
\text { Ehrenfeld J. }\end{array}$ & $\begin{array}{c}\text { Organizing Self-Organizing } \\
\text { Systems: Toward a Theory of } \\
\text { Industrial Symbiosis }\end{array}$ & $\begin{array}{c}\text { Journal of Industrial } \\
\text { Ecology }\end{array}$ & 2012 & 16 & 1 & 13 & 27 & 10.1111/j.1530-9290.2011.00450.x \\
\hline $\begin{array}{l}\text { Lombardi D.R., } \\
\text { Laybourn P. }\end{array}$ & $\begin{array}{c}\text { Redefining Industrial } \\
\text { Symbiosis: Crossing } \\
\text { Academic-Practitioner } \\
\text { Boundaries } \\
\end{array}$ & $\begin{array}{l}\text { Journal of Industrial } \\
\text { Ecology }\end{array}$ & 2012 & 16 & 1 & 28 & 37 & 10.1111/j.1530-9290.2011.00444.x \\
\hline
\end{tabular}

APPLIED ECOLOGY AND ENVIRONMENTAL RESEARCH 17(1):1159-1221.

http://www.aloki.hu • ISSN 15891623 (Print) • ISSN 17850037 (Online)

DOI: http://dx.doi.org/10.15666/aeer/1701_11591221

(c) 2019, ALÖKI Kft., Budapest, Hungary 


\begin{tabular}{|c|c|c|c|c|c|c|c|c|}
\hline Author Name(s) & Title & Source Title & Year & Vol. & Issue & P. Start & P. End & Doi \\
\hline $\begin{array}{c}\text { Chen X., Fujita } \\
\text { T., Ohnishi S., } \\
\text { Fujii M., Geng Y. }\end{array}$ & $\begin{array}{c}\text { The Impact of Scale, Recycling } \\
\text { Boundary, and Type of Waste } \\
\text { on Symbiosis and Recycling: } \\
\text { An Empirical Study of } \\
\text { Japanese Eco-Towns }\end{array}$ & $\begin{array}{c}\text { Journal of Industrial } \\
\text { Ecology }\end{array}$ & 2012 & 16 & 1 & 129 & 141 & 10.1111/j.1530-9290.2011.00422.x \\
\hline $\begin{array}{l}\text { Mattila T., } \\
\text { Lehtoranta S., } \\
\text { Sokka L., } \\
\text { Melanen M., } \\
\text { Nissinen A. } \\
\end{array}$ & $\begin{array}{c}\text { Methodological Aspects of } \\
\text { Applying Life Cycle } \\
\text { Assessment to Industrial } \\
\text { Symbioses }\end{array}$ & $\begin{array}{c}\text { Journal of Industrial } \\
\text { Ecology }\end{array}$ & 2012 & 16 & 1 & 51 & 60 & 10.1111/j.1530-9290.2011.00443.x \\
\hline $\begin{array}{l}\text { Ashton W.S., } \\
\text { Bain A.C. }\end{array}$ & $\begin{array}{l}\text { Assessing the "Short Mental } \\
\text { Distance" in Eco-Industrial } \\
\text { Networks }\end{array}$ & $\begin{array}{l}\text { Journal of Industrial } \\
\text { Ecology }\end{array}$ & 2012 & 16 & 1 & 70 & 82 & 10.1111/j.1530-9290.2011.00453.x \\
\hline $\begin{array}{l}\text { Boons F., } \\
\text { Spekkink W. }\end{array}$ & $\begin{array}{l}\text { Levels of Institutional Capacity } \\
\text { and Actor Expectations about } \\
\text { Industrial Symbiosis: Evidence } \\
\text { from the Dutch Stimulation } \\
\text { Program 1999-2004 }\end{array}$ & $\begin{array}{c}\text { Journal of Industrial } \\
\text { Ecology }\end{array}$ & 2012 & 16 & 1 & 61 & 69 & 10.1111/j.1530-9290.2011.00432.x \\
\hline $\begin{array}{l}\text { Zhou L., Hu S.- } \\
\text { Y., Li Y., Jin Y., } \\
\text { Zhang X. }\end{array}$ & $\begin{array}{l}\text { Modeling and Optimization of } \\
\text { a Coal-Chemical Eco-industrial } \\
\text { System in China }\end{array}$ & $\begin{array}{l}\text { Journal of Industrial } \\
\text { Ecology }\end{array}$ & 2012 & 16 & 1 & 105 & 118 & 10.1111/j.1530-9290.2012.00447.x \\
\hline $\begin{array}{c}\text { Ferrer G., } \\
\text { Cortezia S., } \\
\text { Neumann J.M. }\end{array}$ & $\begin{array}{c}\text { Green City: Environmental and } \\
\text { Social Responsibility in an } \\
\text { Industrial Cluster } \\
\end{array}$ & $\begin{array}{c}\text { Journal of Industrial } \\
\text { Ecology }\end{array}$ & 2012 & 16 & 1 & 142 & 152 & 10.1111/j.1530-9290.2011.00442.x \\
\hline $\begin{array}{l}\text { Brent G.F., Allen } \\
\text { D.J., Eichler } \\
\text { B.R., Petrie J.G., } \\
\text { Mann J.P., } \\
\text { Haynes B.S. } \\
\end{array}$ & $\begin{array}{l}\text { Mineral Carbonation as the } \\
\text { Core of an Industrial Symbiosis } \\
\text { for Energy-Intensive Minerals } \\
\text { Conversion }\end{array}$ & $\begin{array}{c}\text { Journal of Industrial } \\
\text { Ecology }\end{array}$ & 2012 & 16 & 1 & 94 & 104 & 10.1111/j.1530-9290.2011.00368.x \\
\hline $\begin{array}{l}\text { Liu C., Ma C., } \\
\text { Zhang K. }\end{array}$ & $\begin{array}{l}\text { Going beyond the sectoral } \\
\text { boundary: A key stage in the } \\
\text { development of a regional } \\
\text { industrial ecosystem }\end{array}$ & $\begin{array}{c}\text { Journal of Cleaner } \\
\text { Production }\end{array}$ & 2012 & 22 & 1 & 42 & 49 & 10.1016/j.jclepro.2011.09.022 \\
\hline
\end{tabular}




\begin{tabular}{|c|c|c|c|c|c|c|c|c|}
\hline Author Name(s) & Title & Source Title & Year & Vol. & Issue & P. Start & P. End & Doi \\
\hline $\begin{array}{l}\text { Usón S., Valero } \\
\text { A., Valero A., } \\
\text { Costa J. }\end{array}$ & $\begin{array}{l}\text { Thermoeconomic fuel impact } \\
\text { approach for assessing } \\
\text { resources savings in industrial } \\
\text { symbiosis: Application to } \\
\text { kalundborg ecoindustrial park }\end{array}$ & $\begin{array}{c}\text { Proceedings of the 25th } \\
\text { International } \\
\text { Conference on } \\
\text { Efficiency, Cost, } \\
\text { Optimization and } \\
\text { Simulation of Energy } \\
\text { Conversion Systems } \\
\text { and Processes, ECOS } \\
2012 \\
\end{array}$ & 2012 & 3 & & 346 & 356 & \\
\hline $\begin{array}{l}\text { Valero A., Usón } \\
\text { S., Costa J. }\end{array}$ & $\begin{array}{c}\text { Exergy analysis of the } \\
\text { industrial symbiosis model in } \\
\text { Kalundborg }\end{array}$ & $\begin{array}{c}\text { Proceedings of the 25th } \\
\text { International } \\
\text { Conference on } \\
\text { Efficiency, Cost, } \\
\text { Optimization and } \\
\text { Simulation of Energy } \\
\text { Conversion Systems } \\
\text { and Processes, ECOS } \\
2012\end{array}$ & 2012 & 1 & & 406 & 416 & \\
\hline $\begin{array}{c}\text { Gregson N., } \\
\text { Crang M., } \\
\text { Ahamed F.U., } \\
\text { Akter N., Ferdous } \\
\text { R., Foisal S., } \\
\text { Hudson R. }\end{array}$ & $\begin{array}{c}\text { Territorial agglomeration and } \\
\text { industrial symbiosis: } \\
\text { Sitakunda-Bhatiary, } \\
\text { Bangladesh, as a secondary } \\
\text { processing complex }\end{array}$ & Economic Geography & 2012 & 88 & 1 & 37 & 58 & 10.1111/j.1944-8287.2011.01138.x \\
\hline $\begin{array}{l}\text { Li J., Wang Y., } \\
\text { Zhou M. }\end{array}$ & $\begin{array}{l}\text { Application of emergy theory } \\
\text { in industrial ecosystem analysis }\end{array}$ & $\begin{array}{l}\text { Advanced Materials } \\
\text { Research }\end{array}$ & 2012 & $\begin{array}{l}361- \\
363\end{array}$ & & 1249 & 1254 & $\begin{array}{c}\text { 10.4028/www.scientific.net/AMR.361- } \\
363.1249\end{array}$ \\
\hline Yang L., Tong L. & $\begin{array}{l}\text { Research of typical EIPs based } \\
\text { on the social network analysis }\end{array}$ & $\begin{array}{l}\text { Shengtai Xuebao/ Acta } \\
\text { Ecologica Sinica }\end{array}$ & 2012 & 32 & 13 & 4236 & 4245 & \\
\hline $\begin{array}{l}\text { Martin M., } \\
\text { Svensson N., } \\
\text { Fonseca J., } \\
\text { Eklund M. }\end{array}$ & $\begin{array}{l}\text { Quantifying the environmental } \\
\text { performance of integrated } \\
\text { bioethanol and biogas } \\
\text { production }\end{array}$ & Renewable Energy & 2013 & 61 & & 109 & 116 & 10.1016/j.renene.2012.09.058 \\
\hline Li G. & $\begin{array}{l}\text { A paradigm of constructing } \\
\text { industrial symbiosis and } \\
\text { coupling in China's county- } \\
\text { region economic sustainable } \\
\text { development }\end{array}$ & $\begin{array}{l}\text { Sustainable Practices: } \\
\text { Concepts, } \\
\text { Methodologies, Tools, } \\
\text { and Applications }\end{array}$ & 2013 & 3 & & 1218 & 1231 & 10.4018/978-1-4666-4852-4.ch068 \\
\hline
\end{tabular}




\begin{tabular}{|c|c|c|c|c|c|c|c|c|}
\hline Author Name(s) & Title & Source Title & Year & Vol. & Issue & P. Start & P. End & Doi \\
\hline MacLachlan I. & $\begin{array}{c}\text { Kwinana Industrial Area: } \\
\text { Agglomeration economies and } \\
\text { industrial symbiosis on } \\
\text { Western Australia's Cockburn } \\
\text { Sound }\end{array}$ & Australian Geographer & 2013 & 44 & 4 & 383 & 400 & $10.1080 / 00049182.2013 .852505$ \\
\hline $\begin{array}{c}\text { Raafat T., } \\
\text { Trokanas N., } \\
\text { Cecelja F., Bimi } \\
\text { X. }\end{array}$ & $\begin{array}{l}\text { An ontological approach } \\
\text { towards enabling processing } \\
\text { technologies participation in } \\
\text { industrial symbiosis }\end{array}$ & $\begin{array}{c}\text { Computers and } \\
\text { Chemical Engineering }\end{array}$ & 2013 & 59 & & 33 & 46 & 10.1016/j.compchemeng.2013.03.022 \\
\hline Li J., Gao Y. & $\begin{array}{l}\text { Research on eco-industry } \\
\text { symbiosis system based on } \\
\text { complex network }\end{array}$ & $\begin{array}{c}\text { Proceedings of } 2012 \\
\text { 3rd International Asia } \\
\text { Conference on } \\
\text { Industrial Engineering } \\
\text { and Management } \\
\text { Innovation, IEMI 2012 }\end{array}$ & 2013 & & & 759 & 769 & $10.1007 / 978-3-642-33012-4-76$ \\
\hline $\begin{array}{l}\text { Marinos-Kouris } \\
\text { D., Mourtsiadis } \\
\text { A. }\end{array}$ & $\begin{array}{c}\text { Environmental limits of } \\
\text { industrial symbiosis: The case } \\
\text { of aluminium eco-industrial } \\
\text { network }\end{array}$ & $\begin{array}{c}\text { Fresenius } \\
\text { Environmental Bulletin }\end{array}$ & 2013 & 22 & 12 & 3549 & 3557 & \\
\hline $\begin{array}{l}\text { Valero A., Usón } \\
\text { S., Torres C., } \\
\text { Valero A., } \\
\text { Agudelo A., } \\
\text { Costa J. } \\
\end{array}$ & $\begin{array}{l}\text { Thermoeconomic tools for the } \\
\text { analysis of eco-industrial parks }\end{array}$ & Energy & 2013 & 62 & & 62 & 72 & 10.1016/j.energy.2013.07.014 \\
\hline $\begin{array}{l}\text { [No author name } \\
\text { available] }\end{array}$ & $\begin{array}{c}\text { IFIP WG 5.7 International } \\
\text { Conference on Advances in } \\
\text { Production Management } \\
\text { Systems, APMS } 2012\end{array}$ & $\begin{array}{l}\text { IFIP Advances in } \\
\text { Information and } \\
\text { Communication } \\
\text { Technology }\end{array}$ & 2013 & 397 & $\begin{array}{c}\text { PART } \\
1\end{array}$ & & & \\
\hline $\begin{array}{c}\text { Dong L., Zhang } \\
\text { H., Fujita T., } \\
\text { Ohnishi S., Li H., } \\
\text { Fujii M., Dong H. }\end{array}$ & $\begin{array}{l}\text { Environmental and economic } \\
\text { gains of industrial symbiosis } \\
\text { for Chinese iron/steel industry: } \\
\text { Kawasaki's experience and } \\
\text { practice in Liuzhou and Jinan }\end{array}$ & $\begin{array}{l}\text { Journal of Cleaner } \\
\text { Production }\end{array}$ & 2013 & 59 & & 226 & 238 & 10.1016/j.jclepro.2013.06.048 \\
\hline $\begin{array}{l}\text { Paquin R.L., } \\
\text { Howard- } \\
\text { Grenville J. }\end{array}$ & $\begin{array}{l}\text { Blind Dates and Arranged } \\
\text { Marriages: Longitudinal } \\
\text { Processes of Network } \\
\text { Orchestration }\end{array}$ & Organization Studies & 2013 & 34 & 11 & 1623 & 1653 & $10.1177 / 0170840612470230$ \\
\hline
\end{tabular}

APPLIED ECOLOGY AND ENVIRONMENTAL RESEARCH 17(1):1159-1221.

http://www.aloki.hu • ISSN 15891623 (Print) • ISSN 17850037 (Online)

DOI: http://dx.doi.org/10.15666/aeer/1701_11591221

๑ 2019, ALÖKI Kft., Budapest, Hungary 


\begin{tabular}{|c|c|c|c|c|c|c|c|c|}
\hline Author Name(s) & Title & Source Title & Year & Vol. & Issue & P. Start & P. End & Doi \\
\hline $\begin{array}{l}\text { Liu G.-F., Chen } \\
\text { F.-D. }\end{array}$ & $\begin{array}{l}\text { NISP-based research on the } \\
\text { system structure of urban } \\
\text { symbiosis network in China }\end{array}$ & $\begin{array}{l}\text { Applied Mechanics and } \\
\text { Materials }\end{array}$ & 2013 & $\begin{array}{l}427- \\
429\end{array}$ & & 2923 & 2927 & $\begin{array}{l}\text { 10.4028/www.scientific.net/AMM.427- } \\
429.2923\end{array}$ \\
\hline $\begin{array}{l}\text { Gao X.L., Li } \\
\text { R.Q., Li R. }\end{array}$ & $\begin{array}{l}\text { Study on byproducts recycling } \\
\text { in eco-industrial parks }\end{array}$ & $\begin{array}{c}\text { Advanced Materials } \\
\text { Research } \\
\end{array}$ & 2013 & 788 & & 288 & 292 & 10.4028/www.scientific.net/AMR.788.288 \\
\hline $\begin{array}{l}\text { Liao M.-I., Ma } \\
\text { H.-W. }\end{array}$ & $\begin{array}{l}\text { The potential environmental } \\
\text { gains from industrial } \\
\text { symbiosis: Evaluation of } \mathrm{CO} 2 \\
\text { reduction through a crucial by- } \\
\text { product }\end{array}$ & $\begin{array}{l}\text { International Journal of } \\
\text { Applied Environmental } \\
\text { Sciences }\end{array}$ & 2013 & 8 & 2 & 129 & 136 & \\
\hline $\begin{array}{l}\text { Shi X.Q., Li } \\
\text { X.N., Yang J.X. }\end{array}$ & $\begin{array}{c}\text { Eco-management benefit } \\
\text { analysis of industrial resources } \\
\text { from life cycle perspective: A } \\
\text { case study of a virtual } \\
\text { symbiosis network }\end{array}$ & $\begin{array}{l}\text { Shengtai Xuebao/ Acta } \\
\text { Ecologica Sinica }\end{array}$ & 2013 & 33 & 19 & 6398 & 6410 & $10.5846 / \mathrm{stxb} 201304180738$ \\
\hline $\begin{array}{l}\text { Dong L., Fujita } \\
\text { T., Zhang H., Dai } \\
\text { M., Fujii M., } \\
\text { Ohnishi S., Geng } \\
\text { Y., Liu Z. } \\
\end{array}$ & $\begin{array}{l}\text { Promoting low-carbon city } \\
\text { through industrial symbiosis: A } \\
\text { case in China by applying } \\
\text { HPIMO model }\end{array}$ & Energy Policy & 2013 & 61 & & 864 & 873 & 10.1016/j.enpol.2013.06.084 \\
\hline $\begin{array}{l}\text { Marinos-Kouris } \\
\text { D., Mourtsiadis } \\
\text { A. }\end{array}$ & $\begin{array}{c}\text { Industrial symbiosis in Greece: } \\
\text { A study of spatial allocation } \\
\text { patterns }\end{array}$ & $\begin{array}{c}\text { Fresenius } \\
\text { Environmental Bulletin }\end{array}$ & 2013 & 22 & $7 \mathrm{~B}$ & 2174 & 2181 & \\
\hline $\begin{array}{l}\text { Zhang H., Dong } \\
\text { L., Li H., Fujita } \\
\text { T., Ohnishi S., } \\
\text { Tang Q. }\end{array}$ & $\begin{array}{c}\text { Analysis of low-carbon } \\
\text { industrial symbiosis technology } \\
\text { for carbon mitigation in a } \\
\text { Chinese iron/steel industrial } \\
\text { park: A case study with carbon } \\
\text { flow analysis } \\
\end{array}$ & Energy Policy & 2013 & 61 & & 1400 & 1411 & 10.1016/j.enpol.2013.05.066 \\
\hline $\begin{array}{l}\text { Gu C., Leveneur } \\
\text { S., Estel L., } \\
\text { Yassine A. }\end{array}$ & $\begin{array}{c}\text { Industrial symbiosis } \\
\text { optimization control model for } \\
\text { the exchanges of the } \\
\text { material/energy flows in an } \\
\text { industrial production park }\end{array}$ & $\begin{array}{l}\text { IFAC Proceedings } \\
\text { Volumes (IFAC- } \\
\text { PapersOnline) }\end{array}$ & 2013 & & & 1015 & 1020 & 10.3182/20130619-3-RU-3018.00182 \\
\hline $\begin{array}{l}\text { Liu G.-S., Xu S.- } \\
\text { Q., Sun Y.-W., } \\
\text { Han J.-Y. }\end{array}$ & $\begin{array}{l}\text { Eco-industrial symbiosis } \\
\text { network equilibrium model }\end{array}$ & $\begin{array}{c}\text { Beijing Keji Daxue } \\
\text { Xuebao/Journal of } \\
\text { University of Science } \\
\text { and Technology Beijing }\end{array}$ & 2013 & 35 & 9 & 1221 & 1229 & \\
\hline
\end{tabular}




\begin{tabular}{|c|c|c|c|c|c|c|c|c|}
\hline Author Name(s) & Title & Source Title & Year & Vol. & Issue & P. Start & P. End & Doi \\
\hline $\begin{array}{l}\text { Eckelman M.J., } \\
\text { Chertow M.R. }\end{array}$ & $\begin{array}{l}\text { Life cycle energy and } \\
\text { environmental benefits of a US } \\
\text { industrial symbiosis }\end{array}$ & $\begin{array}{l}\text { International Journal of } \\
\text { Life Cycle Assessment }\end{array}$ & 2013 & 18 & 8 & 1524 & 1532 & $10.1007 / \mathrm{s} 11367-013-0601-5$ \\
\hline $\begin{array}{l}\text { Ng R.T.L., Ng } \\
\text { D.K.S., Tan R.R. }\end{array}$ & $\begin{array}{c}\text { Systematic approach for } \\
\text { synthesis of integrated palm oil } \\
\text { processing complex. Part 2: } \\
\text { Multiple owners }\end{array}$ & $\begin{array}{c}\text { Industrial and } \\
\text { Engineering Chemistry } \\
\text { Research }\end{array}$ & 2013 & 52 & 30 & 10221 & 10235 & $10.1021 / \mathrm{ie} 400846 \mathrm{~g}$ \\
\hline $\begin{array}{l}\text { Ng R.T.L., } \mathrm{Ng} \\
\text { D.K.S. }\end{array}$ & $\begin{array}{l}\text { Systematic approach for } \\
\text { synthesis of integrated palm oil } \\
\text { processing complex. Part 1: } \\
\text { Single owner }\end{array}$ & $\begin{array}{c}\text { Industrial and } \\
\text { Engineering Chemistry } \\
\text { Research }\end{array}$ & 2013 & 52 & 30 & 10206 & 10220 & 10.1021/ie302926q \\
\hline $\begin{array}{c}\text { Xiong W., Wang } \\
\text { J., Tang W., } \\
\text { Kong W., Zeng } \\
\text { Z., Ouyang J., } \\
\text { Liu M., Wang G., } \\
\text { Huang M., Xiong } \\
\text { D. } \\
\end{array}$ & $\begin{array}{c}\text { Establishment of integrative } \\
\text { circular agro-ecology system } \\
\text { for multiple agricultural } \\
\text { industries in Three Gorges } \\
\text { Reservoir Area }\end{array}$ & $\begin{array}{c}\text { Nongye Gongcheng } \\
\text { Xuebao/Transactions of } \\
\text { the Chinese Society of } \\
\text { Agricultural } \\
\text { Engineering }\end{array}$ & 2013 & 29 & 14 & 203 & 209 & 10.3969/j.issn.1002-6819.2013.14.026 \\
\hline $\begin{array}{c}\text { Trokanas N., } \\
\text { Raafat T., Cecelja } \\
\text { F., Kokossis A. } \\
\end{array}$ & $\begin{array}{l}\text { OFIS - Ontological Framework } \\
\text { for Industrial Symbiosis }\end{array}$ & $\begin{array}{c}\text { Computer Aided } \\
\text { Chemical Engineering }\end{array}$ & 2013 & 32 & & 523 & 528 & 10.1016/B978-0-444-63234-0.50088-9 \\
\hline Zhu J., Ruth M. & $\begin{array}{l}\text { Exploring the resilience of } \\
\text { industrial ecosystems }\end{array}$ & $\begin{array}{c}\text { Journal of } \\
\text { Environmental } \\
\text { Management } \\
\end{array}$ & 2013 & 122 & & 65 & 75 & 10.1016/j.jenvman.2013.02.052 \\
\hline $\begin{array}{l}\text { Zhang Y., Zheng } \\
\text { H., Chen B., } \\
\text { Yang N. }\end{array}$ & $\begin{array}{l}\text { Social network analysis and } \\
\text { network connectedness } \\
\text { analysis for industrial } \\
\text { symbiotic systems: Model } \\
\text { development and case study }\end{array}$ & $\begin{array}{l}\text { Frontiers of Earth } \\
\text { Science }\end{array}$ & 2013 & 7 & 2 & 169 & 181 & $10.1007 / \mathrm{s} 11707-012-0349-4$ \\
\hline $\begin{array}{l}\text { Zhang H., Dong } \\
\text { L., Li H.-Q., } \\
\text { Chen B., Tang } \\
\text { Q., Fujita T. }\end{array}$ & $\begin{array}{l}\text { Investigation of the residual } \\
\text { heat recovery and carbon } \\
\text { emission mitigation potential in } \\
\text { a Chinese steelmaking plant: A } \\
\text { hybrid material/energy flow } \\
\text { analysis case study }\end{array}$ & $\begin{array}{l}\text { Sustainable Energy } \\
\text { Technologies and } \\
\text { Assessments }\end{array}$ & 2013 & 2 & 1 & 67 & 80 & 10.1016/j.seta.2013.03.003 \\
\hline
\end{tabular}




\begin{tabular}{|c|c|c|c|c|c|c|c|c|}
\hline Author Name(s) & Title & Source Title & Year & Vol. & Issue & P. Start & P. End & Doi \\
\hline $\begin{array}{l}\text { Husgafvel R., } \\
\text { Watkins G., } \\
\text { Linkosalmi L., } \\
\text { Dahl O. }\end{array}$ & $\begin{array}{c}\text { Review of sustainability } \\
\text { management initiatives within } \\
\text { Finnish forest products industry } \\
\text { companies - Translating Eu } \\
\text { level steering into proactive } \\
\text { initiatives } \\
\end{array}$ & $\begin{array}{l}\text { Resources, } \\
\text { Conservation and } \\
\text { Recycling }\end{array}$ & 2013 & 76 & & 1 & 11 & 10.1016/j.resconrec.2013.04.006 \\
\hline $\begin{array}{l}\text { Pajunen N., } \\
\text { Watkins G., } \\
\text { Husgafvel R., } \\
\text { Heiskanen K., } \\
\text { Dahl O. }\end{array}$ & $\begin{array}{l}\text { The challenge to overcome } \\
\text { institutional barriers in the } \\
\text { development of industrial } \\
\text { residue based novel symbiosis } \\
\text { products - Experiences from } \\
\text { Finnish process industry }\end{array}$ & Minerals Engineering & 2013 & $46-47$ & & 144 & 156 & 10.1016/j.mineng.2013.03.008 \\
\hline $\begin{array}{l}\text { Hara K., Uwasu } \\
\text { M., Yabar H., } \\
\text { Zhang H. }\end{array}$ & $\begin{array}{l}\text { Urban development and its } \\
\text { impacts on energy and resource } \\
\text { consumptions in the Yangtze } \\
\text { river delta: Trends and future } \\
\text { prospects }\end{array}$ & $\begin{array}{c}\text { Yangtze River: } \\
\text { Geography, Pollution } \\
\text { and Environmental } \\
\text { Implications }\end{array}$ & 2013 & & & 121 & 127 & \\
\hline Boons F. & $\begin{array}{l}\text { Ecological Modernization and } \\
\text { Industrial Ecology }\end{array}$ & $\begin{array}{l}\text { The Handbook of } \\
\text { Global Companies }\end{array}$ & 2013 & & & 388 & 402 & 10.1002/9781118326152.ch23 \\
\hline Feng L., Di J.H. & $\begin{array}{l}\text { A new model of industrial } \\
\text { symbiosis optimization }\end{array}$ & $\begin{array}{l}\text { Hydraulic Engineering - } \\
\text { Proceedings of the } 2012 \\
\text { SREE Conference on } \\
\text { Hydraulic Engineering, } \\
\text { CHE } 2012 \text { and 2nd } \\
\text { SREE Workshop on } \\
\text { Environment and Safety } \\
\text { Engineering, WESE } \\
2012\end{array}$ & 2013 & & & 365 & 370 & \\
\hline Spekkink W. & $\begin{array}{l}\text { Institutional capacity building } \\
\text { for industrial symbiosis in the } \\
\text { Canal Zone of Zeeland in the } \\
\text { Netherlands: A process } \\
\text { analysis } \\
\end{array}$ & $\begin{array}{l}\text { Journal of Cleaner } \\
\text { Production }\end{array}$ & 2013 & 52 & & 342 & 355 & 10.1016/j.jclepro.2013.02.025 \\
\hline
\end{tabular}




\begin{tabular}{|c|c|c|c|c|c|c|c|c|}
\hline Author Name(s) & Title & Source Title & Year & Vol. & Issue & P. Start & P. End & Doi \\
\hline Boons F. & $\begin{array}{l}\text { Industrial Symbiosis and the } \\
\text { Chemical Industry: Between } \\
\text { Exploration and Exploitation }\end{array}$ & $\begin{array}{c}\text { Management Principles } \\
\text { of Sustainable } \\
\text { Industrial Chemistry: } \\
\text { Theories, Concepts and } \\
\text { Industrial Examples for } \\
\text { Achieving Sustainable } \\
\text { Chemical Products and } \\
\text { Processes from a Non- } \\
\text { Technological } \\
\text { Viewpoint }\end{array}$ & 2013 & & & 131 & 145 & 10.1002/9783527649488.ch9 \\
\hline $\begin{array}{l}\text { Montastruc L., } \\
\text { Boix M., } \\
\text { Pibouleau L., } \\
\text { Azzaro-Pantel C., } \\
\text { Domenech S. } \\
\end{array}$ & $\begin{array}{l}\text { On the flexibility of an eco- } \\
\text { industrial park (EIP) for } \\
\text { managing industrial water }\end{array}$ & $\begin{array}{l}\text { Journal of Cleaner } \\
\text { Production }\end{array}$ & 2013 & 43 & & 1 & 11 & 10.1016/j.jclepro.2012.12.039 \\
\hline $\begin{array}{l}\text { Watkins G., } \\
\text { Husgafvel R., } \\
\text { Pajunen N., Dahl } \\
\text { O., Heiskanen K. }\end{array}$ & $\begin{array}{l}\text { Overcoming institutional } \\
\text { barriers in the development of } \\
\text { novel process industry residue } \\
\text { based symbiosis products - } \\
\text { Case study at the EU level }\end{array}$ & Minerals Engineering & 2013 & 41 & & 31 & 40 & 10.1016/j.mineng.2012.10.003 \\
\hline $\begin{array}{l}\text { Termsinvanich } \\
\text { P., Thadaniti S., } \\
\text { Wiwattanadate D. }\end{array}$ & $\begin{array}{l}\text { Conceptual model for effective } \\
\text { implementation of industrial } \\
\text { symbiosis: A case study of } \\
\text { Mab-Ta-Phut industrial estate }\end{array}$ & $\begin{array}{l}\text { Mediterranean Journal } \\
\text { of Social Sciences }\end{array}$ & 2013 & 4 & 1 & 133 & 139 & 10.5901/mjss.2013.v4n1p133 \\
\hline $\begin{array}{l}\text { Albino V., } \\
\text { Garavelli A.C., } \\
\text { Romano V.A. }\end{array}$ & $\begin{array}{l}\text { A Classification of industrial } \\
\text { symbiosis networks: A focus } \\
\text { on materials and energy } \\
\text { recovery }\end{array}$ & $\begin{array}{l}\text { IFIP Advances in } \\
\text { Information and } \\
\text { Communication } \\
\text { Technology } \\
\end{array}$ & 2013 & 397 & $\begin{array}{c}\text { PART } \\
1\end{array}$ & 216 & 223 & 10.1007/978-3-642-40352-1_28 \\
\hline $\begin{array}{l}\text { Wang G., Feng } \\
\text { X., Chu K.H. }\end{array}$ & $\begin{array}{l}\text { A novel approach for stability } \\
\text { analysis of industrial symbiosis } \\
\text { systems }\end{array}$ & $\begin{array}{l}\text { Journal of Cleaner } \\
\text { Production }\end{array}$ & 2013 & 39 & & 9 & 16 & 10.1016/j.jclepro.2012.08.031 \\
\hline $\begin{array}{l}\text { Antonopoulos } \\
\text { I.S., Zouboulis } \\
\text { A.I., } \\
\text { Karagiannidis A., } \\
\text { Samaras P. }\end{array}$ & $\begin{array}{l}\text { Applying dpsir analysis as a } \\
\text { decision support tool for } \\
\text { fostering industrial symbiosis } \\
\text { concept }\end{array}$ & $\begin{array}{c}\text { Fresenius } \\
\text { Environmental Bulletin }\end{array}$ & 2013 & 22 & $12 \mathrm{C}$ & 3830 & 3839 & \\
\hline
\end{tabular}

APPLIED ECOLOGY AND ENVIRONMENTAL RESEARCH 17(1):1159-1221.

http://www.aloki.hu • ISSN 15891623 (Print) • ISSN 17850037 (Online)

DOI: http://dx.doi.org/10.15666/aeer/1701_11591221

(c) 2019, ALÖKI Kft., Budapest, Hungary 


\begin{tabular}{|c|c|c|c|c|c|c|c|c|}
\hline Author Name(s) & $\begin{array}{c}\text { Title } \\
\end{array}$ & Source Title & Year & Vol. & Issue & P. Start & P. End & Doi \\
\hline $\begin{array}{l}\text { Ng W.P.Q., Lam } \\
\text { H.L. }\end{array}$ & $\begin{array}{c}\text { Sustainable supply network } \\
\text { design through optimisation } \\
\text { with clustering technique } \\
\text { integration }\end{array}$ & $\begin{array}{c}\text { Chemical Engineering } \\
\text { Transactions }\end{array}$ & 2013 & 35 & & 661 & 666 & 10.3303/CET1335110 \\
\hline Rankin W.J. & $\begin{array}{c}\text { Towards zero waste production } \\
\text { in the minerals and metals } \\
\text { sector }\end{array}$ & TMS Annual Meeting & 2013 & & & 392 & 403 & \\
\hline $\begin{array}{l}\text { Gu C., Leveneur } \\
\text { S., Estel L., } \\
\text { Yassine A. }\end{array}$ & $\begin{array}{c}\text { Modeling and optimization of } \\
\text { material/energy flow } \\
\text { exchanges in an eco-industrial } \\
\text { park }\end{array}$ & Energy Procedia & 2013 & 36 & & 243 & 252 & 10.1016/j.egypro.2013.07.028 \\
\hline $\begin{array}{l}\text { Mohammed F.A., } \\
\text { Yao H.M., } \\
\text { OludayoTadé M., } \\
\text { Biswas W. }\end{array}$ & $\begin{array}{c}\text { A framework for synergy } \\
\text { evaluation and development in } \\
\text { heavy industries }\end{array}$ & $\begin{array}{l}\text { Re-Engineering } \\
\text { Manufacturing for } \\
\text { Sustainability - } \\
\text { Proceedings of the 20th } \\
\text { CIRP International } \\
\text { Conference on Life } \\
\text { Cycle Engineering }\end{array}$ & 2013 & & & 591 & 595 & \\
\hline $\begin{array}{l}\text { Gu C., Estel L., } \\
\text { Yassine A., } \\
\text { Leveneur S. }\end{array}$ & $\begin{array}{l}\text { A multiobjective optimization } \\
\text { model for designing and } \\
\text { optimizing an ecological } \\
\text { industrial park } \\
\end{array}$ & $\begin{array}{c}\text { Proceedings - } \\
\text { International } \\
\text { Conference on Natural } \\
\text { Computation } \\
\end{array}$ & 2013 & & & 595 & 600 & 10.1109/ICNC.2013.6818046 \\
\hline $\begin{array}{l}\text { Wood B.M., } \\
\text { Jader L.R., } \\
\text { Schendel F.J., } \\
\text { Hahn N.J., } \\
\text { Valentas K.J., } \\
\text { Mcnamara P.J., } \\
\text { Novak P.M., } \\
\text { Heilmann S.M. } \\
\end{array}$ & $\begin{array}{l}\text { Industrial symbiosis: Corn } \\
\text { ethanol fermentation, } \\
\text { hydrothermal carbonization, } \\
\text { and anaerobic digestion }\end{array}$ & $\begin{array}{l}\text { Biotechnology and } \\
\text { Bioengineering }\end{array}$ & 2013 & 110 & 10 & 2624 & 2632 & 10.1002/bit.24924 \\
\hline $\begin{array}{c}\text { Kreiger M.A., } \\
\text { Shonnard D.R., } \\
\text { Pearce J.M. }\end{array}$ & $\begin{array}{l}\text { Life cycle analysis of silane } \\
\text { recycling in amorphous silicon- } \\
\text { based solar photovoltaic } \\
\text { manufacturing }\end{array}$ & $\begin{array}{l}\text { Resources, } \\
\text { Conservation and } \\
\text { Recycling }\end{array}$ & 2013 & 70 & & 44 & 49 & 10.1016/j.resconrec.2012.10.002 \\
\hline $\begin{array}{l}\text { Jung S., Dodbiba } \\
\text { G., Chae S.H., } \\
\text { Fujita T. }\end{array}$ & $\begin{array}{c}\text { A novel approach for } \\
\text { evaluating the performance of } \\
\text { eco-industrial park pilot } \\
\text { projects }\end{array}$ & $\begin{array}{l}\text { Journal of Cleaner } \\
\text { Production }\end{array}$ & 2013 & 39 & & 50 & 59 & 10.1016/j.jclepro.2012.08.030 \\
\hline
\end{tabular}




\begin{tabular}{|c|c|c|c|c|c|c|c|c|}
\hline Author Name(s) & Title & Source Title & Year & Vol. & Issue & P. Start & P. End & Doi \\
\hline $\begin{array}{l}\text { Mohammed F.A., } \\
\text { Biswas W.K., } \\
\text { Yao H.M., Tadé } \\
\text { M.O. } \\
\end{array}$ & $\begin{array}{c}\text { Assessment of industrial by- } \\
\text { product synergies from process } \\
\text { engineering and sustainability } \\
\text { principles }\end{array}$ & $\begin{array}{l}\text { Progress in Industrial } \\
\text { Ecology }\end{array}$ & 2013 & 8 & 3 & 156 & 165 & 10.1504/PIE.2013.060663 \\
\hline $\begin{array}{l}\text { Romero E., Ruiz } \\
\text { M.C. }\end{array}$ & $\begin{array}{l}\text { Framework for applying a } \\
\text { complex adaptive system } \\
\text { approach to model the } \\
\text { operation of eco-industrial } \\
\text { parks } \\
\end{array}$ & $\begin{array}{l}\text { Journal of Industrial } \\
\text { Ecology }\end{array}$ & 2013 & 17 & 5 & 731 & 741 & 10.1111/jiec. 12032 \\
\hline $\begin{array}{c}\text { Patala S., } \\
\text { Hämäläinen S., } \\
\text { Jalkala A., } \\
\text { Pesonen H.-L. } \\
\end{array}$ & $\begin{array}{l}\text { Towards a broader perspective } \\
\text { on the forms of eco-industrial } \\
\text { networks }\end{array}$ & $\begin{array}{l}\text { Journal of Cleaner } \\
\text { Production }\end{array}$ & 2014 & 82 & & 166 & 178 & 10.1016/j.jclepro.2014.06.059 \\
\hline $\begin{array}{l}\text { Giurco D., Prior } \\
\text { J., Boydell S. }\end{array}$ & $\begin{array}{l}\text { Industrial ecology and carbon } \\
\text { property rights }\end{array}$ & $\begin{array}{c}\text { Journal of Cleaner } \\
\text { Production }\end{array}$ & 2014 & 80 & & 211 & 223 & 10.1016/j.jclepro.2014.05.079 \\
\hline $\begin{array}{l}\text { Short S.W., } \\
\text { Bocken N.M., } \\
\text { Barlow C.Y., } \\
\text { Chertow M.R. }\end{array}$ & $\begin{array}{l}\text { From refining sugar to growing } \\
\text { tomatoes: Industrial ecology } \\
\text { and business model evolution } \\
\text { short et al. from refining sugar } \\
\text { to growing tomatoes }\end{array}$ & $\begin{array}{l}\text { Journal of Industrial } \\
\text { Ecology }\end{array}$ & 2014 & & & & & 10.1111/jiec. 12171 \\
\hline $\begin{array}{l}\text { Yu B., Li X., Shi } \\
\text { L., Qian Y. }\end{array}$ & $\begin{array}{l}\text { Quantifying CO2 emission } \\
\text { reduction from industrial } \\
\text { symbiosis in integrated steel } \\
\text { mills in China }\end{array}$ & $\begin{array}{l}\text { Journal of Cleaner } \\
\text { Production }\end{array}$ & 2014 & & & & & 10.1016/j.jclepro.2014.08.015 \\
\hline $\begin{array}{l}\text { Chopra S.S., } \\
\text { Khanna V. }\end{array}$ & $\begin{array}{l}\text { Understanding resilience in } \\
\text { industrial symbiosis networks: } \\
\text { Insights from network analysis }\end{array}$ & $\begin{array}{c}\text { Journal of } \\
\text { Environmental } \\
\text { Management }\end{array}$ & 2014 & 141 & & 86 & 94 & 10.1016/j.jenvman.2013.12.038 \\
\hline $\begin{array}{l}\text { Schiller F., Penn } \\
\text { A.S., Basson L. }\end{array}$ & $\begin{array}{c}\text { Analyzing networks in } \\
\text { industrial ecology - A review } \\
\text { of Social-Material Network } \\
\text { Analyses } \\
\end{array}$ & $\begin{array}{l}\text { Journal of Cleaner } \\
\text { Production }\end{array}$ & 2014 & 76 & & 1 & 11 & 10.1016/j.jclepro.2014.03.029 \\
\hline $\begin{array}{l}\text { Li W., Cui Z., } \\
\text { Han F. }\end{array}$ & $\begin{array}{l}\text { Methods for assessing the } \\
\text { energy-saving efficiency of } \\
\text { industrial symbiosis in } \\
\text { industrial parks } \\
\end{array}$ & $\begin{array}{l}\text { Environmental Science } \\
\text { and Pollution Research }\end{array}$ & 2014 & & & & & $10.1007 / \mathrm{s} 11356-014-3327-4$ \\
\hline $\begin{array}{l}\text { Li W., Cui Z., } \\
\text { Han F. }\end{array}$ & $\begin{array}{l}\text { Methods for assessing the } \\
\text { energy-saving efficiency of } \\
\text { industrial symbiosis in } \\
\text { industrial parks }\end{array}$ & $\begin{array}{l}\text { Environmental Science } \\
\text { and Pollution Research }\end{array}$ & 2014 & 22 & 1 & 275 & 285 & $10.1007 / \mathrm{s} 11356-014-3327-4$ \\
\hline
\end{tabular}

APPLIED ECOLOGY AND ENVIRONMENTAL RESEARCH 17(1):1159-1221.

http://www.aloki.hu • ISSN 15891623 (Print) • ISSN 17850037 (Online)

DOI: http://dx.doi.org/10.15666/aeer/1701_11591221

(c) 2019, ALÖKI Kft., Budapest, Hungary 


\begin{tabular}{|c|c|c|c|c|c|c|c|c|}
\hline Author Name(s) & Title & Source Title & Year & Vol. & Issue & P. Start & P. End & Doi \\
\hline $\begin{array}{c}\text { Trokanas N., } \\
\text { Cecelja F., Raafat } \\
\text { T. }\end{array}$ & $\begin{array}{l}\text { Semantic input/output } \\
\text { matching for waste processing } \\
\text { in industrial symbiosis }\end{array}$ & $\begin{array}{l}\text { Computers and } \\
\text { Chemical Engineering }\end{array}$ & 2014 & 66 & & 259 & 268 & 10.1016/j.compchemeng.2014.02.010 \\
\hline $\begin{array}{l}\text { Cerceau J., Mat } \\
\text { N., Junqua G., } \\
\text { Lin L., Laforest } \\
\text { V., Gonzalez C. } \\
\end{array}$ & $\begin{array}{l}\text { Implementing industrial } \\
\text { ecology in port cities: } \\
\text { International overview of case } \\
\text { studies and cross-case analysis }\end{array}$ & $\begin{array}{l}\text { Journal of Cleaner } \\
\text { Production }\end{array}$ & 2014 & 74 & & 1 & 16 & 10.1016/j.jclepro.2014.03.050 \\
\hline Harmsen J. & $\begin{array}{l}\text { Novel sustainable industrial } \\
\text { processes: From idea to } \\
\text { commercial scale } \\
\text { implementation }\end{array}$ & $\begin{array}{l}\text { Green Processing and } \\
\text { Synthesis }\end{array}$ & 2014 & 3 & 3 & 189 & 193 & 10.1515/gps-2013-0102 \\
\hline Jiao W., Boons F. & $\begin{array}{l}\text { Toward a research agenda for } \\
\text { policy intervention and } \\
\text { facilitation to enhance } \\
\text { industrial symbiosis based on a } \\
\text { comprehensive literature } \\
\text { review }\end{array}$ & $\begin{array}{l}\text { Journal of Cleaner } \\
\text { Production }\end{array}$ & 2014 & 67 & & 14 & 25 & 10.1016/j.jclepro.2013.12.050 \\
\hline $\begin{array}{l}\text { Simboli A., } \\
\text { Taddeo R., } \\
\text { Morgante A. }\end{array}$ & $\begin{array}{l}\text { Analysing the development of } \\
\text { Industrial Symbiosis in a } \\
\text { motorcycle local industrial } \\
\text { network: The role of contextual } \\
\text { factors }\end{array}$ & $\begin{array}{l}\text { Journal of Cleaner } \\
\text { Production }\end{array}$ & 2014 & 66 & & 372 & 383 & 10.1016/j.jclepro.2013.11.045 \\
\hline Aviso K.B. & $\begin{array}{l}\text { Design of robust water } \\
\text { exchange networks for eco- } \\
\text { industrial symbiosis }\end{array}$ & $\begin{array}{l}\text { Process Safety and } \\
\text { Environmental } \\
\text { Protection }\end{array}$ & 2014 & 92 & 2 & 160 & 170 & 10.1016/j.psep.2012.12.001 \\
\hline Zhu J., Ruth M. & $\begin{array}{l}\text { The development of regional } \\
\text { collaboration for resource } \\
\text { efficiency: A network } \\
\text { perspective on industrial } \\
\text { symbiosis }\end{array}$ & $\begin{array}{l}\text { Computers, } \\
\text { Environment and Urban } \\
\text { Systems }\end{array}$ & 2014 & 44 & & 37 & 46 & 10.1016/j.compenvurbsys.2013.11.001 \\
\hline $\begin{array}{c}\text { Zhang B., Wang } \\
\text { Z. }\end{array}$ & $\begin{array}{l}\text { Inter-firm collaborations on } \\
\text { carbon emission reduction } \\
\text { within industrial chains in } \\
\text { China: Practices, drivers and } \\
\text { effects on firms' performances }\end{array}$ & Energy Economics & 2014 & 42 & & 115 & 131 & 10.1016/j.eneco.2013.12.006 \\
\hline $\begin{array}{l}\text { Mirabella N., } \\
\text { Castellani V., } \\
\text { Sala S. }\end{array}$ & $\begin{array}{l}\text { Current options for the } \\
\text { valorization of food } \\
\text { manufacturing waste: A review }\end{array}$ & $\begin{array}{l}\text { Journal of Cleaner } \\
\text { Production }\end{array}$ & 2014 & 65 & & 28 & 41 & 10.1016/j.jclepro.2013.10.051 \\
\hline
\end{tabular}




\begin{tabular}{|c|c|c|c|c|c|c|c|c|}
\hline Author Name(s) & $\begin{array}{c}\text { Title } \\
\end{array}$ & Source Title & Year & Vol. & Issue & P. Start & P. End & Doi \\
\hline $\begin{array}{c}\text { Gonela V., Zhang } \\
\text { J. }\end{array}$ & $\begin{array}{l}\text { Design of the optimal industrial } \\
\text { symbiosis system to improve } \\
\text { bioethanol production }\end{array}$ & $\begin{array}{l}\text { Journal of Cleaner } \\
\text { Production }\end{array}$ & 2014 & 64 & & 513 & 534 & 10.1016/j.jclepro.2013.07.059 \\
\hline $\begin{array}{l}\text { Dong L., Gu F., } \\
\text { Fujita T., Hayashi } \\
\text { Y., Gao J. }\end{array}$ & $\begin{array}{l}\text { Uncovering opportunity of } \\
\text { low-carbon city promotion with } \\
\text { industrial system innovation: } \\
\text { Case study on industrial } \\
\text { symbiosis projects in China }\end{array}$ & Energy Policy & 2014 & 65 & & 388 & 397 & 10.1016/j.enpol.2013.10.019 \\
\hline $\begin{array}{l}\text { Park H.-S., } \\
\text { Behera S.K. }\end{array}$ & $\begin{array}{l}\text { Methodological aspects of } \\
\text { applying eco-efficiency } \\
\text { indicators to industrial } \\
\text { symbiosis networks }\end{array}$ & $\begin{array}{l}\text { Journal of Cleaner } \\
\text { Production }\end{array}$ & 2014 & 64 & & 478 & 485 & 10.1016/j.jclepro.2013.08.032 \\
\hline $\begin{array}{l}\text { Yu C., De Jong } \\
\text { M., Dijkema } \\
\text { G.P.J. }\end{array}$ & $\begin{array}{c}\text { Process analysis of eco- } \\
\text { industrial park development - } \\
\text { The case of Tianjin, China }\end{array}$ & $\begin{array}{l}\text { Journal of Cleaner } \\
\text { Production }\end{array}$ & 2014 & 64 & & 464 & 477 & 10.1016/j.jclepro.2013.09.002 \\
\hline $\begin{array}{l}\text { Tian J., Liu W., } \\
\text { Lai B., Li X., } \\
\text { Chen L. }\end{array}$ & $\begin{array}{c}\text { Study of the performance of } \\
\text { eco-industrial park } \\
\text { development in China }\end{array}$ & $\begin{array}{l}\text { Journal of Cleaner } \\
\text { Production }\end{array}$ & 2014 & 64 & & 486 & 494 & 10.1016/j.jclepro.2013.08.005 \\
\hline $\begin{array}{l}\text { Liu G.F., Ma } \\
\text { Y.T. }\end{array}$ & $\begin{array}{c}\text { Study on by-product synergy } \\
\text { and eco-industrial parks in } \\
\text { China based on american } \\
\text { experience }\end{array}$ & $\begin{array}{c}\text { Applied Mechanics and } \\
\text { Materials }\end{array}$ & 2014 & 472 & & 884 & 888 & 10.4028/www.scientific.net/AMM.472.884 \\
\hline $\begin{array}{c}\text { Romero E., Ruiz } \\
\text { M.C. }\end{array}$ & $\begin{array}{c}\text { Proposal of an agent-based } \\
\text { analytical model to convert } \\
\text { industrial areas in industrial } \\
\text { eco-systems }\end{array}$ & $\begin{array}{l}\text { Science of the Total } \\
\text { Environment }\end{array}$ & 2014 & $\begin{array}{l}468- \\
469\end{array}$ & & 394 & 405 & 10.1016/j.scitotenv.2013.08.049 \\
\hline $\begin{array}{l}\text { Notarnicola B., } \\
\text { Tassielli G., } \\
\text { Renzulli P.A. }\end{array}$ & $\begin{array}{l}\text { Potential developments of } \\
\text { industrial symbiosis in the } \\
\text { Taranto productive district }\end{array}$ & $\begin{array}{l}\text { Pathways to } \\
\text { Environmental } \\
\text { Sustainability: } \\
\text { Methodologies and } \\
\text { Experiences }\end{array}$ & 2014 & & & 215 & 224 & 10.1007/978-3-319-03826-1_21 \\
\hline $\begin{array}{l}\text { Teh B.T., Ho } \\
\text { C.S., Matsuoka } \\
\text { Y., Chau L.W., } \\
\text { Gomi K. } \\
\end{array}$ & $\begin{array}{l}\text { Determinant factors of } \\
\text { industrial symbiosis: Greening } \\
\text { Pasir Gudang industrial park }\end{array}$ & $\begin{array}{l}\text { IOP Conference Series: } \\
\quad \text { Earth and } \\
\text { Environmental Science }\end{array}$ & 2014 & 18 & 1 & & & $10.1088 / 1755-1315 / 18 / 1 / 012162$ \\
\hline
\end{tabular}

APPLIED ECOLOGY AND ENVIRONMENTAL RESEARCH 17(1):1159-1221.

http://www.aloki.hu • ISSN 15891623 (Print) • ISSN 17850037 (Online)

DOI: http://dx.doi.org/10.15666/aeer/1701_11591221

(c) 2019, ALÖKI Kft., Budapest, Hungary 


\begin{tabular}{|c|c|c|c|c|c|c|c|c|}
\hline Author Name(s) & Title & Source Title & Year & Vol. & Issue & P. Start & P. End & Doi \\
\hline $\begin{array}{c}\text { Park J.Y., Park } \\
\text { H.-S. }\end{array}$ & $\begin{array}{l}\text { Securing a competitive } \\
\text { advantage through industrial } \\
\text { symbiosis development: The } \\
\text { case of steam networking } \\
\text { practices in Ulsan park and } \\
\text { park competitive advantage and } \\
\text { industrial symbiosis }\end{array}$ & $\begin{array}{l}\text { Journal of Industrial } \\
\text { Ecology }\end{array}$ & 2014 & 18 & 5 & 677 & 683 & 10.1111/jiec. 12158 \\
\hline Deutz P. & $\begin{array}{l}\text { Food for thought: Seeking the } \\
\text { essence of industrial symbiosis }\end{array}$ & $\begin{array}{c}\text { Pathways to } \\
\text { Environmental } \\
\text { Sustainability: } \\
\text { Methodologies and } \\
\text { Experiences } \\
\end{array}$ & 2014 & & & 3 & 11 & 10.1007/978-3-319-03826-1_1 \\
\hline $\begin{array}{l}\text { Trokanas N., } \\
\text { Cecelja F., Yu } \\
\text { M., Raafat T. }\end{array}$ & $\begin{array}{l}\text { Optimising environmental } \\
\text { performance of symbiotic } \\
\text { networks using semantics }\end{array}$ & $\begin{array}{c}\text { Computer Aided } \\
\text { Chemical Engineering }\end{array}$ & 2014 & 33 & & 847 & 852 & 10.1016/B978-0-444-63456-6.50142-3 \\
\hline $\begin{array}{l}\text { Zhang Y., Zheng } \\
\text { H., Chen B., Su } \\
\text { M., Liu G. }\end{array}$ & $\begin{array}{c}\text { A review of industrial } \\
\text { symbiosis research: theory and } \\
\text { methodology }\end{array}$ & $\begin{array}{l}\text { Frontiers of Earth } \\
\text { Science }\end{array}$ & 2014 & 9 & 1 & 91 & 104 & $10.1007 / \mathrm{s} 11707-014-0445-8$ \\
\hline $\begin{array}{l}\text { Geng Y., Liu Z., } \\
\text { Xue B., Dong H., } \\
\text { Fujita T., Chiu A. }\end{array}$ & $\begin{array}{c}\text { Emergy-based assessment on } \\
\text { industrial symbiosis: a case of } \\
\text { Shenyang Economic and } \\
\text { Technological Development } \\
\text { Zone } \\
\end{array}$ & $\begin{array}{l}\text { Environmental Science } \\
\text { and Pollution Research }\end{array}$ & 2014 & 21 & 23 & 13572 & 13587 & $10.1007 / \mathrm{s} 11356-014-3287-8$ \\
\hline $\begin{array}{l}\text { Paquin R.L., } \\
\text { Tilleman S.G., } \\
\text { Howard- } \\
\text { Grenville J. } \\
\end{array}$ & $\begin{array}{l}\text { Is there cash in that trash?: } \\
\text { Factors influencing industrial } \\
\text { symbiosis exchange initiation } \\
\text { and completion }\end{array}$ & $\begin{array}{c}\text { Journal of Industrial } \\
\text { Ecology }\end{array}$ & 2014 & 18 & 2 & 268 & 279 & 10.1111/jiec. 12120 \\
\hline $\begin{array}{l}\text { Xu S., Liu G., Lv } \\
\text { W., Liu Y. }\end{array}$ & $\begin{array}{l}\text { The nonlinear complementarity } \\
\text { model of industrial symbiosis } \\
\text { network equilibrium problem }\end{array}$ & $\begin{array}{l}\text { RAIRO - Operations } \\
\text { Research }\end{array}$ & 2014 & 48 & 4 & 559 & 594 & $10.1051 / \mathrm{ro} / 2014024$ \\
\hline $\begin{array}{l}\text { Boons F., } \\
\text { Spekkink W., } \\
\text { Jiao W. }\end{array}$ & $\begin{array}{c}\text { A Process Perspective on } \\
\text { Industrial Symbiosis: Theory, } \\
\text { Methodology, and Application } \\
\text { Boons et al. A Process } \\
\text { Perspective on Industrial } \\
\text { Symbiosis }\end{array}$ & $\begin{array}{l}\text { Journal of Industrial } \\
\text { Ecology }\end{array}$ & 2014 & 18 & 3 & 341 & 355 & 10.1111/jiec.12116 \\
\hline $\begin{array}{c}\text { Trokanas N., } \\
\text { Cecelja F., Raafat } \\
\text { T. }\end{array}$ & $\begin{array}{l}\text { Towards a re-usable ontology } \\
\text { for waste processing }\end{array}$ & $\begin{array}{c}\text { Computer Aided } \\
\text { Chemical Engineering }\end{array}$ & 2014 & 33 & & 841 & 846 & 10.1016/B978-0-444-63456-6.50141-1 \\
\hline
\end{tabular}

APPLIED ECOLOGY AND ENVIRONMENTAL RESEARCH 17(1):1159-1221.

http://www.aloki.hu • ISSN 15891623 (Print) • ISSN 17850037 (Online)

DOI: http://dx.doi.org/10.15666/aeer/1701_11591221

(c) 2019, ALÖKI Kft., Budapest, Hungary 


\begin{tabular}{|c|c|c|c|c|c|c|c|c|}
\hline Author Name(s) & Title & Source Title & Year & Vol. & Issue & P. Start & P. End & Doi \\
\hline $\begin{array}{c}\text { Cutaia L., } \\
\text { Morabito R., } \\
\text { Barberio G., } \\
\text { Mancuso E., } \\
\text { Brunori C., } \\
\text { Spezzano P., } \\
\text { Mione A., } \\
\text { Mungiguerra C., } \\
\text { Li Rosi O., } \\
\text { Cappello F. } \\
\end{array}$ & $\begin{array}{l}\text { The project for the } \\
\text { implementation of the } \\
\text { industrial symbiosis platform in } \\
\text { sicily: The progress after the } \\
\text { first year of operation }\end{array}$ & $\begin{array}{l}\text { Pathways to } \\
\text { Environmental } \\
\text { Sustainability: } \\
\text { Methodologies and } \\
\text { Experiences }\end{array}$ & 2014 & & & 205 & 214 & 10.1007/978-3-319-03826-1_20 \\
\hline $\begin{array}{l}\text { Golev A., Corder } \\
\text { G.D., Giurco D.P. }\end{array}$ & $\begin{array}{l}\text { Industrial symbiosis in } \\
\text { gladstone: A decade of } \\
\text { progress and future } \\
\text { development }\end{array}$ & $\begin{array}{l}\text { Journal of Cleaner } \\
\text { Production }\end{array}$ & 2014 & 84 & 1 & 421 & 429 & 10.1016/j.jclepro.2013.06.054 \\
\hline $\begin{array}{l}\text { Ng R.T.L., Ng } \\
\text { D.K.S., Tan R.R., } \\
\text { El-Halwagi M.M. }\end{array}$ & $\begin{array}{c}\text { Disjunctive fuzzy optimisation } \\
\text { for planning and synthesis of } \\
\text { bioenergy-based industrial } \\
\text { symbiosis system } \\
\end{array}$ & $\begin{array}{c}\text { Journal of } \\
\text { Environmental } \\
\text { Chemical Engineering }\end{array}$ & 2014 & 2 & 1 & 652 & 664 & 10.1016/j.jece.2013.11.003 \\
\hline $\begin{array}{l}\text { Schiller F., Penn } \\
\text { A., Druckman A., } \\
\text { Basson L., } \\
\text { Royston K. }\end{array}$ & $\begin{array}{c}\text { Exploring Space, Exploiting } \\
\text { Opportunities: The Case for } \\
\text { Analyzing Space in Industrial } \\
\text { Ecology Schiller et al. } \\
\text { Exploring Space, Exploiting } \\
\text { Opportunities }\end{array}$ & $\begin{array}{l}\text { Journal of Industrial } \\
\text { Ecology }\end{array}$ & 2014 & 18 & 6 & 792 & 798 & 10.1111/jiec. 12140 \\
\hline $\begin{array}{l}\text { Yu C., Davis C., } \\
\text { Dijkema G.P.J. }\end{array}$ & $\begin{array}{l}\text { Understanding the evolution of } \\
\text { industrial symbiosis research: } \\
\text { A bibliometric and network } \\
\text { analysis (1997-2012) }\end{array}$ & $\begin{array}{l}\text { Journal of Industrial } \\
\text { Ecology }\end{array}$ & 2014 & 18 & 2 & 280 & 293 & 10.1111/jiec. 12073 \\
\hline $\begin{array}{c}\text { Alfaro J., Miller } \\
\text { S. }\end{array}$ & $\begin{array}{c}\text { Applying Industrial Symbiosis } \\
\text { to Smallholder Farms: } \\
\text { Modeling a Case Study in } \\
\text { Liberia, West Africa }\end{array}$ & $\begin{array}{l}\text { Journal of Industrial } \\
\text { Ecology }\end{array}$ & 2014 & 18 & 1 & 145 & 154 & 10.1111/jiec.12077 \\
\hline $\begin{array}{l}\text { Zou Y.-L., Li C.- } \\
\text { F., Yao Z.-D., } \\
\text { Cao Y.-Y. } \\
\end{array}$ & $\begin{array}{c}\text { AN research on energy } \\
\text { management and cooperation } \\
\text { performance in eco-industrial } \\
\text { symbiosis network } \\
\end{array}$ & $\begin{array}{c}\text { Advanced Materials } \\
\text { Research }\end{array}$ & 2014 & $\begin{array}{l}986- \\
987\end{array}$ & & 211 & 214 & $\begin{array}{c}\text { 10.4028/www.scientific.net/AMR.986- } \\
987.211\end{array}$ \\
\hline
\end{tabular}




\begin{tabular}{|c|c|c|c|c|c|c|c|c|}
\hline Author Name(s) & Title & Source Title & Year & Vol. & Issue & P. Start & P. End & Doi \\
\hline $\begin{array}{l}\text { Ng R.T.L., } \\
\text { Hassim M.H., Ng } \\
\text { D.K.S., Tan R.R., } \\
\text { El-Halwagi M.M. }\end{array}$ & $\begin{array}{c}\text { Multi-objective design of } \\
\text { industrial symbiosis in palm oil } \\
\text { industry }\end{array}$ & $\begin{array}{l}\text { Computer Aided } \\
\text { Chemical Engineering }\end{array}$ & 2014 & 34 & & 579 & 584 & 10.1016/B978-0-444-63433-7.50081-X \\
\hline Li C., Feng L. & $\begin{array}{l}\text { Evolutionary game analysis of } \\
\text { the eco-industrial symbiosis } \\
\text { network considering externality }\end{array}$ & $\begin{array}{l}\text { Complex Systems and } \\
\text { Complexity Science }\end{array}$ & 2014 & 11 & 3 & 58 & 64 & 10.13306/j.1672-3813.2014.03.009 \\
\hline $\begin{array}{l}\text { Ng R.T.L., Wan } \\
\text { Y.K., Ng D.K.S., } \\
\text { Tan R.R. }\end{array}$ & $\begin{array}{l}\text { Stability analysis of symbiotic } \\
\text { bioenergy parks }\end{array}$ & $\begin{array}{l}\text { Chemical Engineering } \\
\text { Transactions }\end{array}$ & 2014 & 39 & & 859 & 864 & 10.3303/CET1439144 \\
\hline $\begin{array}{l}\text { Noureldin M.B., } \\
\text { Farooq Z., Al- } \\
\text { Owaidh M., Al- } \\
\text { Saed H. }\end{array}$ & $\begin{array}{l}\text { New systematic approach using } \\
\text { combined constraints logic } \\
\text { propagation and mathematical } \\
\text { programming techniques for } \\
\text { energy efficient synthesis of } \\
\text { eco-industrial parks }\end{array}$ & $\begin{array}{l}\text { Process Development } \\
\text { Division } 2014 \text { - Core } \\
\text { Programming Area at } \\
\text { the 2014 AIChE Annual } \\
\text { Meeting }\end{array}$ & 2014 & & & 305 & 307 & \\
\hline $\begin{array}{c}\text { Benjamin } \\
\text { M.F.D., Tan } \\
\text { R.R., Razon L.F. }\end{array}$ & $\begin{array}{c}\text { A methodology for criticality } \\
\text { analysis in symbiotic bioenergy } \\
\text { parks }\end{array}$ & Energy Procedia & 2014 & 61 & & 41 & 44 & 10.1016/j.egypro.2014.11.901 \\
\hline $\begin{array}{l}\text { Ramli A., } \\
\text { Mokhtar M., Aziz } \\
\text { B.A., Ngah N.A. }\end{array}$ & $\begin{array}{l}\text { The cooperative approach in } \\
\text { managing safety issues for } \\
\text { Halal industrial parks in } \\
\text { Malaysia: Embracing } \\
\text { opportunity }\end{array}$ & $\begin{array}{c}\text { Progress in Industrial } \\
\text { Ecology }\end{array}$ & 2014 & 8 & 4 & 295 & 318 & 10.1504/PIE.2014.066805 \\
\hline $\begin{array}{c}\text { Carmen Lenuta } \\
\text { T. }\end{array}$ & $\begin{array}{l}\text { Contributions to the fondation } \\
\text { of a waste management plan on } \\
\text { an area level }\end{array}$ & $\begin{array}{c}\text { International } \\
\text { Multidisciplinary } \\
\text { Scientific } \\
\text { GeoConference } \\
\text { Surveying Geology and } \\
\text { Mining Ecology } \\
\text { Management, SGEM }\end{array}$ & 2014 & 3 & 5 & 95 & 101 & \\
\hline $\begin{array}{l}\text { Short S.W., } \\
\text { Bocken N.M.P., } \\
\text { Barlow C.Y., } \\
\text { Chertow M.R. } \\
\end{array}$ & $\begin{array}{l}\text { From refining sugar to growing } \\
\text { tomatoes: Industrial ecology } \\
\text { and business model evolution }\end{array}$ & $\begin{array}{l}\text { Journal of Industrial } \\
\text { Ecology }\end{array}$ & 2014 & 18 & 5 & 603 & 618 & 10.1111/jiec.12171 \\
\hline
\end{tabular}




\begin{tabular}{|c|c|c|c|c|c|c|c|c|}
\hline Author Name(s) & Title & Source Title & Year & Vol. & Issue & P. Start & P. End & Doi \\
\hline $\begin{array}{l}\text { Arranz P., Anzizu } \\
\text { M., Pineau A., } \\
\text { Marwede M., } \\
\text { Den Boer E., Den } \\
\text { Boer J., } \\
\text { Cocciantelli J.- } \\
\text { M., Williams } \\
\text { I.D., Obersteiner } \\
\text { G., Scherhaufer } \\
\text { S., Vallvé X. }\end{array}$ & $\begin{array}{l}\text { The development of a } \\
\text { resourceefficient photovoltaic } \\
\text { system }\end{array}$ & $\begin{array}{c}\text { Proceedings of } \\
\text { Institution of Civil } \\
\text { Engineers: Waste and } \\
\text { Resource Management }\end{array}$ & 2014 & 167 & 3 & 109 & 122 & 10.1680/warm.13.00027 \\
\hline $\begin{array}{c}\text { Steingrimsson } \\
\text { J.G., Seliger G. }\end{array}$ & $\begin{array}{c}\text { Conceptual framework for } \\
\text { near-to-site waste cycle design }\end{array}$ & Procedia CIRP & 2014 & 15 & & 272 & 277 & 10.1016/j.procir.2014.06.014 \\
\hline $\begin{array}{l}\text { Obersteiner G., } \\
\text { Pertl A. }\end{array}$ & $\begin{array}{l}\text { Waste Avoidance Through } \\
\text { Industrial Symbiosis } \\
\text { [Abfallvermeidung durch } \\
\text { industrielle Symbiose] }\end{array}$ & $\begin{array}{l}\text { Osterreichische Wasser- } \\
\text { und Abfallwirtschaft }\end{array}$ & 2014 & 66 & & 417 & 423 & 10.1007/s00506-014-0191-x \\
\hline $\begin{array}{l}\text { Dong H., Ohnishi } \\
\text { S., Fujita T., } \\
\text { Geng Y., Fujii } \\
\text { M., Dong L. } \\
\end{array}$ & $\begin{array}{c}\text { Achieving carbon emission } \\
\text { reduction through industrial \& } \\
\text { urban symbiosis: A case of } \\
\text { Kawasaki }\end{array}$ & Energy & 2014 & 64 & & 277 & 286 & 10.1016/j.energy.2013.11.005 \\
\hline $\begin{array}{l}\text { Noureldin M.B., } \\
\text { Farooq Z., Al- } \\
\text { Owaidh M., Al- } \\
\text { Saed H. }\end{array}$ & $\begin{array}{l}\text { New systematic approach using } \\
\text { combined constraints logic } \\
\text { propagation and mathematical } \\
\text { programming techniques for } \\
\text { energy efficient synthesis of } \\
\text { eco-industrial parks }\end{array}$ & $\begin{array}{l}\text { Sustainable Engineering } \\
\text { Forum } 2014 \text { - Core } \\
\text { Programming Area at } \\
\text { the 2014 AIChE Annual } \\
\text { Meeting }\end{array}$ & 2014 & & & 351 & 353 & \\
\hline $\begin{array}{l}\text { Li X., Xiao R., } \\
\text { Zeng Y., Yao Z. }\end{array}$ & $\begin{array}{l}\text { Vulnerability analysis of } \\
\text { symbiotic network in eco- } \\
\text { industrial parks }\end{array}$ & $\begin{array}{l}\text { Shengtai Xuebao/ Acta } \\
\text { Ecologica Sinica }\end{array}$ & 2014 & 34 & 16 & 4746 & 4755 & $10.5846 / \mathrm{stxb} 201212191820$ \\
\hline $\begin{array}{l}\text { Eckelman M.J., } \\
\text { Ashton W., } \\
\text { Arakaki Y., } \\
\text { Hanaki K., } \\
\text { Nagashima S., } \\
\text { Malone-Lee L.C. }\end{array}$ & $\begin{array}{c}\text { Island waste management } \\
\text { systems: Statistics, challenges, } \\
\text { and opportunities for applied } \\
\text { industrial ecology }\end{array}$ & $\begin{array}{c}\text { Journal of Industrial } \\
\text { Ecology }\end{array}$ & 2014 & 18 & 2 & 306 & 317 & 10.1111/jiec.12113 \\
\hline Stubbs W. & $\begin{array}{c}\text { Exploration of barriers to } \\
\text { mainstreaming industrial } \\
\text { ecosystems in Australia }\end{array}$ & $\begin{array}{l}\text { Progress in Industrial } \\
\text { Ecology }\end{array}$ & 2014 & 8 & 4 & 319 & 335 & 10.1504/PIE.2014.066814 \\
\hline
\end{tabular}




\begin{tabular}{|c|c|c|c|c|c|c|c|c|}
\hline Author Name(s) & Title & Source Title & Year & Vol. & Issue & P. Start & P. End & Doi \\
\hline $\begin{array}{l}\text { Rosano M., } \\
\text { Schianetz K. }\end{array}$ & $\begin{array}{l}\text { Measuring sustainability } \\
\text { performance in industrial } \\
\text { parks: A case study of the } \\
\text { Kwinana industrial area }\end{array}$ & $\begin{array}{c}\text { International Journal of } \\
\text { Sustainable } \\
\text { Development }\end{array}$ & 2014 & 17 & 3 & 261 & 280 & 10.1504/IJSD.2014.064181 \\
\hline $\begin{array}{l}\text { Wang G., Feng } \\
\text { X., Khim Hoong } \\
\text { C. }\end{array}$ & $\begin{array}{l}\text { Symbiosis analysis on } \\
\text { industrial ecological system }\end{array}$ & $\begin{array}{l}\text { Chinese Journal of } \\
\text { Chemical Engineering }\end{array}$ & 2014 & 22 & 6 & 690 & 698 & $10.1016 / S 1004-9541(14) 60084-7$ \\
\hline $\begin{array}{l}\text { Dean C.A., Fath } \\
\text { B.D., Chen B. }\end{array}$ & $\begin{array}{c}\text { Indicators for an expanded } \\
\text { business operations model to } \\
\text { evaluate eco-smart corporate } \\
\text { communities }\end{array}$ & Ecological Indicators & 2014 & 47 & & 137 & 148 & 10.1016/j.ecolind.2014.07.010 \\
\hline $\begin{array}{l}\text { Campos T.R.T., } \\
\text { Fonseca M.V.A., } \\
\text { Morais R.M.N. } \\
\end{array}$ & $\begin{array}{l}\text { Reverse logistics: A route that } \\
\text { only makes sense when } \\
\text { adopting a systemic vision }\end{array}$ & $\begin{array}{l}\text { WIT Transactions on } \\
\text { Ecology and the } \\
\text { Environment } \\
\end{array}$ & 2014 & 180 & & 41 & 52 & 10.2495/WM140041 \\
\hline $\begin{array}{l}\text { Kikuchi Y., } \\
\text { Kimura S., } \\
\text { Okamoto Y., } \\
\text { Koyama M. }\end{array}$ & $\begin{array}{l}\text { A scenario analysis of future } \\
\text { energy systems based on an } \\
\text { energy flow model represented } \\
\text { as functionals of technology } \\
\text { options }\end{array}$ & Applied Energy & 2014 & 132 & & 586 & 601 & 10.1016/j.apenergy.2014.07.005 \\
\hline Spekkink W. & $\begin{array}{l}\text { Building capacity for } \\
\text { sustainable regional industrial } \\
\text { systems: An event sequence } \\
\text { analysis of developments in the } \\
\text { Sloe Area and Canal Zone }\end{array}$ & $\begin{array}{l}\text { Journal of Cleaner } \\
\text { Production }\end{array}$ & 2014 & & & & & 10.1016/j.jclepro.2014.08.028 \\
\hline $\begin{array}{c}\text { Cecelja F., } \\
\text { Trokanas N., } \\
\text { Raafat T., Yu M. }\end{array}$ & $\begin{array}{c}\text { Semantic algorithm for } \\
\text { Industrial Symbiosis network } \\
\text { synthesis }\end{array}$ & $\begin{array}{c}\text { Computers and } \\
\text { Chemical Engineering }\end{array}$ & 2015 & 83 & & 248 & 266 & 10.1016/j.compchemeng.2015.04.031 \\
\hline $\begin{array}{c}\text { Chen P.-C., Ma } \\
\text { H.-W. }\end{array}$ & $\begin{array}{l}\text { Using an Industrial Waste } \\
\text { Account to Facilitate National } \\
\text { Level Industrial Symbioses by } \\
\text { Uncovering the Waste } \\
\text { Exchange Potential }\end{array}$ & $\begin{array}{l}\text { Journal of Industrial } \\
\text { Ecology }\end{array}$ & 2015 & 19 & 6 & 950 & 962 & 10.1111/jiec.12236 \\
\hline $\begin{array}{l}\text { Meylan F.D., } \\
\text { Moreau V., } \\
\text { Erkman S. }\end{array}$ & $\begin{array}{l}\mathrm{CO} 2 \text { utilization in the } \\
\text { perspective of industrial } \\
\text { ecology, an overview }\end{array}$ & $\begin{array}{c}\text { Journal of CO2 } \\
\text { Utilization }\end{array}$ & 2015 & 12 & & 101 & 108 & 10.1016/j.jcou.2015.05.003 \\
\hline $\begin{array}{l}\text { Patrício J., Costa } \\
\text { I., Niza S. }\end{array}$ & $\begin{array}{l}\text { Urban material cycle closing - } \\
\text { Assessment of industrial waste } \\
\text { management in Lisbon region }\end{array}$ & $\begin{array}{l}\text { Journal of Cleaner } \\
\text { Production }\end{array}$ & 2015 & 106 & & 389 & 399 & 10.1016/j.jclepro.2014.08.069 \\
\hline
\end{tabular}




\begin{tabular}{|c|c|c|c|c|c|c|c|c|}
\hline Author Name(s) & $\begin{array}{c}\text { Title } \\
\end{array}$ & Source Title & Year & Vol. & Issue & P. Start & P. End & Doi \\
\hline $\begin{array}{l}\text { Kwon G.-R., } \\
\text { Woo S.H., Lim } \\
\text { S.-R. }\end{array}$ & $\begin{array}{c}\text { Industrial ecology-based } \\
\text { strategies to reduce the } \\
\text { embodied } \mathrm{CO} 2 \text { of magnesium } \\
\text { metal }\end{array}$ & $\begin{array}{l}\text { Resources, } \\
\text { Conservation and } \\
\text { Recycling }\end{array}$ & 2015 & 104 & & 206 & 212 & 10.1016/j.resconrec.2015.08.008 \\
\hline Spekkink W. & $\begin{array}{l}\text { Varieties of industrial } \\
\text { symbiosis }\end{array}$ & $\begin{array}{c}\text { International } \\
\text { Perspectives on } \\
\text { Industrial Ecology }\end{array}$ & 2015 & & & 142 & 156 & $10.4337 / 9781781003572.00017$ \\
\hline Patchell J. & $\begin{array}{c}\text { Intersection of industrial } \\
\text { symbiosis and product-based } \\
\text { industrial ecologies: } \\
\text { Considerations from the } \\
\text { Japanese home appliance } \\
\text { industry }\end{array}$ & $\begin{array}{l}\text { International } \\
\text { Perspectives on } \\
\text { Industrial Ecology }\end{array}$ & 2015 & & & 175 & 190 & $10.4337 / 9781781003572.00019$ \\
\hline $\begin{array}{l}\text { Wang Q., Deutz } \\
\text { P., Gibbs D. }\end{array}$ & $\begin{array}{l}\text { UK-China collaboration for } \\
\text { industrial symbiosis: A multi- } \\
\text { level approach to policy } \\
\text { transfer analysis }\end{array}$ & $\begin{array}{l}\text { International } \\
\text { Perspectives on } \\
\text { Industrial Ecology }\end{array}$ & 2015 & & & 89 & 107 & $10.4337 / 9781781003572.00014$ \\
\hline Olayide O.E. & $\begin{array}{l}\text { Industrial ecology, industrial } \\
\text { symbiosis and eco-industrial } \\
\text { parks in Africa: Issues for } \\
\text { sustainable development }\end{array}$ & $\begin{array}{l}\text { International } \\
\text { Perspectives on } \\
\text { Industrial Ecology }\end{array}$ & 2015 & & & 30 & 45 & $10.4337 / 9781781003572.00011$ \\
\hline $\begin{array}{c}\text { Boons F., } \\
\text { Spekkink W., } \\
\text { Isenmann R., } \\
\text { Baas L., Eklund } \\
\text { M., Brullot S., } \\
\text { Deutz P., Gibbs } \\
\text { D., Massard G., } \\
\text { Arozamena E.R., } \\
\text { Puente C.R., } \\
\text { Verguts V., Davis } \\
\text { C., Korevaar G., } \\
\text { Costa I., } \\
\text { Baumann H. }\end{array}$ & $\begin{array}{l}\text { Comparing industrial } \\
\text { symbiosis in Europe: Towards } \\
\text { a conceptual framework and } \\
\text { research methodology }\end{array}$ & $\begin{array}{l}\text { International } \\
\text { Perspectives on } \\
\text { Industrial Ecology }\end{array}$ & 2015 & & & 69 & 88 & $10.4337 / 9781781003572.00013$ \\
\hline $\begin{array}{l}\text { Deutz P., Lyons } \\
\text { D.I., Bi J. }\end{array}$ & $\begin{array}{l}\text { International perspectives on } \\
\text { industrial ecology }\end{array}$ & $\begin{array}{c}\text { International } \\
\text { Perspectives on } \\
\text { Industrial Ecology } \\
\end{array}$ & 2015 & & & 1 & 249 & $10.4337 / 9781781003572$ \\
\hline
\end{tabular}

APPLIED ECOLOGY AND ENVIRONMENTAL RESEARCH 17(1):1159-1221.

http://www.aloki.hu • ISSN 15891623 (Print) • ISSN 17850037 (Online)

DOI: http://dx.doi.org/10.15666/aeer/1701_11591221

๑ 2019, ALÖKI Kft., Budapest, Hungary 


\begin{tabular}{|c|c|c|c|c|c|c|c|c|}
\hline Author Name(s) & Title & Source Title & Year & Vol. & Issue & P. Start & P. End & Doi \\
\hline $\begin{array}{l}\text { Iacondini A., } \\
\text { Mencherini U., } \\
\text { Passarini F., } \\
\text { Vassura I., } \\
\text { Fanelli A., Cibotti } \\
\text { P. } \\
\end{array}$ & $\begin{array}{c}\text { Feasibility of Industrial } \\
\text { Symbiosis in Italy as an } \\
\text { Opportunity for Economic } \\
\text { Development: Critical Success } \\
\text { Factor Analysis, Impact and } \\
\text { Constrains of the Specific } \\
\text { Italian Regulations } \\
\end{array}$ & $\begin{array}{c}\text { Waste and Biomass } \\
\text { Valorization }\end{array}$ & 2015 & 6 & 5 & 865 & 874 & $10.1007 / \mathrm{s} 12649-015-9380-5$ \\
\hline $\begin{array}{c}\text { Madsen J.K., } \\
\text { Boisen N., } \\
\text { Nielsen L.U., } \\
\text { Tackmann L.H. }\end{array}$ & $\begin{array}{l}\text { Industrial Symbiosis } \\
\text { Exchanges: Developing a } \\
\text { Guideline to Companies }\end{array}$ & $\begin{array}{c}\text { Waste and Biomass } \\
\text { Valorization }\end{array}$ & 2015 & 6 & 5 & 855 & 864 & $10.1007 / s 12649-015-9417-9$ \\
\hline $\begin{array}{l}\text { Zoccola M., } \\
\text { Montarsolo A., } \\
\text { Mossotti R., } \\
\text { Patrucco A., } \\
\text { Tonin C. }\end{array}$ & $\begin{array}{l}\text { Green Hydrolysis as an } \\
\text { Emerging Technology to Turn } \\
\text { Wool Waste into Organic } \\
\text { Nitrogen Fertilizer }\end{array}$ & $\begin{array}{c}\text { Waste and Biomass } \\
\text { Valorization }\end{array}$ & 2015 & 6 & 5 & 891 & 897 & $10.1007 / \mathrm{s} 12649-015-9393-0$ \\
\hline $\begin{array}{l}\text { Lombardi L., } \\
\text { Carnevale E., } \\
\text { Baciocchi R., } \\
\text { Costa G. }\end{array}$ & $\begin{array}{c}\text { Biogas Upgrading by a } \\
\text { Combination of Innovative } \\
\text { Treatments Based on } \\
\text { Carbonation of Waste } \\
\text { Incineration Residues } \\
\end{array}$ & $\begin{array}{c}\text { Waste and Biomass } \\
\text { Valorization }\end{array}$ & 2015 & 6 & 5 & 791 & 803 & $10.1007 / \mathrm{s} 12649-015-9413-0$ \\
\hline Earley K. & $\begin{array}{c}\text { Industrial symbiosis: } \\
\text { Harnessing waste energy and } \\
\text { materials for mutual benefit }\end{array}$ & $\begin{array}{l}\text { Renewable Energy } \\
\text { Focus }\end{array}$ & 2015 & 16 & 4 & 75 & 77 & 10.1016/j.ref.2015.09.011 \\
\hline $\begin{array}{c}\text { Atkins M.J., } \\
\text { Walmsley } \\
\text { M.R.W., } \\
\text { Walmsley T.G., } \\
\text { Neale J.R. }\end{array}$ & $\begin{array}{l}\text { Integration of biomass } \\
\text { conversion technologies and } \\
\text { geothermal heat into a model } \\
\text { wood processing cluster }\end{array}$ & $\begin{array}{c}\text { Chemical Engineering } \\
\text { Transactions }\end{array}$ & 2015 & 45 & & 169 & 174 & 10.3303/CET1545029 \\
\hline $\begin{array}{l}\text { Gonela V., Zhang } \\
\text { J., Osmani A. }\end{array}$ & $\begin{array}{c}\text { Stochastic optimization of } \\
\text { sustainable industrial symbiosis } \\
\text { based hybrid generation } \\
\text { bioethanol supply chains }\end{array}$ & $\begin{array}{c}\text { Computers and } \\
\text { Industrial Engineering }\end{array}$ & 2015 & 87 & & 40 & 65 & 10.1016/j.cie.2015.04.025 \\
\hline $\begin{array}{l}\text { Abate S., } \\
\text { Lanzafame P., } \\
\text { Perathoner S., } \\
\text { Centi G. }\end{array}$ & $\begin{array}{l}\text { New Sustainable Model of } \\
\text { Biorefineries: Biofactories and } \\
\text { Challenges of Integrating Bio- } \\
\text { and Solar Refineries }\end{array}$ & ChemSusChem & 2015 & 8 & 17 & 2854 & 2866 & $10.1002 /$ cssc. 201500277 \\
\hline
\end{tabular}




\begin{tabular}{|c|c|c|c|c|c|c|c|c|}
\hline Author Name(s) & Title & Source Title & Year & Vol. & Issue & P. Start & P. End & Doi \\
\hline $\begin{array}{l}\text { Benjamin } \\
\text { M.F.D., Ubando } \\
\text { A.T., Razon L.F., } \\
\text { Tan R.R. } \\
\end{array}$ & $\begin{array}{l}\text { Analyzing the disruption } \\
\text { resilience of bioenergy parks } \\
\text { using dynamic inoperability } \\
\text { input-output modeling }\end{array}$ & $\begin{array}{l}\text { Environment Systems } \\
\text { and Decisions }\end{array}$ & 2015 & 35 & 3 & 351 & 362 & $10.1007 / \mathrm{s} 10669-015-9562-5$ \\
\hline $\begin{array}{l}\text { Zhang Y., Zheng } \\
\text { H., Fath B.D. }\end{array}$ & $\begin{array}{l}\text { Ecological network analysis of } \\
\text { an industrial symbiosis system: } \\
\text { A case study of the Shandong } \\
\text { Lubei eco-industrial park }\end{array}$ & Ecological Modelling & 2015 & 306 & & 174 & 184 & 10.1016/j.ecolmodel.2014.05.005 \\
\hline $\begin{array}{l}\text { Beloborodko A., } \\
\text { Rosa M. }\end{array}$ & $\begin{array}{l}\text { The Use of Performance } \\
\text { Indicators for Analysis of } \\
\text { Resource Efficiency Measures }\end{array}$ & Energy Procedia & 2015 & 72 & & 337 & 344 & 10.1016/j.egypro.2015.06.049 \\
\hline $\begin{array}{l}\text { Yu C., Dijkema } \\
\text { G.P.J., de Jong } \\
\text { M. }\end{array}$ & $\begin{array}{c}\text { What Makes Eco- } \\
\text { Transformation of Industrial } \\
\text { Parks Take Off in China? }\end{array}$ & $\begin{array}{l}\text { Journal of Industrial } \\
\text { Ecology }\end{array}$ & 2015 & 19 & 3 & 441 & 456 & 10.1111/jiec. 12185 \\
\hline Lopes M.S.G. & $\begin{array}{c}\text { Engineering biological systems } \\
\text { toward a sustainable } \\
\text { bioeconomy }\end{array}$ & $\begin{array}{l}\text { Journal of Industrial } \\
\text { Microbiology and } \\
\text { Biotechnology }\end{array}$ & 2015 & 42 & 6 & 813 & 838 & $10.1007 / \mathrm{s} 10295-015-1606-9$ \\
\hline $\begin{array}{c}\text { Rončević B., Fric } \\
\text { U. }\end{array}$ & $\begin{array}{c}\text { Researching industrial } \\
\text { symbiosis: Challenges and } \\
\text { dilemmas }\end{array}$ & $\begin{array}{c}\text { Applied Modelling and } \\
\text { Computing in Social } \\
\text { Sciences }\end{array}$ & 2015 & & & 35 & 49 & $10.3726 / 978-3-653-05821-5$ \\
\hline $\begin{array}{l}\text { Päivärinne S., } \\
\text { Hjelm O., } \\
\text { Gustafsson S. }\end{array}$ & $\begin{array}{c}\text { Excess heat supply } \\
\text { collaborations within the } \\
\text { district heating sector: Drivers } \\
\text { and barriers }\end{array}$ & $\begin{array}{l}\text { Journal of Renewable } \\
\text { and Sustainable Energy }\end{array}$ & 2015 & 7 & 3 & & & $10.1063 / 1.4921759$ \\
\hline Li Y., Shi L. & $\begin{array}{c}\text { The Resilience of } \\
\text { Interdependent Industrial } \\
\text { Symbiosis Networks: A Case } \\
\text { of Yixing Economic and } \\
\text { Technological Development } \\
\text { Zone } \\
\end{array}$ & $\begin{array}{l}\text { Journal of Industrial } \\
\text { Ecology }\end{array}$ & 2015 & 19 & 2 & 264 & 273 & 10.1111/jiec. 12267 \\
\hline $\begin{array}{l}\text { Chandra-Putra } \\
\text { H., Chen J., } \\
\text { Andrews C.J. }\end{array}$ & $\begin{array}{l}\text { Eco-Evolutionary Pathways } \\
\text { Toward Industrial Cities }\end{array}$ & $\begin{array}{l}\text { Journal of Industrial } \\
\text { Ecology }\end{array}$ & 2015 & 19 & 2 & 274 & 284 & 10.1111/jiec. 12234 \\
\hline $\begin{array}{l}\text { Strazza C., } \\
\text { Magrassi F., } \\
\text { Gallo M., Del } \\
\text { Borghi A. } \\
\end{array}$ & $\begin{array}{l}\text { Life Cycle Assessment from } \\
\text { food to food: A case study of } \\
\text { circular economy from cruise } \\
\text { ships to aquaculture }\end{array}$ & $\begin{array}{l}\text { Sustainable Production } \\
\text { and Consumption }\end{array}$ & 2015 & 2 & & 40 & 51 & 10.1016/j.spc.2015.06.004 \\
\hline
\end{tabular}




\begin{tabular}{|c|c|c|c|c|c|c|c|c|}
\hline Author Name(s) & Title & Source Title & Year & Vol. & Issue & P. Start & P. End & Doi \\
\hline $\begin{array}{l}\text { Simboli A., } \\
\text { Taddeo R., } \\
\text { Morgante A. }\end{array}$ & $\begin{array}{c}\text { The potential of Industrial } \\
\text { Ecology in agri-food clusters } \\
\text { (AFCs): A case study based on } \\
\text { valorisation of auxiliary } \\
\text { materials }\end{array}$ & Ecological Economics & 2015 & 111 & & 65 & 75 & 10.1016/j.ecolecon.2015.01.005 \\
\hline $\begin{array}{l}\text { Bakshi B.R., Ziv } \\
\text { G., Lepech M.D. }\end{array}$ & $\begin{array}{l}\text { Techno-ecological synergy: A } \\
\text { framework for sustainable } \\
\text { engineering }\end{array}$ & $\begin{array}{l}\text { Environmental Science } \\
\text { and Technology }\end{array}$ & 2015 & 49 & 3 & 1752 & 1760 & $10.1021 / \mathrm{es} 5041442$ \\
\hline $\begin{array}{l}\text { Lam H.L., How } \\
\text { B.S., Hong B.H. }\end{array}$ & $\begin{array}{c}\text { Green supply chain toward } \\
\text { sustainable industry } \\
\text { development }\end{array}$ & $\begin{array}{c}\text { Assessing and } \\
\text { Measuring } \\
\text { Environmental Impact } \\
\text { and Sustainability }\end{array}$ & 2015 & & & 409 & 449 & 10.1016/B978-0-12-799968-5.00012-9 \\
\hline $\begin{array}{l}\text { Yu F., Han F., } \\
\text { Cui Z. }\end{array}$ & $\begin{array}{c}\text { Evolution of industrial } \\
\text { symbiosis in an eco-industrial } \\
\text { park in China }\end{array}$ & $\begin{array}{l}\text { Journal of Cleaner } \\
\text { Production }\end{array}$ & 2015 & 87 & $\mathrm{C}$ & 339 & 347 & 10.1016/j.jclepro.2014.10.058 \\
\hline $\begin{array}{c}\text { Chertow M., Park } \\
\text { J. }\end{array}$ & $\begin{array}{l}\text { Scholarship and practice in } \\
\text { industrial symbiosis: } 1989- \\
2014\end{array}$ & $\begin{array}{l}\text { Taking Stock of } \\
\text { Industrial Ecology }\end{array}$ & 2015 & & & 87 & 116 & 10.1007/978-3-319-20571-7_5 \\
\hline $\begin{array}{c}\text { Cecelja F., Raafat } \\
\text { T., Trokanas N., } \\
\text { Innes S., Smith } \\
\text { M., Yang A., } \\
\text { Zorgios Y., } \\
\text { Korkofygas A., } \\
\text { Kokossis A. }\end{array}$ & $\begin{array}{l}\text { E-Symbiosis: Technology- } \\
\text { enabled support for Industrial } \\
\text { Symbiosis targeting Small and } \\
\text { Medium Enterprises and } \\
\text { innovation }\end{array}$ & $\begin{array}{l}\text { Journal of Cleaner } \\
\text { Production }\end{array}$ & 2015 & 98 & & 336 & 352 & 10.1016/j.jclepro.2014.08.051 \\
\hline $\begin{array}{l}\text { Martin M., } \\
\text { Svensson N., } \\
\text { Eklund M. }\end{array}$ & $\begin{array}{l}\text { Who gets the benefits? An } \\
\text { approach for assessing the } \\
\text { environmental performance of } \\
\text { industrial symbiosis }\end{array}$ & $\begin{array}{l}\text { Journal of Cleaner } \\
\text { Production }\end{array}$ & 2015 & 98 & & 263 & 271 & 10.1016/j.jclepro.2013.06.024 \\
\hline $\begin{array}{l}\text { Hein A.M., } \\
\text { Jankovic M., } \\
\text { Farel R., Sam Lei } \\
\text { I., Yannou B. }\end{array}$ & $\begin{array}{l}\text { Modeling industrial symbiosis } \\
\text { using design structure matrices }\end{array}$ & $\begin{array}{c}\text { Modeling and } \\
\text { Managing Complex } \\
\text { Systems - Proceedings } \\
\text { of the 17th International } \\
\text { DSM Conference } \\
\end{array}$ & 2015 & & & 209 & 219 & \\
\hline $\begin{array}{c}\text { Paquin R.L., } \\
\text { Busch T., } \\
\text { Tilleman S.G. }\end{array}$ & $\begin{array}{c}\text { Creating economic and } \\
\text { environmental value through } \\
\text { industrial symbiosis }\end{array}$ & Long Range Planning & 2015 & 48 & 2 & 95 & 107 & 10.1016/j.lrp.2013.11.002 \\
\hline
\end{tabular}

APPLIED ECOLOGY AND ENVIRONMENTAL RESEARCH 17(1):1159-1221.

http://www.aloki.hu • ISSN 15891623 (Print) • ISSN 17850037 (Online)

DOI: http://dx.doi.org/10.15666/aeer/1701_11591221

(c) 2019, ALÖKI Kft., Budapest, Hungary 


\begin{tabular}{|c|c|c|c|c|c|c|c|c|}
\hline Author Name(s) & $\begin{array}{c}\text { Title } \\
\end{array}$ & Source Title & Year & Vol. & Issue & P. Start & P. End & Doi \\
\hline Martin M. & $\begin{array}{l}\text { Quantifying the environmental } \\
\text { performance of an industrial } \\
\text { symbiosis network of biofuel } \\
\text { producers }\end{array}$ & $\begin{array}{l}\text { Journal of Cleaner } \\
\text { Production }\end{array}$ & 2015 & 102 & & 202 & 212 & 10.1016/j.jclepro.2015.04.063 \\
\hline $\begin{array}{l}\text { Gaidajis G., } \\
\text { Kakanis I. }\end{array}$ & $\begin{array}{c}\text { Examination of industrial } \\
\text { symbiosis potential interactions } \\
\text { in an industrial area of NE } \\
\text { greece }\end{array}$ & $\begin{array}{c}\text { Journal of Engineering } \\
\text { Science and } \\
\text { Technology Review }\end{array}$ & 2015 & 8 & 3 & 130 & 135 & \\
\hline $\begin{array}{c}\text { Bertels S., Bowen } \\
\text { F. }\end{array}$ & $\begin{array}{l}\text { Taking Stock, Looking Ahead: } \\
\text { Editors' Introduction to the } \\
\text { Inaugural Organization \& } \\
\text { Environment Review Issue }\end{array}$ & $\begin{array}{l}\text { Organization and } \\
\text { Environment }\end{array}$ & 2015 & 28 & 1 & 3 & 7 & $10.1177 / 1086026615576798$ \\
\hline $\begin{array}{l}\text { Liu C., Côté R.P., } \\
\text { Zhang K. }\end{array}$ & $\begin{array}{c}\text { Implementing a three-level } \\
\text { approach in industrial } \\
\text { symbiosis }\end{array}$ & $\begin{array}{l}\text { Journal of Cleaner } \\
\text { Production }\end{array}$ & 2015 & 87 & 1 & 318 & 327 & 10.1016/j.jclepro.2014.09.067 \\
\hline Spekkink W. & $\begin{array}{l}\text { Building capacity for } \\
\text { sustainable regional industrial } \\
\text { systems: An event sequence } \\
\text { analysis of developments in the } \\
\text { Sloe Area and Canal Zone }\end{array}$ & $\begin{array}{l}\text { Journal of Cleaner } \\
\text { Production }\end{array}$ & 2015 & 98 & & 133 & 144 & 10.1016/j.jclepro.2014.08.028 \\
\hline $\begin{array}{l}\text { Bin S., Zhiquan } \\
\text { Y., Jonathan } \\
\text { L.S.C., Jiewei } \\
\text { D.K., Kurle D., } \\
\text { Cerdas F., } \\
\text { Herrmann C. }\end{array}$ & $\begin{array}{l}\text { A big data analytics approach } \\
\text { to develop industrial symbioses } \\
\text { in large cities }\end{array}$ & Procedia CIRP & 2015 & 29 & & 450 & 455 & 10.1016/j.procir.2015.01.066 \\
\hline $\begin{array}{l}\text { Aid G., Brandt } \\
\text { N., Lysenkova } \\
\text { M., Smedberg N. }\end{array}$ & $\begin{array}{l}\text { Looplocal - A heuristic } \\
\text { visualization tool to support the } \\
\text { strategic facilitation of } \\
\text { industrial symbiosis }\end{array}$ & $\begin{array}{l}\text { Journal of Cleaner } \\
\text { Production }\end{array}$ & 2015 & 98 & & 328 & 335 & 10.1016/j.jclepro.2014.08.012 \\
\hline $\begin{array}{c}\text { Trokanas N., } \\
\text { Cecelja F., Raafat } \\
\text { T. }\end{array}$ & $\begin{array}{l}\text { Semantic approach for pre- } \\
\text { assessment of environmental } \\
\text { indicators in Industrial } \\
\text { Symbiosis }\end{array}$ & $\begin{array}{l}\text { Journal of Cleaner } \\
\text { Production }\end{array}$ & 2015 & 96 & & 349 & 361 & 10.1016/j.jclepro.2013.12.046 \\
\hline $\begin{array}{c}\text { Rosa M., } \\
\text { Beloborodko A. }\end{array}$ & $\begin{array}{l}\text { A decision support method for } \\
\text { development of industrial } \\
\text { synergies: Case studies of } \\
\text { Latvian brewery and wood- } \\
\text { processing industries }\end{array}$ & $\begin{array}{l}\text { Journal of Cleaner } \\
\text { Production }\end{array}$ & 2015 & 105 & & 461 & 470 & 10.1016/j.jclepro.2014.09.061 \\
\hline
\end{tabular}

APPLIED ECOLOGY AND ENVIRONMENTAL RESEARCH 17(1):1159-1221.

http://www.aloki.hu • ISSN 15891623 (Print) • ISSN 17850037 (Online)

DOI: http://dx.doi.org/10.15666/aeer/1701_11591221

๑) 2019, ALÖKI Kft., Budapest, Hungary 


\begin{tabular}{|c|c|c|c|c|c|c|c|c|}
\hline Author Name(s) & Title & Source Title & Year & Vol. & Issue & P. Start & P. End & Doi \\
\hline Leigh M., Li X. & $\begin{array}{c}\text { Industrial ecology, industrial } \\
\text { symbiosis and supply chain } \\
\text { environmental sustainability: A } \\
\text { case study of a large UK } \\
\text { distributor } \\
\end{array}$ & $\begin{array}{l}\text { Journal of Cleaner } \\
\text { Production }\end{array}$ & 2015 & 106 & & 632 & 643 & 10.1016/j.jclepro.2014.09.022 \\
\hline $\begin{array}{l}\text { Trokanas N., } \\
\text { Bussemaker M., } \\
\text { Velliou E., Tokos } \\
\text { H., Cecelja F. } \\
\end{array}$ & $\begin{array}{c}\text { BiOnto: An Ontology for } \\
\text { Biomass and Biorefining } \\
\text { Technologies }\end{array}$ & $\begin{array}{c}\text { Computer Aided } \\
\text { Chemical Engineering }\end{array}$ & 2015 & 37 & & 959 & 964 & 10.1016/B978-0-444-63577-8.50005-X \\
\hline $\begin{array}{l}\text { Chudobiecki J., } \\
\text { Wanat L. }\end{array}$ & $\begin{array}{l}\text { Industrial symbiosis and green } \\
\text { business parks in the wood- } \\
\text { based sector in Poland }\end{array}$ & $\begin{array}{c}\text { Wood Processing and } \\
\text { Furniture } \\
\text { Manufacturing } \\
\text { Challenges on the } \\
\text { World Market and } \\
\text { Wood-Based Energy } \\
\text { Goes Global - } \\
\text { Proceedings of } \\
\text { Scientific Papers } \\
\end{array}$ & 2015 & & & 221 & 228 & \\
\hline $\begin{array}{l}\text { Albino V., } \\
\text { Fraccascia L., } \\
\text { Savino T. }\end{array}$ & $\begin{array}{c}\text { Industrial Symbiosis for a } \\
\text { Sustainable City: Technical, } \\
\text { Economical and Organizational } \\
\text { Issues }\end{array}$ & Procedia Engineering & 2015 & 118 & & 950 & 957 & 10.1016/j.proeng.2015.08.536 \\
\hline $\begin{array}{l}\text { Bailey M., Gadd } \\
\text { A. }\end{array}$ & $\begin{array}{l}\text { Quantifying the potential of } \\
\text { industrial symbiosis: The } \\
\text { LOCIMAP project, with } \\
\text { applications in the humber } \\
\text { region }\end{array}$ & $\begin{array}{l}\text { Taking Stock of } \\
\text { Industrial Ecology }\end{array}$ & 2015 & & & 343 & 357 & 10.1007/978-3-319-20571-7_19 \\
\hline $\begin{array}{l}\text { Yu B., Li X., Shi } \\
\text { L., Qian Y. }\end{array}$ & $\begin{array}{l}\text { Quantifying } \mathrm{CO} 2 \text { emission } \\
\text { reduction from industrial } \\
\text { symbiosis in integrated steel } \\
\text { mills in China }\end{array}$ & $\begin{array}{l}\text { Journal of Cleaner } \\
\text { Production }\end{array}$ & 2015 & 103 & & 801 & 810 & 10.1016/j.jclepro.2014.08.015 \\
\hline $\begin{array}{l}\text { Wu J., Li C., } \\
\text { Yang F. }\end{array}$ & $\begin{array}{c}\text { The disposition of chromite ore } \\
\text { processing residue (COPR) } \\
\text { incorporating industrial } \\
\text { symbiosis } \\
\end{array}$ & $\begin{array}{l}\text { Journal of Cleaner } \\
\text { Production }\end{array}$ & 2015 & 95 & & 156 & 162 & 10.1016/j.jclepro.2015.02.041 \\
\hline $\begin{array}{l}\text { Walls J.L., } \\
\text { Paquin R.L. }\end{array}$ & $\begin{array}{c}\text { Organizational Perspectives of } \\
\text { Industrial Symbiosis: A } \\
\text { Review and Synthesis }\end{array}$ & $\begin{array}{l}\text { Organization and } \\
\text { Environment }\end{array}$ & 2015 & 28 & 1 & 32 & 53 & $10.1177 / 1086026615575333$ \\
\hline
\end{tabular}




\begin{tabular}{|c|c|c|c|c|c|c|c|c|}
\hline Author Name(s) & Title & Source Title & Year & Vol. & Issue & P. Start & P. End & Doi \\
\hline $\begin{array}{l}\text { Golev A., Corder } \\
\text { G.D., Giurco D.P. }\end{array}$ & $\begin{array}{l}\text { Barriers to Industrial } \\
\text { Symbiosis: Insights from the } \\
\text { Use of a Maturity Grid }\end{array}$ & $\begin{array}{l}\text { Journal of Industrial } \\
\text { Ecology }\end{array}$ & 2015 & 19 & 1 & 141 & 153 & 10.1111/jiec.12159 \\
\hline $\begin{array}{l}\text { Puente M.C.R., } \\
\text { Arozamena E.R., } \\
\text { Evans S. }\end{array}$ & $\begin{array}{l}\text { Industrial symbiosis } \\
\text { opportunities for small and } \\
\text { medium sized enterprises: } \\
\text { Preliminary study in the } \\
\text { Besaya Region (Cantabria, } \\
\text { Northern Spain) } \\
\end{array}$ & $\begin{array}{l}\text { Journal of Cleaner } \\
\text { Production }\end{array}$ & 2015 & 87 & $\mathrm{C}$ & 357 & 374 & 10.1016/j.jclepro.2014.10.046 \\
\hline $\begin{array}{c}\text { Hein A.M., } \\
\text { Jankovic M., } \\
\text { Farel R., Yannou } \\
\text { B. } \\
\end{array}$ & $\begin{array}{l}\text { A conceptual framework for } \\
\text { eco-industrial parks }\end{array}$ & $\begin{array}{c}\text { Proceedings of the } \\
\text { ASME Design } \\
\text { Engineering Technical } \\
\text { Conference }\end{array}$ & 2015 & 4 & & & & 10.1115/DETC2015-46322 \\
\hline $\begin{array}{l}\text { Yu F., Han F., } \\
\text { Cui Z. }\end{array}$ & $\begin{array}{c}\text { Reducing carbon emissions } \\
\text { through industrial symbiosis: A } \\
\text { case study of a large enterprise } \\
\text { group in China }\end{array}$ & $\begin{array}{l}\text { Journal of Cleaner } \\
\text { Production }\end{array}$ & 2015 & 103 & & 811 & 818 & 10.1016/j.jclepro.2014.05.038 \\
\hline $\begin{array}{l}\text { Yu F., Han F., } \\
\text { Cui Z. }\end{array}$ & $\begin{array}{c}\text { Assessment of life cycle } \\
\text { environmental benefits of an } \\
\text { industrial symbiosis cluster in } \\
\text { China }\end{array}$ & $\begin{array}{l}\text { Environmental Science } \\
\text { and Pollution Research }\end{array}$ & 2015 & 22 & 7 & 5511 & 5518 & $10.1007 / \mathrm{s} 11356-014-3712-\mathrm{z}$ \\
\hline $\begin{array}{l}\text { Tsvetkova A., } \\
\text { Hellström M., } \\
\text { Gustafsson M., } \\
\text { Sjöblom J. }\end{array}$ & $\begin{array}{c}\text { Replication of industrial } \\
\text { ecosystems: The case of a } \\
\text { sustainable biogas-for-traffic } \\
\text { solution }\end{array}$ & $\begin{array}{l}\text { Journal of Cleaner } \\
\text { Production }\end{array}$ & 2015 & 98 & & 123 & 132 & 10.1016/j.jclepro.2014.08.089 \\
\hline $\begin{array}{l}\text { Päivärinne S., } \\
\text { Lindahl M. }\end{array}$ & $\begin{array}{c}\text { Exploratory study of } \\
\text { combining Integrated Product } \\
\text { and Services Offerings with } \\
\text { Industrial Symbiosis in order to } \\
\text { improve Excess Heat } \\
\text { utilization }\end{array}$ & Procedia CIRP & 2015 & 30 & & 167 & 172 & 10.1016/j.procir.2015.02.101 \\
\hline $\begin{array}{l}\text { Ammenberg J., } \\
\text { Baas L., Eklund } \\
\text { M., Feiz R., } \\
\text { Helgstrand A., } \\
\text { Marshall R. } \\
\end{array}$ & $\begin{array}{l}\text { Improving the } \mathrm{CO}<\mathrm{inf}>2</ \mathrm{inf}> \\
\text { performance of cement, part } \\
\text { III: The relevance of industrial } \\
\text { symbiosis and how to measure } \\
\text { its impact }\end{array}$ & $\begin{array}{l}\text { Journal of Cleaner } \\
\text { Production }\end{array}$ & 2015 & 98 & & 145 & 155 & 10.1016/j.jclepro.2014.01.086 \\
\hline
\end{tabular}




\begin{tabular}{|c|c|c|c|c|c|c|c|c|}
\hline Author Name(s) & Title & Source Title & Year & Vol. & Issue & P. Start & P. End & Doi \\
\hline Wen Z., Meng X. & $\begin{array}{l}\text { Quantitative assessment of } \\
\text { industrial symbiosis for the } \\
\text { promotion of circular economy: } \\
\text { A case study of the printed } \\
\text { circuit boards industry in } \\
\text { China's Suzhou New District }\end{array}$ & $\begin{array}{l}\text { Journal of Cleaner } \\
\text { Production }\end{array}$ & 2015 & 90 & & 211 & 219 & 10.1016/j.jclepro.2014.03.041 \\
\hline $\begin{array}{l}\text { Li H., Dong L., } \\
\text { Ren J. }\end{array}$ & $\begin{array}{c}\text { Industrial symbiosis as a } \\
\text { countermeasure for resource } \\
\text { dependent city: A case study of } \\
\text { Guiyang, China } \\
\end{array}$ & $\begin{array}{l}\text { Journal of Cleaner } \\
\text { Production }\end{array}$ & 2015 & 107 & & 252 & 266 & 10.1016/j.jclepro.2015.04.089 \\
\hline Kennedy C.A. & Industrial ecology and cities & $\begin{array}{c}\text { Taking Stock of } \\
\text { Industrial Ecology }\end{array}$ & 2015 & & & 69 & 86 & 10.1007/978-3-319-20571-7_4 \\
\hline Dong L., Fujita T. & $\begin{array}{l}\text { Promotion of low-carbon city } \\
\text { through industrial and urban } \\
\text { system innovation: Japanese } \\
\text { experience and China's } \\
\text { practice }\end{array}$ & $\begin{array}{l}\text { World Scientific } \\
\text { Reference on Asia and } \\
\text { the World Economy }\end{array}$ & 2015 & & & 257 & 279 & 10.1142/9789814578622_0033 \\
\hline $\begin{array}{l}\text { Puente M.C.R., } \\
\text { Arozamena E.R., } \\
\text { Evans S. }\end{array}$ & $\begin{array}{c}\text { Industrial symbiosis } \\
\text { opportunities for small and } \\
\text { medium sized enterprises: } \\
\text { Preliminary study in the } \\
\text { Besaya region (Cantabria, } \\
\text { Northern Spain) }\end{array}$ & $\begin{array}{l}\text { Journal of Cleaner } \\
\text { Production }\end{array}$ & 2015 & 87 & 1 & 357 & 374 & 10.1016/j.jclepro.2014.09.046 \\
\hline $\begin{array}{c}\text { Cutaia L., } \\
\text { Luciano A., } \\
\text { Barberio G., } \\
\text { Sbaffoni S., } \\
\text { Mancuso E., } \\
\text { Scagliarino C., La } \\
\text { Monica M. }\end{array}$ & $\begin{array}{l}\text { The experience of the first } \\
\text { industrial symbiosis platform in } \\
\text { Italy }\end{array}$ & $\begin{array}{c}\text { Environmental } \\
\text { Engineering and } \\
\text { Management Journal }\end{array}$ & 2015 & 14 & 7 & 1521 & 1533 & \\
\hline $\begin{array}{c}\text { Aviso K.B., Chiu } \\
\text { A.S.F., Yu } \\
\text { K.D.S., } \\
\text { Promentilla } \\
\text { M.A.B., Razon } \\
\text { L.F., Ubando } \\
\text { A.T., Sy C.L., } \\
\text { Tan R.R. } \\
\end{array}$ & $\begin{array}{c}\text { P-graph for optimising } \\
\text { industrial symbiotic networks }\end{array}$ & $\begin{array}{c}\text { Chemical Engineering } \\
\text { Transactions }\end{array}$ & 2015 & 45 & & 1345 & 1350 & 10.3303/CET1545225 \\
\hline
\end{tabular}




\begin{tabular}{|c|c|c|c|c|c|c|c|c|}
\hline Author Name(s) & $\begin{array}{c}\text { Title } \\
\end{array}$ & Source Title & Year & Vol. & Issue & P. Start & P. End & Doi \\
\hline $\begin{array}{l}\text { Vardanega R., } \\
\text { Prado J.M., } \\
\text { Meireles M.A.A. }\end{array}$ & $\begin{array}{l}\text { Adding value to agri-food } \\
\text { residues by means of } \\
\text { supercritical technology }\end{array}$ & $\begin{array}{c}\text { Journal of Supercritical } \\
\text { Fluids }\end{array}$ & 2015 & 96 & & 217 & 227 & 10.1016/j.supflu.2014.09.029 \\
\hline $\begin{array}{c}\text { Cerdas F., Kurle } \\
\text { D., Andrew S., } \\
\text { Thiede S., } \\
\text { Herrmann C., } \\
\text { Zhiquan Y., } \\
\text { Jonathan L.S.C., } \\
\text { Bin S., Kara S. } \\
\end{array}$ & $\begin{array}{l}\text { Defining circulation factories - } \\
\text { A pathway towards factories of } \\
\text { the future }\end{array}$ & Procedia CIRP & 2015 & 29 & & 627 & 632 & 10.1016/j.procir.2015.02.032 \\
\hline $\begin{array}{l}\text { Wang H., Xu X., } \\
\text { Zhu G. }\end{array}$ & $\begin{array}{l}\text { Landscape changes and a salt } \\
\text { production sustainable } \\
\text { approach in the state of salt pan } \\
\text { area decreasing on the coast of } \\
\text { Tianjin, China } \\
\end{array}$ & $\begin{array}{l}\text { Sustainability } \\
\text { (Switzerland) }\end{array}$ & 2015 & 7 & 8 & 10078 & 10097 & $10.3390 /$ su70810078 \\
\hline $\begin{array}{l}\text { Daddi T., } \\
\text { Tessitore S., } \\
\text { Testa F. }\end{array}$ & $\begin{array}{c}\text { Industrial ecology and eco- } \\
\text { industrial development: Case } \\
\text { studies from Italy }\end{array}$ & $\begin{array}{l}\text { Progress in Industrial } \\
\text { Ecology }\end{array}$ & 2015 & 9 & 3 & 217 & 233 & 10.1504/PIE.2015.073414 \\
\hline $\begin{array}{l}\text { Deutz P., Ioppolo } \\
\text { G. }\end{array}$ & $\begin{array}{l}\text { From theory to practice: } \\
\text { Enhancing the potential policy } \\
\text { impact of industrial ecology }\end{array}$ & $\begin{array}{l}\text { Sustainability } \\
\text { (Switzerland) }\end{array}$ & 2015 & 7 & 2 & 2259 & 2273 & $10.3390 / \mathrm{su} 7022259$ \\
\hline $\begin{array}{l}\text { [No author name } \\
\text { available] }\end{array}$ & $\begin{array}{c}\text { Modeling and Managing } \\
\text { Complex Systems - } \\
\text { Proceedings of the 17th } \\
\text { International DSM Conference }\end{array}$ & $\begin{array}{c}\text { Modeling and } \\
\text { Managing Complex } \\
\text { Systems - Proceedings } \\
\text { of the } 17 \text { th International } \\
\text { DSM Conference }\end{array}$ & 2015 & & & & & \\
\hline $\begin{array}{l}\text { Liu J., Nie X., } \\
\text { Zhou C., Shi Y., } \\
\text { Liu R. }\end{array}$ & $\begin{array}{l}\text { The design of agri-industrial } \\
\text { ecological park: A case study } \\
\text { of Zhengzhou national } \\
\text { economic-technological } \\
\text { development area }\end{array}$ & $\begin{array}{l}\text { Shengtai Xuebao/ Acta } \\
\text { Ecologica Sinica }\end{array}$ & 2015 & 35 & 14 & 4891 & 4896 & 10.5846/stxb201311242804 \\
\hline $\begin{array}{l}\text { Graedel T.E., } \\
\text { Lifset R.J. }\end{array}$ & $\begin{array}{c}\text { Industrial ecology's first } \\
\text { decade }\end{array}$ & $\begin{array}{c}\text { Taking Stock of } \\
\text { Industrial Ecology }\end{array}$ & 2015 & & & 3 & 20 & 10.1007/978-3-319-20571-7_1 \\
\hline $\begin{array}{l}\text { Adiansyah J.S., } \\
\text { Rosano M., Vink } \\
\text { S., Keir G. }\end{array}$ & $\begin{array}{l}\text { A framework for a sustainable } \\
\text { approach to mine tailings } \\
\text { management: Disposal } \\
\text { strategies }\end{array}$ & $\begin{array}{l}\text { Journal of Cleaner } \\
\text { Production }\end{array}$ & 2015 & 108 & & 1 & 13 & 10.1016/j.jclepro.2015.07.139 \\
\hline
\end{tabular}




\begin{tabular}{|c|c|c|c|c|c|c|c|c|}
\hline Author Name(s) & Title & Source Title & Year & Vol. & Issue & P. Start & P. End & Doi \\
\hline $\begin{array}{l}\text { Benjamin M.F., } \\
\text { Ubando A., } \\
\text { Razon L., Tan } \\
\text { R.R. }\end{array}$ & $\begin{array}{l}\text { Analyzing the disruption } \\
\text { resilience of microalgal multi- } \\
\text { functional Bioenergy systems } \\
\text { using dynamic inoperability } \\
\text { input-output modeling }\end{array}$ & $\begin{array}{c}\text { Chemical Engineering } \\
\text { Transactions }\end{array}$ & 2015 & 45 & & 1579 & 1584 & 10.3303/CET1545264 \\
\hline Johnson J.A. & $\begin{array}{l}\text { Dilemmas of 19th-century } \\
\text { liberalism among German } \\
\text { academic chemists: Shaping a } \\
\text { national science policy from } \\
\text { Hofmann to Fischer, 1865- } \\
1919 \\
\end{array}$ & Annals of Science & 2015 & 72 & 2 & 224 & 241 & $10.1080 / 00033790.2015 .1007525$ \\
\hline $\begin{array}{l}\text { Mannino I., } \\
\text { Ninka E., Turvani } \\
\text { M., Chertow M. } \\
\end{array}$ & $\begin{array}{c}\text { The decline of eco-industrial } \\
\text { development in Porto } \\
\text { Marghera, Italy }\end{array}$ & $\begin{array}{l}\text { Journal of Cleaner } \\
\text { Production }\end{array}$ & 2015 & 100 & & 286 & 296 & 10.1016/j.jclepro.2015.03.054 \\
\hline $\begin{array}{l}\text { Qu Y., Liu Y., } \\
\text { Nayak R.R., Li } \\
\text { M. }\end{array}$ & $\begin{array}{l}\text { Sustainable development of } \\
\text { eco-industrial parks in China: } \\
\text { Effects of managers' } \\
\text { environmental awareness on } \\
\text { the relationships between } \\
\text { practice and performance }\end{array}$ & $\begin{array}{l}\text { Journal of Cleaner } \\
\text { Production }\end{array}$ & 2015 & 87 & 1 & 328 & 338 & 10.1016/j.jclepro.2014.09.015 \\
\hline $\begin{array}{l}\text { Ubando A.T., } \\
\text { Culaba A.B., } \\
\text { Aviso K.B., Tan } \\
\text { R.R., Cuello J.L., } \\
\text { Ng D.K.S., El- } \\
\text { Halwagi M.M. } \\
\end{array}$ & $\begin{array}{l}\text { Fuzzy mathematical } \\
\text { programming approach in the } \\
\text { optimal design of an algal } \\
\text { bioenergy park }\end{array}$ & $\begin{array}{c}\text { Chemical Engineering } \\
\text { Transactions }\end{array}$ & 2015 & 45 & & 355 & 360 & 10.3303/CET1545060 \\
\hline $\begin{array}{l}\text { Olsson L., } \\
\text { Wetterlund E., } \\
\text { Söderström M. }\end{array}$ & $\begin{array}{l}\text { Assessing the climate impact of } \\
\text { district heating systems with } \\
\text { combined heat and power } \\
\text { production and industrial } \\
\text { excess heat }\end{array}$ & $\begin{array}{l}\text { Resources, } \\
\text { Conservation and } \\
\text { Recycling }\end{array}$ & 2015 & 96 & & 31 & 39 & 10.1016/j.resconrec.2015.01.006 \\
\hline $\begin{array}{c}\text { Li W., Cui Z., } \\
\text { Han F. }\end{array}$ & $\begin{array}{l}\text { Methods for assessing the } \\
\text { energy-saving efficiency of } \\
\text { industrial symbiosis in } \\
\text { industrial parks }\end{array}$ & $\begin{array}{c}\text { Environmental science } \\
\text { and pollution research } \\
\text { international }\end{array}$ & 2015 & 22 & 1 & 275 & 285 & $10.1007 / \mathrm{s} 11356-014-3327-4$ \\
\hline
\end{tabular}




\begin{tabular}{|c|c|c|c|c|c|c|c|c|}
\hline Author Name(s) & Title & Source Title & Year & Vol. & Issue & P. Start & P. End & Doi \\
\hline $\begin{array}{l}\text { Feiz R., } \\
\text { Ammenberg J., } \\
\text { Baas L., Eklund } \\
\text { M., Helgstrand } \\
\text { A., Marshall R. }\end{array}$ & $\begin{array}{c}\text { Improving the } \mathrm{CO}<\mathrm{inf}>2</ \mathrm{inf}> \\
\text { performance of cement, part II: } \\
\text { Framework for assessing } \\
\mathrm{CO}<\text { inf }>2</ \text { inf }>\text { improvement } \\
\text { measures in the cement } \\
\text { industry } \\
\end{array}$ & $\begin{array}{l}\text { Journal of Cleaner } \\
\text { Production }\end{array}$ & 2015 & 98 & & 282 & 291 & 10.1016/j.jclepro.2014.01.103 \\
\hline $\begin{array}{l}\text { Tan R.R., Ng } \\
\text { R.T.L., } \\
\text { Andiappan V., } \\
\text { Wan Y.K., Ng } \\
\text { D.K.S. } \\
\end{array}$ & $\begin{array}{l}\text { An optimization-based } \\
\text { cooperative game approach for } \\
\text { allocation of costs and benefits } \\
\text { in interplant process integration }\end{array}$ & $\begin{array}{c}\text { Chemical Engineering } \\
\text { Transactions }\end{array}$ & 2015 & 45 & & 403 & 408 & 10.3303/CET1545068 \\
\hline $\begin{array}{l}\text { Ferrão P., Lorena } \\
\text { A., Ribeiro P. }\end{array}$ & $\begin{array}{l}\text { Industrial ecology and } \\
\text { portugal's national waste plans }\end{array}$ & $\begin{array}{c}\text { Taking Stock of } \\
\text { Industrial Ecology }\end{array}$ & 2015 & & & 275 & 289 & 10.1007/978-3-319-20571-7_14 \\
\hline $\begin{array}{c}\text { Boix M., } \\
\text { Montastruc L., } \\
\text { Azzaro-Pantel C., } \\
\text { Domenech S. } \\
\end{array}$ & $\begin{array}{l}\text { Optimization methods applied } \\
\text { to the design of eco-industrial } \\
\text { parks: A literature review }\end{array}$ & $\begin{array}{l}\text { Journal of Cleaner } \\
\text { Production }\end{array}$ & 2015 & 87 & 1 & 303 & 317 & 10.1016/j.jclepro.2014.09.032 \\
\hline $\begin{array}{l}\text { Gregson N., } \\
\text { Crang M., Fuller } \\
\text { S., Holmes H. } \\
\end{array}$ & $\begin{array}{l}\text { Interrogating the circular } \\
\text { economy: the moral economy } \\
\text { of resource recovery in the EU }\end{array}$ & Economy and Society & 2015 & 44 & 2 & 218 & 243 & $10.1080 / 03085147.2015 .1013353$ \\
\hline $\begin{array}{c}\text { Noureldin } \\
\text { M.M.B., El- } \\
\text { Halwagi M.M. }\end{array}$ & $\begin{array}{c}\text { Synthesis of C-H-O Symbiosis } \\
\text { Networks }\end{array}$ & AIChE Journal & 2015 & 61 & 4 & 1242 & 1262 & 10.1002/aic.14714 \\
\hline $\begin{array}{c}\text { Duraccio V., } \\
\text { Gnoni M.G., Elia } \\
\text { V. }\end{array}$ & $\begin{array}{l}\text { Carbon capture and reuse in an } \\
\text { industrial district: A technical } \\
\text { and economic feasibility study }\end{array}$ & $\begin{array}{l}\text { Journal of } \mathrm{CO} 2 \\
\text { Utilization }\end{array}$ & 2015 & 10 & & 23 & 29 & 10.1016/j.jcou.2015.02.004 \\
\hline $\begin{array}{l}\text { Schieb P.-A., } \\
\text { Lescieux-Katir } \\
\text { H., Thénot M., } \\
\text { Clément- } \\
\text { Larosière B. } \\
\end{array}$ & $\begin{array}{l}\text { Biorefinery 2030: Future } \\
\text { prospects for the bioeconomy }\end{array}$ & $\begin{array}{l}\text { Biorefinery 2030: } \\
\text { Future Prospects for the } \\
\text { Bioeconomy }\end{array}$ & 2015 & & & 1 & 123 & $10.1007 / 978-3-662-47374-0$ \\
\hline $\begin{array}{l}\text { Ferrell J.C., } \\
\text { Shahbazi A. }\end{array}$ & $\begin{array}{l}\text { County government led EIP } \\
\text { development using municipal } \\
\text { biomass resources for clean } \\
\text { energy production, a case study } \\
\text { of the Catawba County North } \\
\text { Carolina ecocomplex }\end{array}$ & $\begin{array}{l}\text { Progress in Industrial } \\
\text { Ecology }\end{array}$ & 2015 & 9 & 1 & 69 & 81 & 10.1504/PIE.2015.069835 \\
\hline
\end{tabular}




\begin{tabular}{|c|c|c|c|c|c|c|c|c|}
\hline Author Name(s) & Title & Source Title & Year & Vol. & Issue & P. Start & P. End & Doi \\
\hline $\begin{array}{l}\text { Silva C.M.A., } \\
\text { Nielsen C.V., } \\
\text { Alves L.M.,, } \\
\text { Martins P.A.F. } \\
\end{array}$ & $\begin{array}{l}\text { Environmentally friendly } \\
\text { joining of tubes by their ends }\end{array}$ & $\begin{array}{l}\text { Journal of Cleaner } \\
\text { Production }\end{array}$ & 2015 & 87 & 1 & 777 & 786 & 10.1016/j.jclepro.2014.09.022 \\
\hline Rao P., Patil Y. & $\begin{array}{l}\text { Climate resilience in natural } \\
\text { ecosystems in india: } \\
\text { Technology adoption and the } \\
\text { use of local knowledge } \\
\text { processes and systems }\end{array}$ & $\begin{array}{l}\text { Handbook of Climate } \\
\text { Change Adaptation }\end{array}$ & 2015 & & & 2063 & 2077 & 10.1007/978-3-642-38670-1_95 \\
\hline $\begin{array}{l}\text { Marinos-Kouris } \\
\text { D., Mourtsiadis } \\
\text { A. }\end{array}$ & $\begin{array}{c}\text { Environment and recycling: } \\
\text { Some comments on the entropy } \\
\text { limits }\end{array}$ & $\begin{array}{c}\text { Fresenius } \\
\text { Environmental Bulletin }\end{array}$ & 2015 & 24 & 3B & 1158 & 1163 & \\
\hline Baas L., Hjelm O. & $\begin{array}{l}\text { Support your future today: } \\
\text { Enhancing sustainable } \\
\text { transitions by experimenting at } \\
\text { academic conferences }\end{array}$ & $\begin{array}{l}\text { Journal of Cleaner } \\
\text { Production }\end{array}$ & 2015 & 98 & & 1 & 7 & 10.1016/j.jclepro.2015.02.059 \\
\hline $\begin{array}{l}\text { Røyne F., Berlin } \\
\text { J., Ringström E. }\end{array}$ & $\begin{array}{l}\text { Life cycle perspective in } \\
\text { environmental strategy } \\
\text { development on the industry } \\
\text { cluster level: A case study of } \\
\text { five chemical companies }\end{array}$ & $\begin{array}{l}\text { Journal of Cleaner } \\
\text { Production }\end{array}$ & 2015 & 86 & & 125 & 131 & 10.1016/j.jclepro.2014.08.016 \\
\hline $\begin{array}{l}\text { Lenhart J., Van } \\
\text { Vliet B., Mol } \\
\text { A.P.J. }\end{array}$ & $\begin{array}{l}\text { New roles for local authorities } \\
\text { in a time of climate change: } \\
\text { The Rotterdam Energy } \\
\text { Approach and Planning as a } \\
\text { case of urban symbiosis }\end{array}$ & $\begin{array}{l}\text { Journal of Cleaner } \\
\text { Production }\end{array}$ & 2015 & 107 & & 593 & 601 & 10.1016/j.jclepro.2015.05.026 \\
\hline $\begin{array}{l}\text { Zhang Y., Zheng } \\
\text { H., Yang Z., Liu } \\
\text { G., Su M. }\end{array}$ & $\begin{array}{l}\text { Analysis of the industrial } \\
\text { metabolic processes for sulfur } \\
\text { in the Lubei (Shandong } \\
\text { Province, China) eco-industrial } \\
\text { park }\end{array}$ & $\begin{array}{l}\text { Journal of Cleaner } \\
\text { Production }\end{array}$ & 2015 & 96 & & 127 & 138 & 10.1016/j.jclepro.2014.01.096 \\
\hline Sahakian M. & $\begin{array}{c}\text { The social and solidarity } \\
\text { economy: Why is it relevant to } \\
\text { industrial ecology? }\end{array}$ & $\begin{array}{l}\text { Taking Stock of } \\
\text { Industrial Ecology }\end{array}$ & 2015 & & & 205 & 227 & 10.1007/978-3-319-20571-7_10 \\
\hline $\begin{array}{c}\text { Benjamin } \\
\text { M.F.D., Tan } \\
\text { R.R., Razon L.F. }\end{array}$ & $\begin{array}{l}\text { A methodology for criticality } \\
\text { analysis in integrated energy } \\
\text { systems }\end{array}$ & $\begin{array}{l}\text { Clean Technologies and } \\
\text { Environmental Policy }\end{array}$ & 2015 & 17 & 4 & 935 & 946 & $10.1007 / \mathrm{s} 10098-014-0846-0$ \\
\hline $\begin{array}{c}\text { Maillé M., } \\
\text { Frayret J.-M. }\end{array}$ & $\begin{array}{l}\text { Industrial Waste Reuse and By- } \\
\text { product Synergy Optimization }\end{array}$ & $\begin{array}{c}\text { Journal of Industrial } \\
\text { Ecology }\end{array}$ & 2016 & 20 & 6 & 1284 & 1294 & 10.1111/jiec. 12403 \\
\hline
\end{tabular}

APPLIED ECOLOGY AND ENVIRONMENTAL RESEARCH 17(1):1159-1221.

http://www.aloki.hu • ISSN 15891623 (Print) • ISSN 17850037 (Online)

DOI: http://dx.doi.org/10.15666/aeer/1701_11591221

( 2019 , ALÖKI Kft., Budapest, Hungary 


\begin{tabular}{|c|c|c|c|c|c|c|c|c|}
\hline Author Name(s) & Title & Source Title & Year & Vol. & Issue & P. Start & P. End & Doi \\
\hline $\begin{array}{l}\text { Papathanasoglou } \\
\text { A., Panagiotidou } \\
\text { M., Valta K., } \\
\text { Loizidou M. }\end{array}$ & $\begin{array}{l}\text { RESEARCH ARTICLE: } \\
\text { Institutional Barriers and } \\
\text { Opportunities for the } \\
\text { Implementation of Industrial } \\
\text { Symbiosis in Greece }\end{array}$ & Environmental Practice & 2016 & 18 & 4 & 253 & 259 & $10.1017 / S 1466046616000454$ \\
\hline $\begin{array}{l}\text { Wu J., Wang R., } \\
\text { Pu G., Qi H. }\end{array}$ & $\begin{array}{l}\text { Integrated assessment of } \\
\text { exergy, energy and carbon } \\
\text { dioxide emissions in an iron } \\
\text { and steel industrial network }\end{array}$ & Applied Energy & 2016 & 183 & & 430 & 444 & 10.1016/j.apenergy.2016.08.192 \\
\hline $\begin{array}{l}\text { Zhang B., Wang } \\
\text { Z., Lai K.-H. }\end{array}$ & $\begin{array}{l}\text { Does Industrial Waste Reuse } \\
\text { Bring Dual Benefits of } \\
\text { Economic Growth and Carbon } \\
\text { Emission Reduction?: } \\
\text { Evidence of Incorporating the } \\
\text { Indirect Effect of Economic } \\
\text { Growth in China }\end{array}$ & $\begin{array}{c}\text { Journal of Industrial } \\
\text { Ecology }\end{array}$ & 2016 & 20 & 6 & 1306 & 1319 & 10.1111/jiec. 12375 \\
\hline $\begin{array}{l}\text { Ubando A.T., } \\
\text { Culaba A.B., } \\
\text { Aviso K.B., Tan } \\
\text { R.R., Cuello J.L., } \\
\text { Ng D.K.S., El- } \\
\text { Halwagi M.M. }\end{array}$ & $\begin{array}{l}\text { Fuzzy mixed integer non-linear } \\
\text { programming model for the } \\
\text { design of an algae-based eco- } \\
\text { industrial park with prospective } \\
\text { selection of support tenants } \\
\text { under product price variability }\end{array}$ & $\begin{array}{l}\text { Journal of Cleaner } \\
\text { Production }\end{array}$ & 2016 & 136 & & 183 & 196 & 10.1016/j.jclepro.2016.04.143 \\
\hline $\begin{array}{l}\text { Peter Sahay S.S., } \\
\text { Dash S.N., Joga } \\
\text { Rao H. }\end{array}$ & $\begin{array}{l}\text { Economical benefit through } \\
\text { industrial symbiosis: Trash to } \\
\text { treasure: A case study in an } \\
\text { Indian industrial area } \\
\end{array}$ & $\begin{array}{l}\text { Research Journal of } \\
\text { Pharmaceutical, } \\
\text { Biological and } \\
\text { Chemical Sciences } \\
\end{array}$ & 2016 & 7 & 6 & 932 & 937 & \\
\hline $\begin{array}{l}\text { Wu J., Qi H., } \\
\text { Wang R. }\end{array}$ & $\begin{array}{c}\text { Insight into industrial } \\
\text { symbiosis and carbon } \\
\text { metabolism from the evolution } \\
\text { of iron and steel industrial } \\
\text { network }\end{array}$ & $\begin{array}{l}\text { Journal of Cleaner } \\
\text { Production }\end{array}$ & 2016 & 135 & & 251 & 262 & 10.1016/j.jclepro.2016.06.103 \\
\hline $\begin{array}{l}\text { Guo B., Geng Y., } \\
\text { Sterr T., Dong L., } \\
\text { Liu Y. }\end{array}$ & $\begin{array}{c}\text { Evaluation of promoting } \\
\text { industrial symbiosis in a } \\
\text { chemical industrial park: A } \\
\text { case of Midong } \\
\end{array}$ & $\begin{array}{l}\text { Journal of Cleaner } \\
\text { Production }\end{array}$ & 2016 & 135 & & 995 & 1008 & 10.1016/j.jclepro.2016.07.006 \\
\hline
\end{tabular}




\begin{tabular}{|c|c|c|c|c|c|c|c|c|}
\hline Author Name(s) & Title & Source Title & Year & Vol. & Issue & P. Start & P. End & Doi \\
\hline $\begin{array}{l}\text { Pan H., Zhang X., } \\
\text { Wang Y., Qi Y., } \\
\text { Wu J., Lin L., } \\
\text { Peng H., Qi H., } \\
\text { Yu X., Zhang Y. }\end{array}$ & $\begin{array}{l}\text { Emergy evaluation of an } \\
\text { industrial park in Sichuan } \\
\text { Province, China: A modified } \\
\text { emergy approach and its } \\
\text { application }\end{array}$ & $\begin{array}{l}\text { Journal of Cleaner } \\
\text { Production }\end{array}$ & 2016 & 135 & & 105 & 118 & 10.1016/j.jclepro.2016.06.102 \\
\hline $\begin{array}{l}\text { Álvarez R., Ruiz- } \\
\text { Puente C. }\end{array}$ & $\begin{array}{l}\text { Development of the Tool } \\
\text { SymbioSyS to Support the } \\
\text { Transition Towards a Circular } \\
\text { Economy Based on Industrial } \\
\text { Symbiosis Strategies }\end{array}$ & $\begin{array}{c}\text { Waste and Biomass } \\
\text { Valorization }\end{array}$ & 2016 & & & 1 & 10 & $10.1007 / \mathrm{s} 12649-016-9748-1$ \\
\hline $\begin{array}{l}\text { Taylor C.D., } \\
\text { Gully B., Sánchez } \\
\text { A.N., Rode E., } \\
\text { Agarwal A.S. }\end{array}$ & $\begin{array}{l}\text { Towards materials } \\
\text { sustainability through materials } \\
\text { stewardship }\end{array}$ & $\begin{array}{l}\text { Sustainability } \\
\text { (Switzerland) }\end{array}$ & 2016 & 8 & 10 & & & $10.3390 / \mathrm{su} 8101001$ \\
\hline $\begin{array}{c}\text { Atkins M.J., } \\
\text { Walmsley } \\
\text { M.R.W., } \\
\text { Walmsley T.G. }\end{array}$ & $\begin{array}{l}\text { Integration of new processes } \\
\text { and geothermal heat into a } \\
\text { wood processing cluster }\end{array}$ & $\begin{array}{c}\text { Clean Technologies and } \\
\text { Environmental Policy }\end{array}$ & 2016 & 18 & 7 & 2077 & 2085 & $10.1007 / \mathrm{s} 10098-016-1171-6$ \\
\hline Taddeo R. & $\begin{array}{l}\text { Local industrial systems } \\
\text { towards the eco-industrial } \\
\text { parks: The model of the } \\
\text { ecologically equipped } \\
\text { industrial areas }\end{array}$ & $\begin{array}{l}\text { Journal of Cleaner } \\
\text { Production }\end{array}$ & 2016 & 131 & & 189 & 197 & 10.1016/j.jclepro.2016.05.051 \\
\hline $\begin{array}{c}\text { Hodgson E., } \\
\text { Ruiz-Molina M.- } \\
\text { E., Marazza D., } \\
\text { Pogrebnyakova } \\
\text { E., Burns C., } \\
\text { Higson A., } \\
\text { Rehberger M., } \\
\text { Hiete M., Gyalai- } \\
\text { Korpos M., Lucia } \\
\text { L.D., Noël Y., } \\
\text { Woods J., } \\
\text { Gallagher J. } \\
\end{array}$ & $\begin{array}{l}\text { Horizon scanning the European } \\
\text { bio-based economy: a novel } \\
\text { approach to the identification } \\
\text { of barriers and key policy } \\
\text { interventions from stakeholders } \\
\text { in multiple sectors and regions }\end{array}$ & $\begin{array}{l}\text { Biofuels, Bioproducts } \\
\text { and Biorefining }\end{array}$ & 2016 & 10 & 5 & 508 & 522 & $10.1002 / \mathrm{bbb} .1665$ \\
\hline $\begin{array}{l}\text { Ren J., Liang H., } \\
\text { Dong L., Sun L., } \\
\text { Gao Z. }\end{array}$ & $\begin{array}{l}\text { Design for sustainability of } \\
\text { industrial symbiosis based on } \\
\text { emergy and multi-objective } \\
\text { particle swarm optimization }\end{array}$ & $\begin{array}{l}\text { Science of the Total } \\
\text { Environment }\end{array}$ & 2016 & 562 & & 789 & 801 & 10.1016/j.scitotenv.2016.04.092 \\
\hline
\end{tabular}




\begin{tabular}{|c|c|c|c|c|c|c|c|c|}
\hline Author Name(s) & Title & Source Title & Year & Vol. & Issue & P. Start & P. End & Doi \\
\hline $\begin{array}{l}\text { Secchi M., } \\
\text { Castellani V., } \\
\text { Collina E., } \\
\text { Mirabella N., } \\
\text { Sala S. } \\
\end{array}$ & $\begin{array}{l}\text { Assessing eco-innovations in } \\
\text { green chemistry: Life Cycle } \\
\text { Assessment (LCA) of a } \\
\text { cosmetic product with a bio- } \\
\text { based ingredient }\end{array}$ & $\begin{array}{l}\text { Journal of Cleaner } \\
\text { Production }\end{array}$ & 2016 & 129 & & 269 & 281 & 10.1016/j.jclepro.2016.04.073 \\
\hline Velenturf A.P.M. & $\begin{array}{c}\text { Promoting industrial } \\
\text { symbiosis: empirical } \\
\text { observations of low-carbon } \\
\text { innovations in the Humber } \\
\text { region, UK } \\
\end{array}$ & $\begin{array}{l}\text { Journal of Cleaner } \\
\text { Production }\end{array}$ & 2016 & 128 & & 116 & 130 & 10.1016/j.jclepro.2015.06.027 \\
\hline $\begin{array}{l}\text { Luciano A., } \\
\text { Barberio G., } \\
\text { Mancuso E., } \\
\text { Sbaffoni S., La } \\
\text { Monica M., } \\
\text { Scagliarino C., } \\
\text { Cutaia L. } \\
\end{array}$ & $\begin{array}{l}\text { Potential Improvement of the } \\
\text { Methodology for Industrial } \\
\text { Symbiosis Implementation at } \\
\text { Regional Scale }\end{array}$ & $\begin{array}{c}\text { Waste and Biomass } \\
\text { Valorization }\end{array}$ & 2016 & 7 & 4 & 1007 & 1015 & $10.1007 / \mathrm{s} 12649-016-9625-\mathrm{y}$ \\
\hline $\begin{array}{l}\text { Velenturf A.P.M., } \\
\text { Jensen P.D. }\end{array}$ & $\begin{array}{c}\text { Promoting Industrial } \\
\text { Symbiosis: Using the Concept } \\
\text { of Proximity to Explore Social } \\
\text { Network Development }\end{array}$ & $\begin{array}{c}\text { Journal of Industrial } \\
\text { Ecology }\end{array}$ & 2016 & 20 & 4 & 700 & 709 & 10.1111/jiec. 12315 \\
\hline $\begin{array}{c}\text { Renzulli P.A., } \\
\text { Notarnicola B., } \\
\text { Tassielli G., } \\
\text { Arcese G., Di } \\
\text { Capua R. } \\
\end{array}$ & $\begin{array}{l}\text { Life cycle assessment of steel } \\
\text { produced in an Italian } \\
\text { integrated steel mill }\end{array}$ & $\begin{array}{l}\text { Sustainability } \\
\text { (Switzerland) }\end{array}$ & 2016 & 8 & 8 & & & $10.3390 / \mathrm{su} 8080719$ \\
\hline $\begin{array}{l}\text { Liu Z.-Y., } \\
\text { Varbanov P.S., } \\
\text { Klemeš J.J., } \\
\text { Yong J.Y. }\end{array}$ & $\begin{array}{l}\text { Recent developments in } \\
\text { applied thermal engineering: } \\
\text { Process integration, heat } \\
\text { exchangers, enhanced heat } \\
\text { transfer, solar thermal energy, } \\
\text { combustion and high } \\
\text { temperature processes and } \\
\text { thermal process modelling }\end{array}$ & $\begin{array}{l}\text { Applied Thermal } \\
\text { Engineering }\end{array}$ & 2016 & 105 & & 755 & 762 & 10.1016/j.applthermaleng.2016.06.183 \\
\hline $\begin{array}{c}\text { Päivärinne S., } \\
\text { Lindahl M. }\end{array}$ & $\begin{array}{l}\text { Combining Integrated Product } \\
\text { and Service Offerings with } \\
\text { Industrial Symbiosis - a study } \\
\text { of opportunities and challenges }\end{array}$ & $\begin{array}{l}\text { Journal of Cleaner } \\
\text { Production }\end{array}$ & 2016 & 127 & & 240 & 248 & 10.1016/j.jclepro.2016.04.026 \\
\hline
\end{tabular}




\begin{tabular}{|c|c|c|c|c|c|c|c|c|}
\hline Author Name(s) & Title & Source Title & Year & Vol. & Issue & P. Start & P. End & Doi \\
\hline $\begin{array}{c}\text { Bacudio L.R., } \\
\text { Benjamin } \\
\text { M.F.D., Eusebio } \\
\text { R.C.P., Holaysan } \\
\text { S.A.K., } \\
\text { Promentilla } \\
\text { M.A.B., Yu } \\
\text { K.D.S., Aviso } \\
\text { K.B. } \\
\end{array}$ & $\begin{array}{c}\text { Analyzing barriers to } \\
\text { implementing industrial } \\
\text { symbiosis networks using } \\
\text { DEMATEL }\end{array}$ & $\begin{array}{l}\text { Sustainable Production } \\
\text { and Consumption }\end{array}$ & 2016 & 7 & & 57 & 65 & 10.1016/j.spc.2016.03.001 \\
\hline $\begin{array}{l}\text { Hu Y., Lin J., Cui } \\
\text { S., Khanna N.Z. }\end{array}$ & $\begin{array}{l}\text { Measuring Urban Carbon } \\
\text { Footprint from Carbon Flows } \\
\text { in the Global Supply Chain }\end{array}$ & $\begin{array}{l}\text { Environmental Science } \\
\text { and Technology }\end{array}$ & 2016 & 50 & 12 & 6154 & 6163 & 10.1021/acs.est.6b00985 \\
\hline $\begin{array}{c}\text { Zhang Y., Zheng } \\
\text { H., Shi H., Yu X., } \\
\text { Liu G., Su M., Li } \\
\text { Y., Chai Y. }\end{array}$ & $\begin{array}{l}\text { Network analysis of eight } \\
\text { industrial symbiosis systems }\end{array}$ & $\begin{array}{l}\text { Frontiers of Earth } \\
\text { Science }\end{array}$ & 2016 & 10 & 2 & 352 & 365 & $10.1007 / \mathrm{s} 11707-015-0520-9$ \\
\hline $\begin{array}{c}\text { Felicio M., } \\
\text { Amaral D., } \\
\text { Esposto K., } \\
\text { Gabarrell Durany } \\
\text { X. } \\
\end{array}$ & $\begin{array}{l}\text { Industrial symbiosis indicators } \\
\text { to manage eco-industrial parks } \\
\text { as dynamic systems }\end{array}$ & $\begin{array}{l}\text { Journal of Cleaner } \\
\text { Production }\end{array}$ & 2016 & 118 & & 54 & 64 & 10.1016/j.jclepro.2016.01.031 \\
\hline $\begin{array}{l}\text { Kikuchi Y., } \\
\text { Kanematsu Y., } \\
\text { Ugo M., Hamada } \\
\text { Y., Okubo T. } \\
\end{array}$ & $\begin{array}{l}\text { Industrial Symbiosis Centered } \\
\text { on a Regional Cogeneration } \\
\text { Power Plant Utilizing } \\
\text { Available Local Resources: A } \\
\text { Case Study of Tanegashima }\end{array}$ & $\begin{array}{l}\text { Journal of Industrial } \\
\text { Ecology }\end{array}$ & 2016 & 20 & 2 & 276 & 288 & 10.1111/jiec. 12347 \\
\hline $\begin{array}{c}\text { Kikuchi Y., } \\
\text { Kanematsu Y., } \\
\text { Sato R., Nakagaki } \\
\text { T. }\end{array}$ & $\begin{array}{l}\text { Distributed Cogeneration of } \\
\text { Power and Heat within an } \\
\text { Energy Management Strategy } \\
\text { for Mitigating Fossil Fuel } \\
\text { Consumption }\end{array}$ & $\begin{array}{l}\text { Journal of Industrial } \\
\text { Ecology }\end{array}$ & 2016 & 20 & 2 & 289 & 303 & 10.1111/jiec. 12374 \\
\hline $\begin{array}{l}\text { Ohnishi S., Fujii } \\
\text { M., Ohata M., } \\
\text { Rokuta I., Fujita } \\
\text { T. } \\
\end{array}$ & $\begin{array}{c}\text { Efficient energy recovery } \\
\text { through a combination of } \\
\text { waste-to-energy systems for a } \\
\text { low-carbon city }\end{array}$ & $\begin{array}{c}\text { Resources, } \\
\text { Conservation and } \\
\text { Recycling } \\
\end{array}$ & 2016 & & & & & 10.1016/j.resconrec.2016.11.018 \\
\hline
\end{tabular}




\begin{tabular}{|c|c|c|c|c|c|c|c|c|}
\hline Author Name(s) & $\begin{array}{c}\text { Title } \\
\end{array}$ & Source Title & Year & Vol. & Issue & P. Start & P. End & Doi \\
\hline $\begin{array}{l}\text { Liu Z., Adams } \\
\text { M., Cote R.P., } \\
\text { Geng Y., Li Y. }\end{array}$ & $\begin{array}{l}\text { Comparative study on the } \\
\text { pathways of industrial parks } \\
\text { towards sustainable } \\
\text { development between China } \\
\text { and Canada }\end{array}$ & $\begin{array}{l}\text { Resources, } \\
\text { Conservation and } \\
\text { Recycling }\end{array}$ & 2016 & & & & & 10.1016/j.resconrec.2016.06.012 \\
\hline $\begin{array}{l}\text { Kliopova I., } \\
\text { Baranauskaite- } \\
\text { Fedorova I., } \\
\text { Malinauskiene } \\
\text { M., Staniškis J.K. }\end{array}$ & $\begin{array}{l}\text { Possibilities of increasing } \\
\text { resource efficiency in nitrogen } \\
\text { fertilizer production }\end{array}$ & $\begin{array}{l}\text { Clean Technologies and } \\
\text { Environmental Policy }\end{array}$ & 2016 & 18 & 3 & 901 & 914 & $10.1007 / \mathrm{s} 10098-015-1068-9$ \\
\hline $\begin{array}{l}\text { Liu G., Hao Y., } \\
\text { Zhou Y., Yang } \\
\text { Z., Zhang Y., Su } \\
\text { M. } \\
\end{array}$ & $\begin{array}{c}\text { China's low-carbon industrial } \\
\text { transformation assessment } \\
\text { based on Logarithmic Mean } \\
\text { Divisia Index model } \\
\end{array}$ & $\begin{array}{l}\text { Resources, } \\
\text { Conservation and } \\
\text { Recycling }\end{array}$ & 2016 & 108 & & 156 & 170 & 10.1016/j.resconrec.2016.02.002 \\
\hline $\begin{array}{l}\text { Ghali M.R., } \\
\text { Frayret J.-M., } \\
\text { Robert J.-M. }\end{array}$ & $\begin{array}{l}\text { Green social networking: } \\
\text { Concept and potential } \\
\text { applications to initiate } \\
\text { industrial synergies }\end{array}$ & $\begin{array}{l}\text { Journal of Cleaner } \\
\text { Production }\end{array}$ & 2016 & 115 & & 23 & 35 & 10.1016/j.jclepro.2015.12.028 \\
\hline $\begin{array}{l}\text { Sun L., Li H., } \\
\text { Dong L., Fang } \\
\text { K., Ren J., Geng } \\
\text { Y., Fujii M., } \\
\text { Zhang W., Zhang } \\
\text { N., Liu Z. }\end{array}$ & $\begin{array}{l}\text { Eco-benefits assessment on } \\
\text { urban industrial symbiosis } \\
\text { based on material flows } \\
\text { analysis and emergy evaluation } \\
\text { approach: A case of Liuzhou } \\
\text { city, China }\end{array}$ & $\begin{array}{l}\text { Resources, } \\
\text { Conservation and } \\
\text { Recycling }\end{array}$ & 2016 & & & & & 10.1016/j.resconrec.2016.06.007 \\
\hline $\begin{array}{l}\text { Zhe L., Yong G., } \\
\text { Hung-Suck P., } \\
\text { Huijuan D., Liang } \\
\text { D., Tsuyoshi F. }\end{array}$ & $\begin{array}{l}\text { An emergy-based hybrid } \\
\text { method for assessing industrial } \\
\text { symbiosis of an industrial park }\end{array}$ & $\begin{array}{l}\text { Journal of Cleaner } \\
\text { Production }\end{array}$ & 2016 & 114 & & 132 & 140 & 10.1016/j.jclepro.2015.04.132 \\
\hline $\begin{array}{l}\text { Shiraki H., } \\
\text { Ashina S., } \\
\text { Kameyama Y., } \\
\text { Hashimoto S., } \\
\text { Fujita T. }\end{array}$ & $\begin{array}{l}\text { Analysis of optimal locations } \\
\text { for power stations and their } \\
\text { impact on industrial symbiosis } \\
\text { planning under transition } \\
\text { toward low-carbon power } \\
\text { sector in Japan } \\
\end{array}$ & $\begin{array}{l}\text { Journal of Cleaner } \\
\text { Production }\end{array}$ & 2016 & 114 & & 81 & 94 & 10.1016/j.jclepro.2015.09.079 \\
\hline $\begin{array}{l}\text { Park J.M., Park } \\
\text { J.Y., Park H.-S. }\end{array}$ & $\begin{array}{l}\text { A review of the National Eco- } \\
\text { Industrial Park Development } \\
\text { Program in Korea: Progress } \\
\text { and achievements in the first } \\
\text { phase, 2005-2010 }\end{array}$ & $\begin{array}{l}\text { Journal of Cleaner } \\
\text { Production }\end{array}$ & 2016 & 114 & & 33 & 44 & 10.1016/j.jclepro.2015.08.115 \\
\hline
\end{tabular}




\begin{tabular}{|c|c|c|c|c|c|c|c|c|}
\hline Author Name(s) & Title & Source Title & Year & Vol. & Issue & P. Start & P. End & Doi \\
\hline $\begin{array}{l}\text { Horváth G.Á., } \\
\text { Harazin P. }\end{array}$ & $\begin{array}{l}\text { A framework for an industrial } \\
\text { ecological decision support } \\
\text { system to foster partnerships } \\
\text { between businesses and } \\
\text { governments for sustainable } \\
\text { development } \\
\end{array}$ & $\begin{array}{l}\text { Journal of Cleaner } \\
\text { Production }\end{array}$ & 2016 & 114 & & 214 & 223 & 10.1016/j.jclepro.2015.05.018 \\
\hline $\begin{array}{l}\text { Mat N., Cerceau } \\
\text { J., Shi L., Park } \\
\text { H.-S., Junqua G., } \\
\text { Lopez-Ferber M. }\end{array}$ & $\begin{array}{l}\text { Socio-ecological transitions } \\
\text { toward low-carbon port cities: } \\
\text { Trends, changes and adaptation } \\
\text { processes in Asia and Europe }\end{array}$ & $\begin{array}{l}\text { Journal of Cleaner } \\
\text { Production }\end{array}$ & 2016 & 114 & & 362 & 375 & 10.1016/j.jclepro.2015.04.058 \\
\hline $\begin{array}{l}\text { Sumabat A.K., } \\
\text { Lopez N.S., Yu } \\
\text { K.D., Hao H., Li } \\
\text { R., Geng Y., Chiu } \\
\text { A.S.F. } \\
\end{array}$ & $\begin{array}{l}\text { Decomposition analysis of } \\
\text { Philippine } \mathrm{CO} 2 \text { emissions from } \\
\text { fuel combustion and electricity } \\
\text { generation }\end{array}$ & Applied Energy & 2016 & 164 & & 795 & 804 & 10.1016/j.apenergy.2015.12.023 \\
\hline $\begin{array}{l}\text { Daddi T., Iraldo } \\
\text { F., Frey M., Gallo } \\
\text { P., Gianfrate V. }\end{array}$ & $\begin{array}{l}\text { Regional policies and eco- } \\
\text { industrial development: The } \\
\text { voluntary environmental } \\
\text { certification scheme of the eco- } \\
\text { industrial parks in Tuscany } \\
\text { (Italy) }\end{array}$ & $\begin{array}{l}\text { Journal of Cleaner } \\
\text { Production }\end{array}$ & 2016 & 114 & & 62 & 70 & 10.1016/j.jclepro.2015.04.060 \\
\hline Jensen P.D. & $\begin{array}{l}\text { The role of geospatial } \\
\text { industrial diversity in the } \\
\text { facilitation of regional } \\
\text { industrial symbiosis }\end{array}$ & $\begin{array}{l}\text { Resources, } \\
\text { Conservation and } \\
\text { Recycling } \\
\end{array}$ & 2016 & 107 & & 92 & 103 & 10.1016/j.resconrec.2015.11.018 \\
\hline $\begin{array}{c}\text { Manara P., } \\
\text { Zabaniotou A. }\end{array}$ & $\begin{array}{l}\text { Co-valorization of Crude } \\
\text { Glycerol Waste Streams with } \\
\text { Conventional and/or } \\
\text { Renewable Fuels for Power } \\
\text { Generation and Industrial } \\
\text { Symbiosis Perspectives }\end{array}$ & $\begin{array}{l}\text { Waste and Biomass } \\
\text { Valorization }\end{array}$ & 2016 & 7 & 1 & 135 & 150 & $10.1007 / \mathrm{s} 12649-015-9439-3$ \\
\hline $\begin{array}{l}\text { Tan R.R., } \\
\text { Andiappan V., } \\
\text { Wan Y.K., Ng } \\
\text { R.T.L., Ng } \\
\text { D.K.S. } \\
\end{array}$ & $\begin{array}{l}\text { An optimization-based } \\
\text { cooperative game approach for } \\
\text { systematic allocation of costs } \\
\text { and benefits in interplant } \\
\text { process integration }\end{array}$ & $\begin{array}{l}\text { Chemical Engineering } \\
\text { Research and Design }\end{array}$ & 2016 & 106 & & 43 & 58 & 10.1016/j.cherd.2015.11.009 \\
\hline
\end{tabular}

APPLIED ECOLOGY AND ENVIRONMENTAL RESEARCH 17(1):1159-1221.

http://www.aloki.hu • ISSN 15891623 (Print) • ISSN 17850037 (Online)

DOI: http://dx.doi.org/10.15666/aeer/1701_11591221

(c) 2019, ALÖKI Kft., Budapest, Hungary 


\begin{tabular}{|c|c|c|c|c|c|c|c|c|}
\hline Author Name(s) & Title & Source Title & Year & Vol. & Issue & P. Start & P. End & Doi \\
\hline $\begin{array}{l}\text { Budzianowski } \\
\text { W.M. }\end{array}$ & $\begin{array}{c}\text { A review of potential } \\
\text { innovations for production, } \\
\text { conditioning and utilization of } \\
\text { biogas with multiple-criteria } \\
\text { assessment }\end{array}$ & $\begin{array}{c}\text { Renewable and } \\
\text { Sustainable Energy } \\
\text { Reviews }\end{array}$ & 2016 & 54 & & 1148 & 1171 & 10.1016/j.rser.2015.10.054 \\
\hline $\begin{array}{l}\text { Fernandez-Mena } \\
\text { H., Nesme T., } \\
\text { Pellerin S. }\end{array}$ & $\begin{array}{c}\text { Towards an Agro-Industrial } \\
\text { Ecology: A review of nutrient } \\
\text { flow modelling and assessment } \\
\text { tools in agro-food systems at } \\
\text { the local scale }\end{array}$ & $\begin{array}{l}\text { Science of the Total } \\
\text { Environment }\end{array}$ & 2016 & 543 & & 467 & 479 & 10.1016/j.scitotenv.2015.11.032 \\
\hline $\begin{array}{l}\text { Tiu B.T.C., Cruz } \\
\text { D.E. }\end{array}$ & $\begin{array}{c}\text { An MILP model for optimizing } \\
\text { water exchanges in eco- } \\
\text { industrial parks considering } \\
\text { water quality } \\
\end{array}$ & $\begin{array}{l}\text { Resources, } \\
\text { Conservation and } \\
\text { Recycling }\end{array}$ & 2016 & & & & & 10.1016/j.resconrec.2016.06.005 \\
\hline $\begin{array}{c}\text { Iacobescu R.I., } \\
\text { Angelopoulos } \\
\text { G.N., Jones P.T., } \\
\text { Blanpain B., } \\
\text { Pontikes Y. } \\
\end{array}$ & $\begin{array}{l}\text { Ladle metallurgy stainless steel } \\
\text { slag as a raw material in } \\
\text { Ordinary Portland Cement } \\
\text { production: A possibility for } \\
\text { industrial symbiosis }\end{array}$ & $\begin{array}{l}\text { Journal of Cleaner } \\
\text { Production }\end{array}$ & 2016 & 112 & & 872 & 881 & 10.1016/j.jclepro.2015.06.006 \\
\hline $\begin{array}{l}\text { Leong Y.T., Lee } \\
\text { J.-Y., Chew } \\
\text { I.M.L. }\end{array}$ & $\begin{array}{l}\text { Incorporating Timesharing } \\
\text { Scheme in Ecoindustrial } \\
\text { Multiperiod Chilled and } \\
\text { Cooling Water Network Design }\end{array}$ & $\begin{array}{c}\text { Industrial and } \\
\text { Engineering Chemistry } \\
\text { Research }\end{array}$ & 2016 & 55 & 1 & 197 & 209 & 10.1021/acs.iecr.5b02722 \\
\hline $\begin{array}{l}\text { Zhao Q., Shi } \\
\text { X.Q., Shi L. }\end{array}$ & $\begin{array}{c}\text { A review of the industrial } \\
\text { symbiosis network }\end{array}$ & $\begin{array}{c}\text { Shengtai Xuebao/ Acta } \\
\text { Ecologica Sinica }\end{array}$ & 2016 & 36 & 22 & 7288 & 7301 & 10.5846/stxb201507301598 \\
\hline $\begin{array}{l}\text { Albino V., } \\
\text { Fraccascia L., } \\
\text { Giannoccaro I. }\end{array}$ & $\begin{array}{l}\text { Exploring the role of contracts } \\
\text { to support the emergence of } \\
\text { self-organized industrial } \\
\text { symbiosis networks: An agent- } \\
\text { based simulation study }\end{array}$ & $\begin{array}{l}\text { Journal of Cleaner } \\
\text { Production }\end{array}$ & 2016 & 112 & & 4353 & 4366 & 10.1016/j.jclepro.2015.06.070 \\
\hline $\begin{array}{l}\text { Mantese G.C., De } \\
\text { Piere B.A., } \\
\text { Amaral D.C. }\end{array}$ & $\begin{array}{l}\text { A procedure to validate } \\
\text { industrial symbiosis indicators } \\
\text { combining conceptual and } \\
\text { empirical validation methods }\end{array}$ & $\begin{array}{l}\text { Advances in } \\
\text { Transdisciplinary } \\
\text { Engineering }\end{array}$ & 2016 & 4 & & 166 & 175 & 10.3233/978-1-61499-703-0-166 \\
\hline Branson R. & $\begin{array}{l}\text { Re-constructing Kalundborg: } \\
\text { The reality of bilateral } \\
\text { symbiosis and other insights }\end{array}$ & $\begin{array}{l}\text { Journal of Cleaner } \\
\text { Production }\end{array}$ & 2016 & 112 & & 4344 & 4352 & 10.1016/j.jclepro.2015.07.069 \\
\hline
\end{tabular}




\begin{tabular}{|c|c|c|c|c|c|c|c|c|}
\hline Author Name(s) & Title & Source Title & Year & Vol. & Issue & P. Start & P. End & Doi \\
\hline $\begin{array}{l}\text { Stratigaki C., } \\
\text { Loucopoulos P., } \\
\text { Migiakis A., } \\
\text { Zorgios Y. }\end{array}$ & $\begin{array}{l}\text { Combining model-driven and } \\
\text { capability-driven } \\
\text { developments: A case study of } \\
\text { industrial symbiosis }\end{array}$ & $\begin{array}{l}\text { CEUR Workshop } \\
\text { Proceedings }\end{array}$ & 2016 & 1753 & & 12 & 22 & \\
\hline $\begin{array}{l}\text { Liu J.R., Yan } \\
\text { Y.T., Nie X.R., } \\
\text { Yan L. }\end{array}$ & $\begin{array}{c}\text { The application of life cycle } \\
\text { assessments to the evaluation } \\
\text { of the environmental benefits } \\
\text { of industrial symbioses: } \\
\text { Research progress and } \\
\text { challenges }\end{array}$ & $\begin{array}{l}\text { Shengtai Xuebao/ Acta } \\
\text { Ecologica Sinica }\end{array}$ & 2016 & 36 & 22 & 7202 & 7207 & $10.5846 /$ stxb201411032156 \\
\hline $\begin{array}{l}\text { Boons F., } \\
\text { Chertow M., Park } \\
\text { J., Spekkink W., } \\
\text { Shi H. }\end{array}$ & $\begin{array}{c}\text { Industrial Symbiosis Dynamics } \\
\text { and the Problem of } \\
\text { Equivalence: Proposal for a } \\
\text { Comparative Framework }\end{array}$ & $\begin{array}{c}\text { Journal of Industrial } \\
\text { Ecology }\end{array}$ & 2016 & & & & & 10.1111/jiec. 12468 \\
\hline $\begin{array}{c}\text { Chattopadhyay } \\
\text { S., Kumar N., } \\
\text { Fine C., Olivetti } \\
\text { E. }\end{array}$ & $\begin{array}{c}\text { Industrial symbiosis among } \\
\text { small and medium scale } \\
\text { enterprises: Case of } \\
\text { Muzaffarnagar, India }\end{array}$ & TMS Annual Meeting & 2016 & & & 173 & 177 & \\
\hline $\begin{array}{c}\text { Dumoulin F., } \\
\text { Wassenaar T., } \\
\text { Avadí A., Paillat } \\
\text { J.-M. }\end{array}$ & $\begin{array}{l}\text { A Framework for Accurately } \\
\text { Informing Facilitated Regional } \\
\text { Industrial Symbioses on } \\
\text { Environmental Consequences }\end{array}$ & $\begin{array}{c}\text { Journal of Industrial } \\
\text { Ecology }\end{array}$ & 2016 & & & & & 10.1111/jiec. 12495 \\
\hline $\begin{array}{c}\text { [No author name } \\
\text { available] }\end{array}$ & CEUR Workshop Proceedings & $\begin{array}{l}\text { CEUR Workshop } \\
\text { Proceedings }\end{array}$ & 2016 & 1753 & & & & \\
\hline $\begin{array}{c}\text { Verguts V., } \\
\text { Dessein J., } \\
\text { Dewulf A., } \\
\text { Lauwers L., } \\
\text { Werkman R., } \\
\text { Termeer } \\
\text { C.J.A.M. }\end{array}$ & $\begin{array}{c}\text { Industrial symbiosis as } \\
\text { sustainable development } \\
\text { strategy: Adding a change } \\
\text { perspective }\end{array}$ & $\begin{array}{c}\text { International Journal of } \\
\text { Sustainable } \\
\text { Development }\end{array}$ & 2016 & 19 & 1 & 15 & 35 & 10.1504/IJSD.2016.073650 \\
\hline $\begin{array}{l}\text { Lignos G., } \\
\text { Stancari S., Bikos } \\
\text { S., Kokossis A. }\end{array}$ & $\begin{array}{c}\text { Structural and economic } \\
\text { analysis of Industrial } \\
\text { Symbiosis networks: a hybrid } \\
\text { approach to assess investment } \\
\text { opportunities }\end{array}$ & $\begin{array}{c}\text { Computer Aided } \\
\text { Chemical Engineering }\end{array}$ & 2016 & 38 & & 1617 & 1622 & 10.1016/B978-0-444-63428-3.50274-5 \\
\hline $\begin{array}{l}\text { Holgado M., } \\
\text { Morgan D., } \\
\text { Evans S. }\end{array}$ & $\begin{array}{c}\text { Exploring the scope of } \\
\text { industrial symbiosis: } \\
\text { Implications for practitioners }\end{array}$ & $\begin{array}{l}\text { Smart Innovation, } \\
\text { Systems and } \\
\text { Technologies }\end{array}$ & 2016 & 52 & & 169 & 178 & 10.1007/978-3-319-32098-4_15 \\
\hline
\end{tabular}

APPLIED ECOLOGY AND ENVIRONMENTAL RESEARCH 17(1):1159-1221.

http://www.aloki.hu • ISSN 15891623 (Print) • ISSN 17850037 (Online)

DOI: http://dx.doi.org/10.15666/aeer/1701_11591221

๑) 2019, ALÖKI Kft., Budapest, Hungary 


\begin{tabular}{|c|c|c|c|c|c|c|c|c|}
\hline Author Name(s) & Title & Source Title & Year & Vol. & Issue & P. Start & P. End & Doi \\
\hline $\begin{array}{l}\text { Hein A.M., } \\
\text { Jankovic M., } \\
\text { Farel R., Yannou } \\
\text { B. }\end{array}$ & $\begin{array}{l}\text { A data- and knowledge-driven } \\
\text { methodology for generating } \\
\text { ecoindustrial park architectures }\end{array}$ & $\begin{array}{c}\text { Proceedings of the } \\
\text { ASME Design } \\
\text { Engineering Technical } \\
\text { Conference }\end{array}$ & 2016 & 4 & & & & 10.1115/DETC2016-59171.pdf \\
\hline $\begin{array}{l}\text { Henkel M., } \\
\text { Stratigaki C., } \\
\text { Stirna J., } \\
\text { Loucopoulos P., } \\
\text { Zorgios Y., } \\
\text { Migiakis A. } \\
\end{array}$ & $\begin{array}{l}\text { Extending capabilities with } \\
\text { context awareness }\end{array}$ & $\begin{array}{l}\text { Lecture Notes in } \\
\text { Business Information } \\
\text { Processing }\end{array}$ & 2016 & 249 & & 40 & 51 & 10.1007/978-3-319-39564-7_4 \\
\hline $\begin{array}{l}\text { Holgado M., } \\
\text { Benedetti M., } \\
\text { Evans S., Introna } \\
\text { V. } \\
\end{array}$ & $\begin{array}{c}\text { Contextualisation in industrial } \\
\text { energy symbiosis: Design } \\
\text { process for a knowledge } \\
\text { repository } \\
\end{array}$ & $\begin{array}{l}\text { Proceedings of the } \\
\text { Summer School } \\
\text { Francesco Turco }\end{array}$ & 2016 & & & 139 & 144 & \\
\hline $\begin{array}{l}\text { Afshari H., } \\
\text { Gourlia J.-P., } \\
\text { Farel R., Peng Q. }\end{array}$ & $\begin{array}{l}\text { Energy symbioses in eco- } \\
\text { industrial parks: Models and } \\
\text { perspectives }\end{array}$ & $\begin{array}{c}\text { Proceedings of the } \\
\text { ASME Design } \\
\text { Engineering Technical } \\
\text { Conference }\end{array}$ & 2016 & 4 & & & & 10.1115/DETC2016-59965.pdf \\
\hline $\begin{array}{c}\text { Stirna J., } \\
\text { Zdravkovic J., } \\
\text { Henkel M., } \\
\text { Loucopoulos P., } \\
\text { Stratigaki C. } \\
\end{array}$ & $\begin{array}{c}\text { Modeling organizational } \\
\text { capabilities on a strategic level }\end{array}$ & $\begin{array}{l}\text { Lecture Notes in } \\
\text { Business Information } \\
\text { Processing }\end{array}$ & 2016 & 267 & & 257 & 271 & 10.1007/978-3-319-48393-1_18 \\
\hline $\begin{array}{c}\text { Siskos I., Van } \\
\text { Wassenhove L.N. }\end{array}$ & $\begin{array}{c}\text { Synergy Management Services } \\
\text { Companies: A New Business } \\
\text { Model for Industrial Park } \\
\text { Operators } \\
\end{array}$ & $\begin{array}{l}\text { Journal of Industrial } \\
\text { Ecology }\end{array}$ & 2016 & & & & & 10.1111/jiec. 12472 \\
\hline $\begin{array}{l}\text { Afshari H., Farel } \\
\text { R., Peng Q. }\end{array}$ & $\begin{array}{l}\text { Need for optimization under } \\
\text { uncertainty: Designing flow } \\
\text { exchanges in eco-industrial } \\
\text { parks }\end{array}$ & $\begin{array}{c}\text { Proceedings of the } \\
\text { ASME Design } \\
\text { Engineering Technical } \\
\text { Conference }\end{array}$ & 2016 & 4 & & & & 10.1115/DETC2016-59974.pdf \\
\hline $\begin{array}{l}\text { Mohammed F., } \\
\text { Biswas W.K., } \\
\text { Yao H., Tadé M. }\end{array}$ & $\begin{array}{c}\text { Identification of an } \\
\text { environmentally friendly } \\
\text { symbiotic process for the reuse } \\
\text { of industrial byproduct - An } \\
\text { LCA perspective }\end{array}$ & $\begin{array}{l}\text { Journal of Cleaner } \\
\text { Production }\end{array}$ & 2016 & 112 & & 3376 & 3387 & 10.1016/j.jclepro.2015.09.104 \\
\hline
\end{tabular}




\begin{tabular}{|c|c|c|c|c|c|c|c|c|}
\hline Author Name(s) & Title & Source Title & Year & Vol. & Issue & P. Start & P. End & Doi \\
\hline $\begin{array}{l}\text { Husgafvel R., } \\
\text { Nordlund H., } \\
\text { Heino J., Mäkelä } \\
\text { M., Watkins G., } \\
\text { Dahl O., Paavola } \\
\text { I.-L. } \\
\end{array}$ & $\begin{array}{c}\text { Use of Symbiosis Products } \\
\text { from Integrated Pulp and Paper } \\
\text { and Carbon Steel Mills: Legal } \\
\text { Status and Environmental } \\
\text { Burdens }\end{array}$ & $\begin{array}{l}\text { Journal of Industrial } \\
\text { Ecology }\end{array}$ & 2016 & 20 & 5 & 1187 & 1198 & 10.1111/jiec. 12348 \\
\hline $\begin{array}{l}\text { Qi Y., Zhu T., } \\
\text { Gao S., Wang } \\
\text { J.F., Ji Y.J., } \\
\text { Zhang M., Bu } \\
\text { X.X. }\end{array}$ & $\begin{array}{l}\text { Preliminary exploration of the } \\
\text { Chinese industrial park's } \\
\text { circularization reform using } \\
\text { key material flow analysis }\end{array}$ & $\begin{array}{l}\text { Shengtai Xuebao/ Acta } \\
\text { Ecologica Sinica }\end{array}$ & 2016 & 36 & 22 & 7335 & 7345 & 10.5846/stxb201508151708 \\
\hline $\begin{array}{l}\text { [No author name } \\
\text { available] }\end{array}$ & $\begin{array}{l}\text { 3rd International Conference } \\
\text { on Sustainable Design and } \\
\text { Manufacturing, SDM } 2016\end{array}$ & $\begin{array}{l}\text { Smart Innovation, } \\
\text { Systems and } \\
\text { Technologies } \\
\end{array}$ & 2016 & 52 & & 1 & 688 & \\
\hline $\begin{array}{l}\text { Kanematsu Y., } \\
\text { Oosawa K., } \\
\text { Kikuchi Y. }\end{array}$ & Agriculture & $\begin{array}{c}\text { Energy Technology } \\
\text { Roadmaps of Japan: } \\
\text { Future Energy Systems } \\
\text { Based on Feasible } \\
\text { Technologies Beyond } \\
2030 \\
\end{array}$ & 2016 & & & 405 & 414 & 10.1007/978-4-431-55951-1_27 \\
\hline $\begin{array}{l}\text { De Souza V., } \\
\text { Borsato M., } \\
\text { Bloemhof J. }\end{array}$ & $\begin{array}{c}\text { Designing eco-effective reverse } \\
\text { logistics networks }\end{array}$ & $\begin{array}{c}\text { Advances in } \\
\text { Transdisciplinary } \\
\text { Engineering }\end{array}$ & 2016 & 4 & & 851 & 860 & $10.3233 / 978-1-61499-703-0-851$ \\
\hline $\begin{array}{l}\text { Leong Y.T., Tan } \\
\text { R.R., Aviso K.B., } \\
\text { Chew I.M.L. }\end{array}$ & $\begin{array}{c}\text { Fuzzy analytic hierarchy } \\
\text { process and targeting for inter- } \\
\text { plant chilled and cooling water } \\
\text { network synthesis } \\
\end{array}$ & $\begin{array}{l}\text { Journal of Cleaner } \\
\text { Production }\end{array}$ & 2016 & 110 & & 40 & 53 & 10.1016/j.jclepro.2015.02.036 \\
\hline $\begin{array}{l}\text { Shi L., Chen } \\
\text { W.Q. }\end{array}$ & $\begin{array}{l}\text { Industrial ecology in China: } \\
\text { Retrospect and prospect }\end{array}$ & $\begin{array}{l}\text { Shengtai Xuebao/ Acta } \\
\text { Ecologica Sinica }\end{array}$ & 2016 & 36 & 22 & 7158 & 7167 & $10.5846 /$ stxb201611232387 \\
\hline $\begin{array}{l}\text { Kikuchi Y., } \\
\text { Kanematsu Y., } \\
\text { Okubo T. }\end{array}$ & $\begin{array}{l}\text { A computer-aided scenario } \\
\text { analysis of national and } \\
\text { regional energy systems based } \\
\text { on feasible technology options }\end{array}$ & $\begin{array}{c}\text { Computer Aided } \\
\text { Chemical Engineering }\end{array}$ & 2016 & 38 & & 1959 & 1964 & 10.1016/B978-0-444-63428-3.50331-3 \\
\hline $\begin{array}{l}\text { Yong J.Y., } \\
\text { Klemeš J.J., } \\
\text { Varbanov P.S., } \\
\text { Huisingh D. }\end{array}$ & $\begin{array}{l}\text { Cleaner energy for cleaner } \\
\text { production: Modelling, } \\
\text { simulation, optimisation and } \\
\text { waste management }\end{array}$ & $\begin{array}{l}\text { Journal of Cleaner } \\
\text { Production }\end{array}$ & 2016 & 111 & & 1 & 16 & 10.1016/j.jclepro.2015.10.062 \\
\hline $\begin{array}{l}\text { Petek J., Glavič } \\
\text { P., Kostevšek A. }\end{array}$ & $\begin{array}{c}\text { Total Site Resource Efficiency } \\
\text { System }\end{array}$ & $\begin{array}{c}\text { Computer Aided } \\
\text { Chemical Engineering } \\
\end{array}$ & 2016 & 38 & & 2235 & 2240 & 10.1016/B978-0-444-63428-3.50377-5 \\
\hline
\end{tabular}

APPLIED ECOLOGY AND ENVIRONMENTAL RESEARCH 17(1):1159-1221.

http://www.aloki.hu • ISSN 15891623 (Print) • ISSN 17850037 (Online)

DOI: http://dx.doi.org/10.15666/aeer/1701_11591221

(c) 2019, ALÖKI Kft., Budapest, Hungary 


\begin{tabular}{|c|c|c|c|c|c|c|c|c|}
\hline Author Name(s) & Title & Source Title & Year & Vol. & Issue & P. Start & P. End & Doi \\
\hline $\begin{array}{l}\text { Yazan D.M., } \\
\text { Romano V.A., } \\
\text { Albino V. }\end{array}$ & $\begin{array}{l}\text { The design of industrial } \\
\text { symbiosis: An input-output } \\
\text { approach }\end{array}$ & $\begin{array}{l}\text { Journal of Cleaner } \\
\text { Production }\end{array}$ & 2016 & & & & & 10.1016/j.jclepro.2016.03.160 \\
\hline $\begin{array}{l}\text { Kuznetsova E., } \\
\text { Zio E., Farel R. }\end{array}$ & $\begin{array}{l}\text { A methodological framework } \\
\text { for Eco-Industrial Park design } \\
\text { and optimization }\end{array}$ & $\begin{array}{l}\text { Journal of Cleaner } \\
\text { Production }\end{array}$ & 2016 & & & & & 10.1016/j.jclepro.2016.03.025 \\
\hline $\begin{array}{l}\text { Notarnicola B., } \\
\text { Tassielli G., } \\
\text { Renzulli P.A. }\end{array}$ & $\begin{array}{l}\text { Industrial symbiosis in the } \\
\text { Taranto industrial district: } \\
\text { Current level, constraints and } \\
\text { potential new synergies }\end{array}$ & $\begin{array}{l}\text { Journal of Cleaner } \\
\text { Production }\end{array}$ & 2016 & & & & & 10.1016/j.jclepro.2016.02.056 \\
\hline $\begin{array}{l}\text { Szabó S., Bódis } \\
\text { K., Kougias I., } \\
\text { Moner-Girona } \\
\text { M., Jäger-Waldau } \\
\text { A., Barton G., } \\
\text { Szabó L. } \\
\end{array}$ & $\begin{array}{c}\text { A methodology for maximizing } \\
\text { the benefits of solar landfills on } \\
\text { closed sites }\end{array}$ & $\begin{array}{c}\text { Renewable and } \\
\text { Sustainable Energy } \\
\text { Reviews }\end{array}$ & 2017 & 76 & & 1291 & 1300 & 10.1016/j.rser.2017.03.117 \\
\hline $\begin{array}{c}\text { Fraccascia L., } \\
\text { Giannoccaro I., } \\
\text { Albino V. }\end{array}$ & $\begin{array}{c}\text { Rethinking Resilience in } \\
\text { Industrial Symbiosis: } \\
\text { Conceptualization and } \\
\text { Measurements } \\
\end{array}$ & Ecological Economics & 2017 & 137 & & 148 & 162 & 10.1016/j.ecolecon.2017.02.026 \\
\hline $\begin{array}{c}\text { Wu J., Guo Y., Li } \\
\text { C., Qi H. }\end{array}$ & $\begin{array}{c}\text { The redundancy of an } \\
\text { industrial symbiosis network: } \\
\text { A case study of a hazardous } \\
\text { waste symbiosis network }\end{array}$ & $\begin{array}{l}\text { Journal of Cleaner } \\
\text { Production }\end{array}$ & 2017 & 149 & & 49 & 59 & 10.1016/j.jclepro.2017.02.038 \\
\hline $\begin{array}{c}\text { Wang D., Li J., } \\
\text { Wang Y., Wan } \\
\text { K., Song X., Liu } \\
\text { Y. } \\
\end{array}$ & $\begin{array}{l}\text { Comparing the vulnerability of } \\
\text { different coal industrial } \\
\text { symbiosis networks under } \\
\text { economic fluctuations }\end{array}$ & $\begin{array}{l}\text { Journal of Cleaner } \\
\text { Production }\end{array}$ & 2017 & 149 & & 636 & 652 & 10.1016/j.jclepro.2017.02.137 \\
\hline $\begin{array}{l}\text { Desrochers P., } \\
\text { Szurmak J. }\end{array}$ & $\begin{array}{l}\text { Long distance trade, locational } \\
\text { dynamics and by-product } \\
\text { development: Insights from the } \\
\text { history of the American } \\
\text { cottonseed industry }\end{array}$ & $\begin{array}{l}\text { Sustainability } \\
\text { (Switzerland) }\end{array}$ & 2017 & 9 & 4 & & & $10.3390 / \mathrm{su} 9040579$ \\
\hline $\begin{array}{c}\text { Sun L., Spekkink } \\
\text { W., Cuppen E., } \\
\text { Korevaar G. }\end{array}$ & $\begin{array}{l}\text { Coordination of industrial } \\
\text { symbiosis through anchoring }\end{array}$ & $\begin{array}{l}\text { Sustainability } \\
\text { (Switzerland) }\end{array}$ & 2017 & 9 & 4 & & & $10.3390 /$ su 9040549 \\
\hline
\end{tabular}




\begin{tabular}{|c|c|c|c|c|c|c|c|c|}
\hline Author Name(s) & Title & Source Title & Year & Vol. & Issue & P. Start & P. End & Doi \\
\hline $\begin{array}{l}\text { Hein A.M., } \\
\text { Jankovic M., } \\
\text { Feng W., Farel } \\
\text { R., Yune J.H., } \\
\text { Yannou B. }\end{array}$ & $\begin{array}{l}\text { Stakeholder power in industrial } \\
\text { symbioses: A stakeholder value } \\
\text { network approach }\end{array}$ & $\begin{array}{l}\text { Journal of Cleaner } \\
\text { Production }\end{array}$ & 2017 & 148 & & 923 & 933 & 10.1016/j.jclepro.2017.01.136 \\
\hline $\begin{array}{l}\text { Sun L., Li H., } \\
\text { Dong L., Fang } \\
\text { K., Ren J., Geng } \\
\text { Y., Fujii M., } \\
\text { Zhang W., Zhang } \\
\text { N., Liu Z. }\end{array}$ & $\begin{array}{l}\text { Eco-benefits assessment on } \\
\text { urban industrial symbiosis } \\
\text { based on material flows } \\
\text { analysis and emergy evaluation } \\
\text { approach: A case of Liuzhou } \\
\text { city, China }\end{array}$ & $\begin{array}{l}\text { Resources, } \\
\text { Conservation and } \\
\text { Recycling }\end{array}$ & 2017 & 119 & & 78 & 88 & 10.1016/j.resconrec.2016.06.007 \\
\hline $\begin{array}{l}\text { Tiu B.T.C., Cruz } \\
\text { D.E. }\end{array}$ & $\begin{array}{c}\text { An MILP model for optimizing } \\
\text { water exchanges in eco- } \\
\text { industrial parks considering } \\
\text { water quality }\end{array}$ & $\begin{array}{l}\text { Resources, } \\
\text { Conservation and } \\
\text { Recycling }\end{array}$ & 2017 & 119 & & 89 & 96 & 10.1016/j.resconrec.2016.06.005 \\
\hline $\begin{array}{l}\text { Fraccascia L., } \\
\text { Giannoccaro I., } \\
\text { Albino V. }\end{array}$ & $\begin{array}{l}\text { Efficacy of landfill tax and } \\
\text { subsidy policies for the } \\
\text { emergence of industrial } \\
\text { symbiosis networks: An agent- } \\
\text { based simulation study }\end{array}$ & $\begin{array}{l}\text { Sustainability } \\
\text { (Switzerland) }\end{array}$ & 2017 & 9 & 4 & & & $10.3390 / \mathrm{su} 9040521$ \\
\hline $\begin{array}{l}\text { Gabriel M., } \\
\text { Schöggl J.-P., } \\
\text { Posch A. }\end{array}$ & $\begin{array}{c}\text { Early front-end innovation } \\
\text { decisions for self-organized } \\
\text { industrial symbiosis dynamics- } \\
\text { A case study on lignin } \\
\text { utilization }\end{array}$ & $\begin{array}{l}\text { Sustainability } \\
\text { (Switzerland) }\end{array}$ & 2017 & 9 & 4 & & & $10.3390 / \mathrm{su} 9040515$ \\
\hline $\begin{array}{l}\text { Saraceni A.V., } \\
\text { Resende L.M., de } \\
\text { Andrade Júnior } \\
\text { P.P., Pontes J. }\end{array}$ & $\begin{array}{l}\text { Pilot testing model to uncover } \\
\text { industrial symbiosis in } \\
\text { Brazilian industrial clusters }\end{array}$ & $\begin{array}{l}\text { Environmental Science } \\
\text { and Pollution Research }\end{array}$ & 2017 & 24 & 12 & 11618 & 11629 & 10.1007/s11356-017-8794-y \\
\hline $\begin{array}{l}\text { Daddi T., Nucci } \\
\text { B., Iraldo F. }\end{array}$ & $\begin{array}{c}\text { Using Life Cycle Assessment } \\
\text { (LCA) to measure the } \\
\text { environmental benefits of } \\
\text { industrial symbiosis in an } \\
\text { industrial cluster of SMEs } \\
\end{array}$ & $\begin{array}{l}\text { Journal of Cleaner } \\
\text { Production }\end{array}$ & 2017 & 147 & & 157 & 164 & 10.1016/j.jclepro.2017.01.090 \\
\hline Mauthoor S. & $\begin{array}{c}\text { Uncovering industrial } \\
\text { symbiosis potentials in a small } \\
\text { island developing state: The } \\
\text { case study of Mauritius }\end{array}$ & $\begin{array}{l}\text { Journal of Cleaner } \\
\text { Production }\end{array}$ & 2017 & 147 & & 506 & 513 & 10.1016/j.jclepro.2017.01.138 \\
\hline
\end{tabular}




\begin{tabular}{|c|c|c|c|c|c|c|c|c|}
\hline Author Name(s) & Title & Source Title & Year & Vol. & Issue & P. Start & P. End & Doi \\
\hline $\begin{array}{l}\text { Liu Z., Adams } \\
\text { M., Cote R.P., } \\
\text { Geng Y., Chen } \\
\text { Q., Liu W., Sun } \\
\text { L., Yu X. }\end{array}$ & $\begin{array}{c}\text { Comprehensive development } \\
\text { of industrial symbiosis for the } \\
\text { response of greenhouse gases } \\
\text { emission mitigation: } \\
\text { Challenges and opportunities in } \\
\text { China } \\
\end{array}$ & Energy Policy & 2017 & 102 & & 88 & 95 & 10.1016/j.enpol.2016.12.013 \\
\hline $\begin{array}{l}\text { Serdar M., } \\
\text { Biljecki I., } \\
\text { Bjegović D. }\end{array}$ & $\begin{array}{c}\text { High-performance concrete } \\
\text { incorporating locally available } \\
\text { industrial by-products }\end{array}$ & $\begin{array}{l}\text { Journal of Materials in } \\
\text { Civil Engineering }\end{array}$ & 2017 & 29 & 3 & & & 10.1061/(ASCE)MT.1943-5533.0001773 \\
\hline $\begin{array}{c}\text { Yedla S., Park } \\
\text { H.-S. }\end{array}$ & $\begin{array}{l}\text { Eco-industrial networking for } \\
\text { sustainable development: } \\
\text { review of issues and } \\
\text { development strategies }\end{array}$ & $\begin{array}{c}\text { Clean Technologies and } \\
\text { Environmental Policy }\end{array}$ & 2017 & 19 & 2 & 391 & 402 & 10.1007/s10098-016-1224-х \\
\hline $\begin{array}{l}\text { Ubando A.T., } \\
\text { Aguilar K.D.T. }\end{array}$ & $\begin{array}{l}\text { Fuzzy quadratic programming } \\
\text { model for the optimal design of } \\
\text { an algal bioenergy park under } \\
\text { optimal price markdown } \\
\text { percentage }\end{array}$ & $\begin{array}{l}\text { IEEE Region } 10 \text { Annual } \\
\text { International } \\
\text { Conference, } \\
\text { Proceedings/TENCON }\end{array}$ & 2017 & & & 936 & 941 & 10.1109/TENCON.2016.7848142 \\
\hline $\begin{array}{l}\text { Leong Y.T., Lee } \\
\text { J.-Y., Tan R.R., } \\
\text { Foo J.J., Chew } \\
\text { I.M.L. }\end{array}$ & $\begin{array}{l}\text { Multi-objective optimization } \\
\text { for resource network synthesis } \\
\text { in eco-industrial parks using an } \\
\text { integrated analytic hierarchy } \\
\text { process }\end{array}$ & $\begin{array}{l}\text { Journal of Cleaner } \\
\text { Production }\end{array}$ & 2017 & 143 & & 1268 & 1283 & 10.1016/j.jclepro.2016.11.147 \\
\hline $\begin{array}{l}\text { Ceglia D., Abreu } \\
\text { M.C.S.D., Da } \\
\text { Silva Filho J.C.L. }\end{array}$ & $\begin{array}{l}\text { Critical elements for eco- } \\
\text { retrofitting a conventional } \\
\text { industrial park: Social barriers } \\
\text { to be overcome }\end{array}$ & $\begin{array}{c}\text { Journal of } \\
\text { Environmental } \\
\text { Management }\end{array}$ & 2017 & 187 & & 375 & 383 & 10.1016/j.jenvman.2016.10.064 \\
\hline $\begin{array}{c}\text { Winans K., } \\
\text { Kendall A., Deng } \\
\text { H. }\end{array}$ & $\begin{array}{l}\text { The history and current } \\
\text { applications of the circular } \\
\text { economy concept }\end{array}$ & $\begin{array}{c}\text { Renewable and } \\
\text { Sustainable Energy } \\
\text { Reviews }\end{array}$ & 2017 & 68 & & 825 & 833 & 10.1016/j.rser.2016.09.123 \\
\hline $\begin{array}{l}\text { Yap N.T., Devlin } \\
\text { J.F. }\end{array}$ & $\begin{array}{c}\text { Explaining Industrial } \\
\text { Symbiosis Emergence, } \\
\text { Development, and Disruption: } \\
\text { A Multilevel Analytical } \\
\text { Framework }\end{array}$ & $\begin{array}{l}\text { Journal of Industrial } \\
\text { Ecology }\end{array}$ & 2017 & 21 & 1 & 6 & 15 & 10.1111/jiec.12398 \\
\hline
\end{tabular}




\begin{tabular}{|c|c|c|c|c|c|c|c|c|}
\hline Author Name(s) & $\begin{array}{l}\text { Title } \\
\end{array}$ & Source Title & Year & Vol. & Issue & P. Start & P. End & Doi \\
\hline $\begin{array}{l}\text { Ohnishi S., Dong } \\
\text { H., Geng Y., Fujii } \\
\text { M., Fujita T. }\end{array}$ & $\begin{array}{c}\text { A comprehensive evaluation on } \\
\text { industrial \& urban symbiosis } \\
\text { by combining MFA, carbon } \\
\text { footprint and emergy } \\
\text { methods - Case of Kawasaki, } \\
\text { Japan } \\
\end{array}$ & Ecological Indicators & 2017 & 73 & & 315 & 324 & 10.1016/j.ecolind.2016.10.016 \\
\hline $\begin{array}{l}\text { Taddeo R., } \\
\text { Simboli A., } \\
\text { Ioppolo G., } \\
\text { Morgante A. }\end{array}$ & $\begin{array}{c}\text { Industrial symbiosis, } \\
\text { networking and innovation: } \\
\text { The potential role of innovation } \\
\text { poles }\end{array}$ & $\begin{array}{l}\text { Sustainability } \\
\text { (Switzerland) }\end{array}$ & 2017 & 9 & 2 & & & $10.3390 / \mathrm{su} 9020169$ \\
\hline $\begin{array}{l}\text { Wang Q., Deutz } \\
\text { P., Chen Y. }\end{array}$ & $\begin{array}{l}\text { Building institutional capacity } \\
\text { for industrial symbiosis } \\
\text { development: A case study of } \\
\text { an industrial symbiosis } \\
\text { coordination network in China }\end{array}$ & $\begin{array}{l}\text { Journal of Cleaner } \\
\text { Production }\end{array}$ & 2017 & 142 & & 1571 & 1582 & 10.1016/j.jclepro.2016.11.146 \\
\hline $\begin{array}{c}\text { Zhang C., } \\
\text { Romagnoli A., } \\
\text { Zhou L., Kraft M. }\end{array}$ & $\begin{array}{l}\text { Knowledge management of } \\
\text { eco-industrial park for efficient } \\
\text { energy utilization through } \\
\text { ontology-based approach }\end{array}$ & Applied Energy & 2017 & & & & & 10.1016/j.apenergy.2017.03.130 \\
\hline $\begin{array}{l}\text { Fan Y., Qiao Q., } \\
\text { Fang L., Yao Y. }\end{array}$ & $\begin{array}{c}\text { Emergy analysis on industrial } \\
\text { symbiosis of an industrial park } \\
\text { - A case study of Hefei } \\
\text { economic and technological } \\
\text { development area }\end{array}$ & $\begin{array}{l}\text { Journal of Cleaner } \\
\text { Production }\end{array}$ & 2017 & 141 & & 791 & 798 & 10.1016/j.jclepro.2016.09.159 \\
\hline $\begin{array}{c}\text { Sharib S., Halog } \\
\text { A. }\end{array}$ & $\begin{array}{l}\text { Enhancing value chains by } \\
\text { applying industrial symbiosis } \\
\text { concept to the Rubber City in } \\
\text { Kedah, Malaysia }\end{array}$ & $\begin{array}{l}\text { Journal of Cleaner } \\
\text { Production }\end{array}$ & 2017 & 141 & & 1095 & 1108 & 10.1016/j.jclepro.2016.09.089 \\
\hline $\begin{array}{l}\text { Couto Mantese } \\
\text { G., Capaldo } \\
\text { Amaral D. }\end{array}$ & $\begin{array}{l}\text { Comparison of industrial } \\
\text { symbiosis indicators through } \\
\text { agent-based modeling }\end{array}$ & $\begin{array}{l}\text { Journal of Cleaner } \\
\text { Production }\end{array}$ & 2017 & 140 & & 1652 & 1671 & 10.1016/j.jclepro.2016.09.142 \\
\hline $\begin{array}{l}\text { Fraccascia L., } \\
\text { Albino V., } \\
\text { Garavelli C.A. }\end{array}$ & $\begin{array}{l}\text { Technical efficiency measures } \\
\text { of industrial symbiosis } \\
\text { networks using enterprise } \\
\text { input-output analysis }\end{array}$ & $\begin{array}{l}\text { International Journal of } \\
\text { Production Economics }\end{array}$ & 2017 & 183 & & 273 & 286 & 10.1016/j.ijpe.2016.11.003 \\
\hline $\begin{array}{c}\text { Tseng M.-L., Bui } \\
\text { T.-D. }\end{array}$ & $\begin{array}{l}\text { Identifying eco-innovation in } \\
\text { industrial symbiosis under } \\
\text { linguistic preferences: A novel } \\
\text { hierarchical approach }\end{array}$ & $\begin{array}{l}\text { Journal of Cleaner } \\
\text { Production }\end{array}$ & 2017 & 140 & & 1376 & 1389 & 10.1016/j.jclepro.2016.10.014 \\
\hline
\end{tabular}

APPLIED ECOLOGY AND ENVIRONMENTAL RESEARCH 17(1):1159-1221.

http://www.aloki.hu • ISSN 15891623 (Print) • ISSN 17850037 (Online)

DOI: http://dx.doi.org/10.15666/aeer/1701_11591221

(c) 2019, ALÖKI Kft., Budapest, Hungary 


\begin{tabular}{|c|c|c|c|c|c|c|c|c|}
\hline Author Name(s) & Title & Source Title & Year & Vol. & Issue & P. Start & P. End & Doi \\
\hline $\begin{array}{l}\text { Kim H.-W., } \\
\text { Ohnishi S., Fujii } \\
\text { M., Fujita T., } \\
\text { Park H.-S. }\end{array}$ & $\begin{array}{l}\text { Evaluation and Allocation of } \\
\text { Greenhouse Gas Reductions in } \\
\text { Industrial Symbiosis }\end{array}$ & $\begin{array}{l}\text { Journal of Industrial } \\
\text { Ecology }\end{array}$ & 2017 & & & & & 10.1111/jiec.12539 \\
\hline $\begin{array}{c}\text { Halstenberg F.A., } \\
\text { Lindow K., Stark } \\
\text { R. }\end{array}$ & $\begin{array}{c}\text { Utilization of Product Lifecycle } \\
\text { Data from PLM Systems in } \\
\text { Platforms for Industrial } \\
\text { Symbiosis }\end{array}$ & Procedia Manufacturing & 2017 & 8 & & 369 & 376 & 10.1016/j.promfg.2017.02.047 \\
\hline $\begin{array}{l}\text { Dong L., Wang } \\
\text { Y., Scipioni A., } \\
\text { Park H.-S., Ren J. }\end{array}$ & $\begin{array}{c}\text { Recent progress on innovative } \\
\text { urban infrastructures system } \\
\text { towards sustainable resource } \\
\text { management }\end{array}$ & $\begin{array}{l}\text { Resources, } \\
\text { Conservation and } \\
\text { Recycling }\end{array}$ & 2017 & & & & & 10.1016/j.resconrec.2017.02.020 \\
\hline $\begin{array}{l}\text { Wang X., Shi } \\
\text { X.Q. }\end{array}$ & $\begin{array}{l}\text { A review of industrial ecology } \\
\text { based on GIS }\end{array}$ & $\begin{array}{l}\text { Shengtai Xuebao/ Acta } \\
\text { Ecologica Sinica }\end{array}$ & 2017 & 37 & 4 & 1346 & 1357 & $10.5846 /$ stxb201606301326 \\
\hline $\begin{array}{l}\text { Oguntoye O., } \\
\text { Evans S. }\end{array}$ & $\begin{array}{l}\text { Framing Manufacturing } \\
\text { Development in Africa and the } \\
\text { Influence of Industrial } \\
\text { Sustainability }\end{array}$ & Procedia Manufacturing & 2017 & 8 & & 75 & 80 & 10.1016/j.promfg.2017.02.009 \\
\hline $\begin{array}{l}\text { Hein A.M., } \\
\text { Yannou B., } \\
\text { Jankovic M., } \\
\text { Farel R. }\end{array}$ & $\begin{array}{c}\text { Towards an automatized } \\
\text { generation of rule-based } \\
\text { systems for architecting eco- } \\
\text { industrial parks }\end{array}$ & $\begin{array}{l}\text { Smart Innovation, } \\
\text { Systems and } \\
\text { Technologies }\end{array}$ & 2017 & 65 & & 691 & 699 & 10.1007/978-981-10-3518-0_60 \\
\hline $\begin{array}{c}\text { Dias G.M., Ayer } \\
\text { N.W., Khosla S., } \\
\text { Van Acker R., } \\
\text { Young S.B., } \\
\text { Whitney S., } \\
\text { Hendricks P. }\end{array}$ & $\begin{array}{l}\text { Life cycle perspectives on the } \\
\text { sustainability of Ontario } \\
\text { greenhouse tomato production: } \\
\text { Benchmarking and } \\
\text { improvement opportunities }\end{array}$ & $\begin{array}{l}\text { Journal of Cleaner } \\
\text { Production }\end{array}$ & 2017 & 140 & & 831 & 839 & 10.1016/j.jclepro.2016.06.039 \\
\hline $\begin{array}{l}\text { Medina-González } \\
\text { S., Graells M., } \\
\text { Guillén-Gosálbez } \\
\text { G., Espuña A., } \\
\text { Puigjaner L. } \\
\end{array}$ & $\begin{array}{l}\text { Systematic approach for the } \\
\text { design of sustainable supply } \\
\text { chains under quality } \\
\text { uncertainty }\end{array}$ & $\begin{array}{l}\text { Energy Conversion and } \\
\text { Management }\end{array}$ & 2017 & & & & & 10.1016/j.enconman.2017.02.060 \\
\hline $\begin{array}{l}\text { Malinauskiene } \\
\text { M., Kliopova I., } \\
\text { Hugi C., Staniškis } \\
\text { J.K. }\end{array}$ & $\begin{array}{l}\text { Geostrategic Supply Risk and } \\
\text { Economic Importance as } \\
\text { Drivers for Implementation of } \\
\text { Industrial Ecology Measures in } \\
\text { a Nitrogen Fertilizer } \\
\text { Production Company }\end{array}$ & $\begin{array}{c}\text { Journal of Industrial } \\
\text { Ecology }\end{array}$ & 2017 & & & & & 10.1111/jiec.12561 \\
\hline
\end{tabular}




\begin{tabular}{c|c|c|c|c|c|c|c|c}
\hline Author Name(s) & Title & Source Title & Year & Vol. & Issue & P. Start & P. End & \\
\hline $\begin{array}{c}\text { Deutz P., Baxter } \\
\text { H., Gibbs D., } \\
\text { Mayes W.M., } \\
\text { Gomes H.I. }\end{array}$ & $\begin{array}{c}\text { Resource recovery and } \\
\text { remediation of highly alkaline } \\
\text { residues: A political-industrial }\end{array}$ & $\begin{array}{c}\text { Geoforum } \\
\text { circulac economy }\end{array}$ & 2017 & & & & \\
\hline
\end{tabular}

Appendix-II. Top ten countries in IS research with industrial growth indicators

\begin{tabular}{|c|c|c|c|}
\hline Countries & No. of Papers & Annual Industrial Growth rate & Industrial production in Price (million US\$) \\
\hline China & 85 & $6.10 \%$ & 474,000 \\
\hline USA & 49 & $0.10 \%$ & 351,600 \\
\hline UK & 49 & $3.10 \%$ & 38,500 \\
\hline Italy & 34 & $1.80 \%$ & 39,400 \\
\hline Japan & 27 & $2.00 \%$ & 138,000 \\
\hline Netherland & 21 & $3.10 \%$ & 13,100 \\
\hline Philippines & 21 & $7.20 \%$ & 9,920 \\
\hline Australia & 19 & $-1.60 \%$ & 369,400 \\
\hline Canada & 19 & $4.80 \%$ & 22,200 \\
\hline France & 19 & $1.30 \%$ & 30,900 \\
\hline
\end{tabular}

